\title{
Thiourea-Catalyzed Enantioselective Hydrophosphonylation of Imines: Practical Access to Enantiomerically Enriched $\alpha$-Amino Phosphonic Acids
}

\author{
Guy D. Joly and Eric N. Jacobsen* \\ Department of Chemistry and Chemical Biology, Harvard University, 12 Oxford Street, Cambridge, \\ Massachusetts 02138
}

\section{Supporting Information}

General Procedures. All hydrophosphonylation reactions were performed in oven dried 4-mL to $15-\mathrm{mL}$ vials using unpurified commercial diethyl ether. The vials were sealed with plastic screw caps, and the reactions were run under an ambient atmosphere. All other reactions were performed under an atmosphere of nitrogen in oven-dried or flame-dried round bottomed flasks fitted with rubber septa and/or glass stoppers. Stainless steel syringes or cannulae were used to transfer moisture sensitive liquids.

Materials. Commercial reagents were used as received with the following exceptions: dichloromethane was distilled from calcium hydride at 760 Torr, and toluene was distilled from sodium at 760 Torr. Flash chromatography was performed using silica gel 60 (230-400 mesh), purchased from EM Science. Catalysts $\mathbf{1 \mathbf { a } ^ { 1 }}$ and $\mathbf{1} \mathbf{b}^{2}$ were prepared as previously described.

Instrumentation. Proton nuclear magnetic resonance $\left({ }^{1} \mathrm{H}\right.$ NMR) spectra, carbon nuclear magnetic resonance $\left({ }^{13} \mathrm{C}\right.$ NMR) spectra, and phosphorus nuclear magnetic resonance $\left({ }^{31} \mathrm{P}\right.$ NMR) spectra were recorded on Varian-Mercury-400 (400 MHz) or an Inova-500 (500 MHz). Chemical shifts for protons are reported in parts per million downfield from tetramethylsilane and are referenced to residual protium in the NMR solvent $\left(\mathrm{CHCl}_{3}: \delta 7.26 ; \mathrm{H}_{2} \mathrm{O}: \delta 4.79\right)$. Chemical shifts for carbon are reported in parts per million downfield from tetramethylsilane and are referenced to the carbon resonances of the solvent $\left(\mathrm{CDCl}_{3}: \delta\right.$ 77.16) or to dioxane as an internal standard in $\mathrm{D}_{2} \mathrm{O}$ (dioxane: $\delta$ 77.16). Chemical shifts for phosphorus are reported in parts per million downfield from phosphoric acid $\left(\mathrm{H}_{3} \mathrm{PO}_{4}\right)$ and are referenced to $85 \% \mathrm{H}_{3} \mathrm{PO}_{4}$ as an external standard. All phosphorus nuclear magnetic resonance spectra are proton-decoupled unless indicated otherwise. Data are presented as follows: chemical shift, integration, multiplicity $(\mathrm{br}=$ broad, $\mathrm{s}=$ singlet, $\mathrm{d}=$ doublet, $\mathrm{t}=$ triplet, $\mathrm{q}=$ quartet, $\mathrm{m}=$ multiplet $)$, coupling constants in Hertz $(\mathrm{Hz})$, and assignment. Infrared (IR) spectra were obtained on a Mattson Galaxy Series FTIR 3000 spectrophotometer referenced to a polystyrene standard. Data are presented as follows: frequency of absorption $\left(\mathrm{cm}^{-1}\right)$ and intensity of absorption $(\mathrm{s}=$ strong, $\mathrm{m}=$ medium, $\mathrm{w}=$ weak). Optical rotations were measured using a $2 \mathrm{~mL}$ cell with a $1 \mathrm{dm}$ path length on a Jasco DIP 370 digital polarimeter. Melting points were measured on a Mel-Temp apparatus and are uncorrected. The mass spectroscopic data were obtained at the Harvard University mass spectrometry facility. Chiral HPLC analysis was performed on a Shimadzu VP-series instrument. 


\section{Catalyst Optimization.}

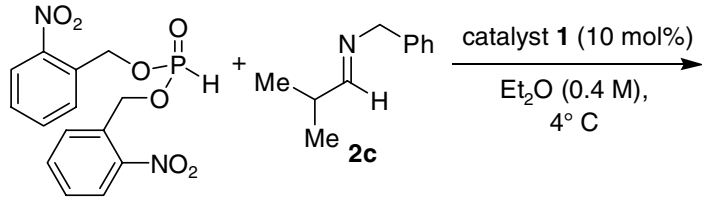

$3 \mathrm{~h}$

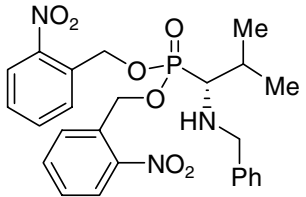

$4 \mathrm{c}$

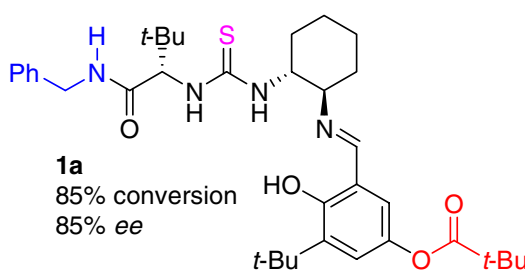<smiles>CC(C)(C)c1cc(/C=N/[C@H]2CCCC[C@H]2NC(=S)NC(C(=O)O[13CH3])C(=O)O[13CH3])c(O)c(C(C)(C)C)c1</smiles><smiles>C=NC1CCCC[C@H]1NC(=O)N[C@H](C(=O)NCc1ccccc1)C(C)(C)C</smiles>

$1 \mathrm{i}$ $92 \%$ conversion $84 \%$ ee
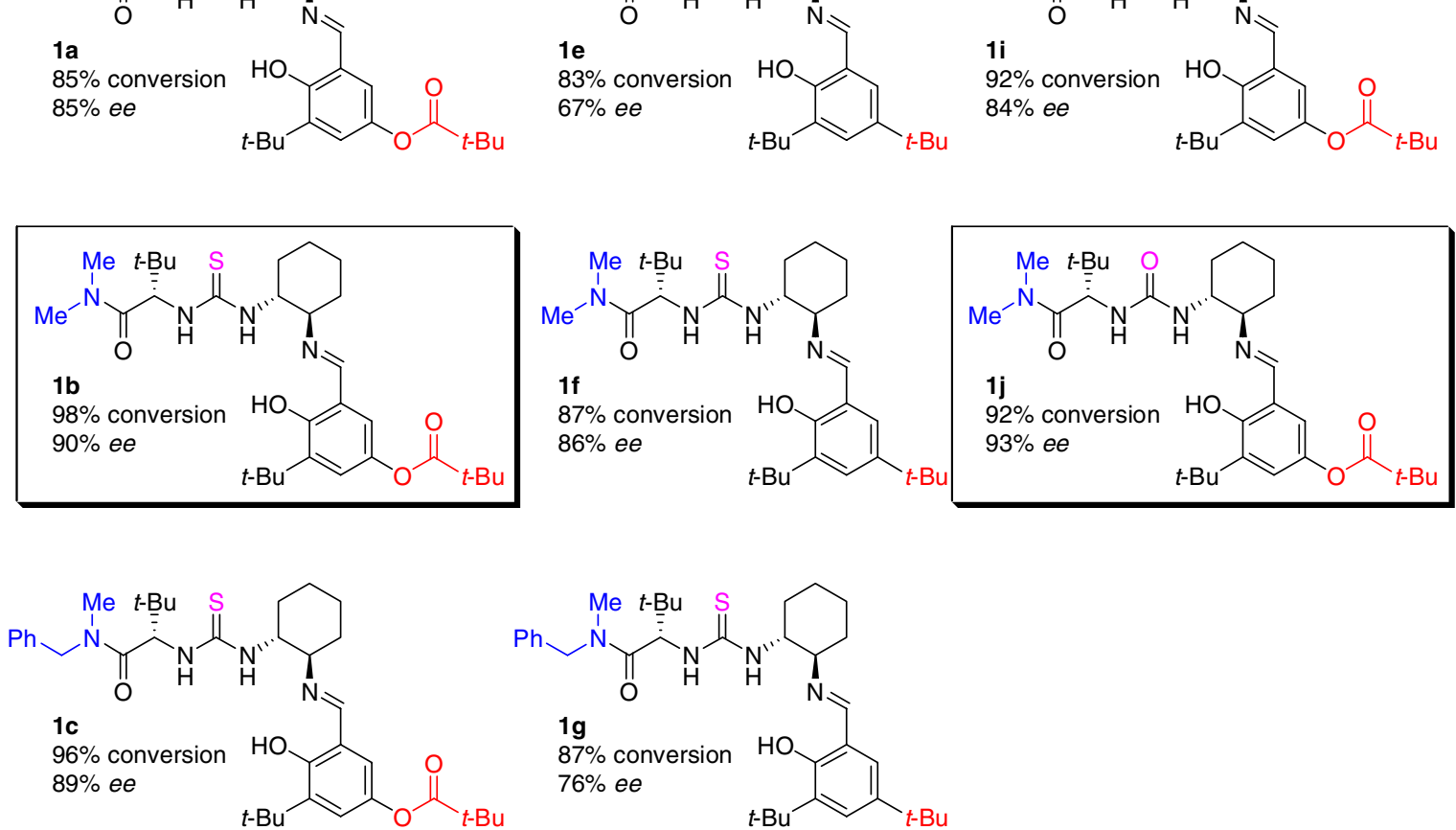<smiles>C=NC1CCCC[C@H]1NC(=S)N[C@@H](C(=O)N(Cc1ccccc1)Cc1ccccc1)C(C)(C)C</smiles>

1d

$95 \%$ conversion $89 \%$ ee<smiles>CC(C)(C)C(=O)Oc1cc(C=N)c(O)c(C(C)(C)C)c1</smiles><smiles>CC(C)(C)[C@H](NC=S)C(=O)N(Cc1ccccc1)Cc1ccccc1</smiles><smiles>CC(C)(C)c1cc(/C=N/C2CCCC[C@H]2N)c(O)c(C(C)(C)C)c1</smiles>

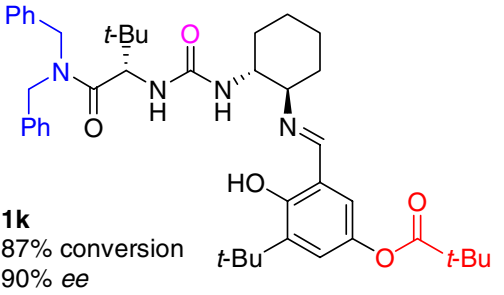

Note: $\%$ conversion and $\%$ ee determined after $24 \mathrm{~h} . \%$ Conversion determined by ${ }^{1} \mathrm{H}-\mathrm{NMR}$.

Catalysts $\mathbf{1 b}$ and $\mathbf{1 j}$ were selected for further comparison. 


\section{Comparison of Catalysts $\mathbf{1 b}$ and $\mathbf{1 j}$.}<smiles>[CH2+]P(=O)(O)OCc1ccccc1[N+](=O)[O-]</smiles>

$3 \mathrm{~h}$

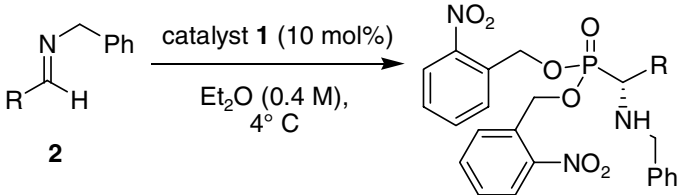

4<smiles>CN(C)C(=O)C(NC(=S)N[C@H]1CCCC[C@@H]1N=Cc1cc(OC(=O)C(C)(C)C)cc(C(C)(C)C)c1O)C(C)(C)C</smiles><smiles>CN(C)C(=O)C(NC(=O)N[C@H]1CCCC[C@H]1N=Cc1cc(OC(=O)C(C)(C)C)cc(C(C)(C)C)c1O)C(C)(C)C</smiles>

\begin{tabular}{c|cccc}
\hline substrate & catalyst & time $(\mathrm{h})$ & \% conv & \%ee \\
\hline & thiourea 1b & 48 & 87 & 98 \\
\hline & urea 1j & 77 & 81 & 96 \\
\hline
\end{tabular}

\begin{tabular}{ccccc} 
thiourea $\mathbf{1 b}$ & 48 & 86 & 98 \\
urea $\mathbf{1 j}$ & 77 & 74 & 97 \\
\hline thiourea $\mathbf{1 b}$ & 24 & 95 & 96 \\
\hline urea $\mathbf{1 j}$ & 30 & 69 & 92 \\
\hline
\end{tabular}

${ }^{a}$ Determined by ${ }^{1} \mathrm{H}-\mathrm{NMR}$.

Thiourea catalyst $\mathbf{1 b}$ is generally superior to urea catalyst $\mathbf{1} \mathbf{j}$ in terms of both reaction rate and enantioselectivity. Hydrophosphonylation of imine $\mathbf{2 c}$ provides the lone exception. Thiourea $\mathbf{1 b}$ was selected as the optimal catalyst and was employed in the exploration of additional imine substrates 2 (see Table 2). 


\section{Imine Preparation. ${ }^{3}$}
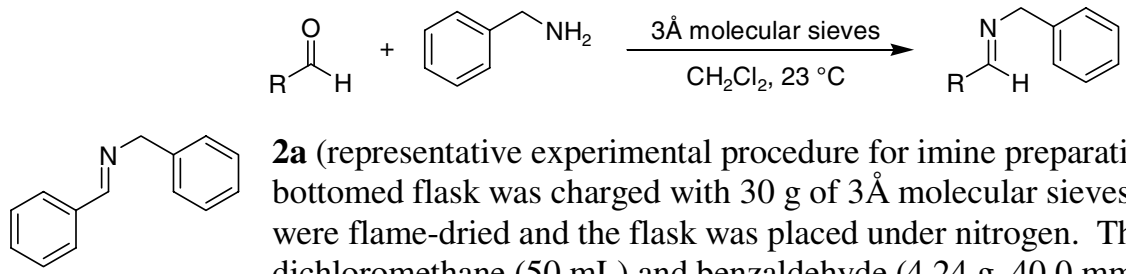

2a (representative experimental procedure for imine preparation): A 250-mL round bottomed flask was charged with $30 \mathrm{~g}$ of $3 \AA$ molecular sieves. The molecular sieves were flame-dried and the flask was placed under nitrogen. The flask was charged with dichloromethane $(50 \mathrm{~mL})$ and benzaldehyde $(4.24 \mathrm{~g}, 40.0 \mathrm{mmol}, 1.02$ equiv).

Benzylamine ( $4.20 \mathrm{~g}, 39.2 \mathrm{mmol}, 1.0$ equiv) was added slowly over two minutes. The reaction was monitored by ${ }^{1} \mathrm{H}$ NMR. After $3 \mathrm{~h}$, the reaction was vacuum filtered to remove the molecular sieves and was concentrated in vacuo. The crude oil was purified by vacuum distillation $\left(118^{\circ} \mathrm{C}, 0.05 \mathrm{~mm} \mathrm{Hg}\right)$ to provide $2 \mathbf{a}(5.82 \mathrm{~g}, 29.8 \mathrm{mmol}, 76 \%)$ as a colorless liquid. IR (thin film, $\left.\mathrm{cm}^{-1}\right) 3084(\mathrm{w}), 3063(\mathrm{~m}), 3028$ (m), 2870 (m), 2837 (m), 1643 (s), 1495 (m), 1452 (s), 1312 (m), 1026 (m); ${ }^{1} \mathrm{H}$ NMR (500 MHz, $\left.\mathrm{CDCl}_{3}\right) \delta$ $8.41(1 \mathrm{H}, \mathrm{s}, \mathrm{CH}=\mathrm{N}), 7.81-7.78(2 \mathrm{H}, \mathrm{m}, \mathrm{ArH}), 7.44-7.40(3 \mathrm{H}, \mathrm{m}, \mathrm{ArH}), 7.37-7.33$ (4H, m, ArH), 7.29-7.25 $(1 \mathrm{H}, \mathrm{m}, \mathrm{ArH}), 4.84\left(2 \mathrm{H}, \mathrm{d}, J=1.5 \mathrm{~Hz}, \mathrm{CH}_{2}-\mathrm{N}\right) ;{ }^{13} \mathrm{C} \mathrm{NMR}\left(101 \mathrm{MHz}, \mathrm{CDCl}_{3}\right) \delta 161.8,139.3,136.1,130.7$, 128.5, 128.5, 128.2, 127.9, 126.9, 65.0; HRMS (EI) $\mathrm{m} / \mathrm{z}$ for $\mathrm{C}_{14} \mathrm{H}_{13} \mathrm{~N}[\mathrm{M}]^{+}$calcd. 195.1048, found 195.1048 .

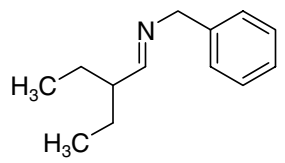

2b: Reaction was performed on a $40 \mathrm{mmol}$ scale using 2-ethylbutyraldehyde ( $4.84 \mathrm{~g}$, $48.3 \mathrm{mmol})$ and benzylamine $(4.32 \mathrm{~g}, 40.3 \mathrm{mmol})$. The product was purified by vacuum distillation $\left(80^{\circ} \mathrm{C}, 0.05 \mathrm{~mm} \mathrm{Hg}\right)$ to provide $2 \mathbf{b}(5.72 \mathrm{~g}, 30.2 \mathrm{mmol}, 75 \%)$ as a colorless liquid. IR (thin film, $\mathrm{cm}^{-1}$ ) 3063 (w), 3030 (m), 2963 (s), 2930 (s), 2876 (s), 1667 (s), 1495 (m), 1454 (s), 1377 (m), 1028 (m); ${ }^{1} \mathrm{H}$ NMR (500 MHz, CDCl $\left.)_{3}\right) \quad 7.55(1 \mathrm{H}, \mathrm{d}, J=6.8 \mathrm{~Hz}$, $\mathrm{CH}=\mathrm{N}), 7.35-7.30(2 \mathrm{H}, \mathrm{m}, \mathrm{ArH}), 7.27-7.22(3 \mathrm{H}, \mathrm{m}, \mathrm{ArH}), 4.59\left(2 \mathrm{H}, \mathrm{s}, \mathrm{CH}_{2}-\mathrm{N}\right), 2.17-2.09(1 \mathrm{H}, \mathrm{m}$, $\left.\mathrm{CH}\left(\mathrm{CH}_{2} \mathrm{CH}_{3}\right)_{2}\right), 1.57-1.44\left(4 \mathrm{H}, \mathrm{m}, \mathrm{CH}_{2} \mathrm{CH}_{3}\right), 0.91\left(6 \mathrm{H}, \mathrm{t}, J=7.3 \mathrm{~Hz}, \mathrm{CH}_{3}\right) ;{ }^{13} \mathrm{C}$ NMR $\left(101 \mathrm{MHz}, \mathrm{CDCl}_{3}\right) \delta$ 170.2, 139.3, 128.3, 127.7, 126.7, 64.8, 48.1, 25.0, 11.6; HRMS (ES) $\mathrm{m} / \mathrm{z}$ for $\mathrm{C}_{13} \mathrm{H}_{20} \mathrm{~N}[\mathrm{M}+\mathrm{H}]^{+}$calcd. 190.1595 , found 190.1600 .<smiles>CC(C)/C=N/Cc1ccccc1</smiles>

2c: Reaction was performed on a $40 \mathrm{mmol}$ scale using isobutyraldehyde $(5.77 \mathrm{~g}, 80$ $\mathrm{mmol}$ ) and benzylamine ( $4.32 \mathrm{~g}, 40.3 \mathrm{mmol})$. The product was purified by vacuum distillation $\left(123^{\circ} \mathrm{C}, 30 \mathrm{~mm} \mathrm{Hg}\right)$ to provide $2 \mathrm{c}(4.42 \mathrm{~g}, 27.4 \mathrm{mmol}, 68 \%)$ as a colorless liquid. IR (thin film, $\mathrm{cm}^{-1}$ ) 3030 (m), 2965 (s), 2874 (s), $1670(\mathrm{~s}), 1495(\mathrm{~m}), 1454$ (s), $1368(\mathrm{~m}), 1030(\mathrm{w}) ;{ }^{1} \mathrm{H}$ NMR $\left(500 \mathrm{MHz}, \mathrm{CDCl}_{3}\right) \delta$ 7.68-7.65 (1H, m, CH=N), 7.35-7.30 (2H, m, ArH), 7.27-7.22 (3H, m, ArH), $4.56\left(2 \mathrm{H}, \mathrm{s}, \mathrm{CH}_{2}-\mathrm{N}\right) 2.56-2.46\left(1 \mathrm{H}, \mathrm{m}, \mathrm{CH}\left(\mathrm{CH}_{3}\right)_{2}\right), 1.12\left(6 \mathrm{H}, \mathrm{d}, J=6.8 \mathrm{~Hz}, \mathrm{CH}_{3}\right)$; ${ }^{13} \mathrm{C}$ NMR $\left(101 \mathrm{MHz}, \mathrm{CDCl}_{3}\right) \delta 171.0,139.5,128.4,127.8,126.9,64.9,34.2,19.4 ;$ LRMS (CI) m/z: 161.8 $(100 \%)[\mathrm{M}+\mathrm{H}]^{+}$.

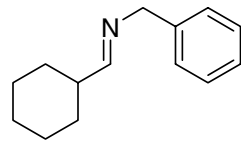

2d: Reaction was performed on a $38 \mathrm{mmol}$ scale using cyclohexancarboxaldehyde (4.44 $\mathrm{g}, 39.6 \mathrm{mmol})$ and benzylamine $(4.04 \mathrm{~g}, 37.7 \mathrm{mmol})$. The product was purified by vacuum distillation $\left(111^{\circ} \mathrm{C}, 0.05 \mathrm{~mm} \mathrm{Hg}\right)$ to provide $2 \mathrm{~d}(5.86 \mathrm{~g}, 29.1 \mathrm{mmol}, 77 \%)$ as a colorless liquid. IR (thin film, $\mathrm{cm}^{-1}$ ) 3044 (w), 2990 (m), 2926 (m), 1667 (s), 1495 (s), 1451 (s), 1343 (m), 1028 (m), $966(\mathrm{~m}) ;{ }^{1} \mathrm{H}$ NMR $\left(500 \mathrm{MHz}, \mathrm{CDCl}_{3}\right) \delta 7.64(1 \mathrm{H}, \mathrm{d}, J=4.9 \mathrm{~Hz}, \mathrm{CH}=\mathrm{N})$, 7.34-7.30 (2H, m, ArH), 7.26-7.22 (3H, m, ArH), $4.55\left(2 \mathrm{H}, \mathrm{s}, \mathrm{CH}_{2}-\mathrm{N}\right), 2.29-2.20(1 \mathrm{H}, \mathrm{m}, c$-hexH), 1.88$1.64\left(5 \mathrm{H}, c\right.$-hexH), 1.36-1.16 (5H, m, $c$-hexH); $\left.{ }^{13} \mathrm{C} \mathrm{NMR} \mathrm{(101} \mathrm{MHz,} \mathrm{CDCl}_{3}\right) \delta 170.2,139.6,128.4,127.8$, 126.8, 65.0, 43.6, 29.7, 26.0, 25.5; LRMS (CI) m/z: $201.8(100 \%)[\mathrm{M}+\mathrm{H}]^{+}$.

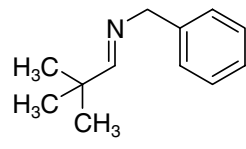

2e: Reaction was performed on a $40 \mathrm{mmol}$ scale using trimethylacetaldehyde $(3.81 \mathrm{~g}$, $44.2 \mathrm{mmol})$ and benzylamine $(4.32 \mathrm{~g}, 40.3 \mathrm{mmol})$. The product was purified by vacuum distillation $\left(129^{\circ} \mathrm{C}, 30 \mathrm{~mm} \mathrm{Hg}\right)$ to provide $2 \mathrm{e}(4.87 \mathrm{~g}, 27.8 \mathrm{mmol}, 69 \%)$ as a colorless liquid. IR (thin film, cm $\left.{ }^{-1}\right) 2959$ (s), 2812 (s), 1667 (s), 1497 (m), 1476 (m), 1454 (s), 1366 (s), 1208 (w), 1051 (w), $1030(\mathrm{w}) ;{ }^{1} \mathrm{H}$ NMR $\left(500 \mathrm{MHz}, \mathrm{CDCl}_{3}\right) \delta 7.66(1 \mathrm{H}, \mathrm{s}, \mathrm{CH}=\mathrm{N}), 7.34-7.30(2 \mathrm{H}$, $\mathrm{m}, \operatorname{ArH}), 7.27-7.22(3 \mathrm{H}, \mathrm{m}, \mathrm{ArH}), 4.58\left(2 \mathrm{H}, \mathrm{s}, \mathrm{CH}_{2}-\mathrm{N}\right), 1.12\left(9 \mathrm{H}, \mathrm{s},\left(\mathrm{CH}_{3}\right)_{3}\right) ;{ }^{13} \mathrm{C} \mathrm{NMR}\left(101 \mathrm{MHz}, \mathrm{CDCl}_{3}\right)$ 
$\delta$ 173.5, 139.8, 128.4, 127.6, 126.8, 64.6, 36.4, 27.0; HRMS (CI) m/z for $\mathrm{C}_{12} \mathrm{H}_{18} \mathrm{~N}[\mathrm{M}+\mathrm{H}]^{+}$calcd. 176.1439, found 176.1440 .<smiles>CC(C)=C/C=C\NCc1ccccc1</smiles>

2f: Reaction was performed on a $40 \mathrm{mmol}$ scale using 3-methyl-2-butenal (4.01 g, $47.7 \mathrm{mmol}$ ) and benzylamine $(4.32 \mathrm{~g}, 40.3 \mathrm{mmol})$. The product was purified by vacuum distillation $\left(112^{\circ} \mathrm{C}, 0.05 \mathrm{~mm} \mathrm{Hg}\right)$ to provide $2 \mathbf{f}(4.33 \mathrm{~g}, 25.0 \mathrm{mmol}, 62 \%)$ as a red-orange liquid. IR (thin film, $\mathrm{cm}^{-1}$ ) $3028(\mathrm{~m}), 2864$ (s), 1651 (s), 1451 (s), 1377 (s), $1204(\mathrm{~m}), 855$ (m), $733(\mathrm{~s}) ;{ }^{1} \mathrm{H}$ NMR $\left(500 \mathrm{MHz}, \mathrm{CDCl}_{3}\right) \delta 8.28(1 \mathrm{H}, \mathrm{d}, J=9.3 \mathrm{~Hz}, \mathrm{CH}=\mathrm{N}), 7.35-7.22(5 \mathrm{H}, \mathrm{m}, \mathrm{ArH}), 6.09-6.06$ $(1 \mathrm{H}, \mathrm{m}, \mathrm{CH}=\mathrm{C}), 4.65\left(2 \mathrm{H}, \mathrm{s}, \mathrm{CH}_{2}-\mathrm{N}\right), 1.95\left(3 \mathrm{H}, \mathrm{s}, \mathrm{CH}_{3}\right), 1.89\left(3 \mathrm{H}, \mathrm{s}, \mathrm{CH}_{3}\right) ;{ }^{13} \mathrm{C} \mathrm{NMR}\left(101 \mathrm{MHz}, \mathrm{CDCl}_{3}\right) \delta$ 160.0, 146.7, 139.4, 128.2, 127.7, 126.6, 125.3, 65.1, 26.3, 18.4; HRMS (EI) $\mathrm{m} / \mathrm{z}$ for $\mathrm{C}_{12} \mathrm{H}_{15} \mathrm{~N}[\mathrm{M}]^{+}$calcd. 173.1204, found 173.1204.

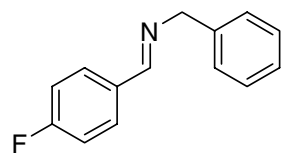

2g: Reaction was performed on a $39 \mathrm{mmol}$ scale using 4-fluorobenzaldehyde (4.98 g, $40.1 \mathrm{mmol})$ and benzylamine $(4.22 \mathrm{~g}, 39.4 \mathrm{mmol})$. The product was purified by vacuum distillation $\left(120^{\circ} \mathrm{C}, 0.05 \mathrm{~mm} \mathrm{Hg}\right)$ to provide $2 \mathrm{~g}(5.97 \mathrm{~g}, 28.0 \mathrm{mmol}, 71 \%)$ as a pale yellow liquid. IR (thin film, $\left.\mathrm{cm}^{-1}\right) 3063(\mathrm{~m}), 3030(\mathrm{~m}), 2841(\mathrm{~m}), 1643(\mathrm{~s})$,

1601 (s), 1508 (s), 1231 (s), $1152(\mathrm{~m}), 1028(\mathrm{~m}), 837$ (s); ${ }^{1} \mathrm{H}$ NMR $\left(500 \mathrm{MHz}, \mathrm{CDCl}_{3}\right) \delta 8.36(1 \mathrm{H}, \mathrm{s}$, $\mathrm{CH}=\mathrm{N}), 7.81-7.76(2 \mathrm{H}, \mathrm{m}, \operatorname{ArH}), 7.38-7.26(5 \mathrm{H}, \mathrm{m}, \mathrm{ArH}), 7.14-7.08(2 \mathrm{H}, \mathrm{m}, \operatorname{ArH}), 4.82\left(2 \mathrm{H}, \mathrm{s}, \mathrm{CH}_{2}-\mathrm{N}\right)$; ${ }^{13} \mathrm{C}$ NMR $\left(101 \mathrm{MHz}, \mathrm{CDCl}_{3}\right) \delta 164.2(\mathrm{~d}, J=251 \mathrm{~Hz}), 160.2(\mathrm{~s}), 139.2(\mathrm{~s}), 132.4(\mathrm{~d}, J=2.8 \mathrm{~Hz}), 130.1(\mathrm{~d}, J$ $=8.7 \mathrm{~Hz}), 128.4(\mathrm{~s}), 127.9(\mathrm{~s}), 126.9(\mathrm{~s}), 115.5(\mathrm{~d}, J=22.0 \mathrm{~Hz}), 64.8(\mathrm{~s}) ; \mathrm{HRMS}(\mathrm{EI}) \mathrm{m} / \mathrm{z}_{\text {for }} \mathrm{C}_{14} \mathrm{H}_{12} \mathrm{FN}$ $[\mathrm{M}]^{+}$calcd. 213.0954, found 213.0956.

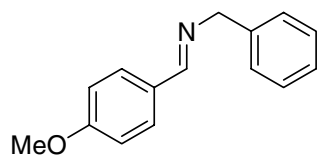

2h: Reaction was performed on a 39 mmol scale using 4-methoxybenzaldehyde $(5.60 \mathrm{~g}, 41.1 \mathrm{mmol})$ and benzylamine $(4.19 \mathrm{~g}, 39.1 \mathrm{mmol})$. The product was purified by vacuum distillation $\left(145^{\circ} \mathrm{C}, 0.05 \mathrm{~mm} \mathrm{Hg}\right)$ to provide $2 \mathbf{h}(6.93 \mathrm{~g}, 30.8$ $\mathrm{mmol}, 75 \%)$ as a colorless liquid which slowly solidified to a white crystalline solid. $\mathrm{mp}=39-40^{\circ} \mathrm{C}$; IR (thin film, $\mathrm{cm}^{-1}$ ) $3028(\mathrm{w}), 2837(\mathrm{~m}), 1647$ (s), $1606(\mathrm{~s}), 1512(\mathrm{~s}), 1452(\mathrm{~m}), 1305$ (m), $1250(\mathrm{~s}), 1029(\mathrm{~m}), 832(\mathrm{~m}) ;{ }^{1} \mathrm{H}$ NMR $\left(500 \mathrm{MHz}, \mathrm{CDCl}_{3}\right) \delta 8.33(1 \mathrm{H}, \mathrm{s}, \mathrm{CH}=\mathrm{N}), 7.73(2 \mathrm{H}, \mathrm{d}, J=8.8$ $\mathrm{Hz}, \mathrm{ArH}), 7.37-7.33(3 \mathrm{H}, \mathrm{m}, \mathrm{ArH}), 7.29-7.24(2 \mathrm{H}, \mathrm{m}, \mathrm{ArH}), 6.93(2 \mathrm{H}, \mathrm{d}, J=8.8 \mathrm{~Hz}, \mathrm{ArH}), 4.79$ (2H, s, $\left.\mathrm{CH}_{2}-\mathrm{N}\right), 3.85\left(3 \mathrm{H}, \mathrm{s}, \mathrm{OCH}_{3}\right) ;{ }^{13} \mathrm{C}$ NMR $\left(101 \mathrm{MHz}, \mathrm{CDCl}_{3}\right) \delta 161.6,161.1,139.6,129.7,129.1,128.4$, 127.9, 126.8, 113.9, 64.8, 55.1; HRMS (ES) $\mathrm{m} / \mathrm{z}$ for $\mathrm{C}_{15} \mathrm{H}_{16} \mathrm{NO}[\mathrm{M}+\mathrm{H}]^{+}$calcd. 226.1232, found 226.1225.

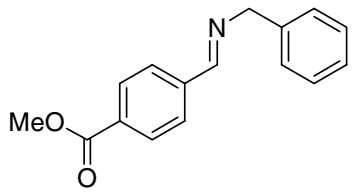

2i: Reaction was performed on a $36 \mathrm{mmol}$ scale using methyl 4-formylbenzoate $(6.00 \mathrm{~g}, 36.5 \mathrm{mmol})$ and benzylamine $(3.92 \mathrm{~g}, 36.6 \mathrm{mmol})$. The product was purified by recrystallization from hexanes to provide $2 \mathbf{i}(5.06 \mathrm{~g}, 20.0 \mathrm{mmol}$, $55 \%$ ) as a white crystalline solid. $\mathrm{mp}=91-94^{\circ} \mathrm{C}$; IR (thin film, $\left.\mathrm{cm}^{-1}\right) 3082(\mathrm{w})$, $2953(\mathrm{w}), 2859(\mathrm{w}), 1711(\mathrm{~s}), 1643(\mathrm{~s}), 1433(\mathrm{~m}), 1373(\mathrm{~m}), 1279(\mathrm{~s}), 1113(\mathrm{~s})$

$1045(\mathrm{~m}), 735(\mathrm{~s}) ;{ }^{1} \mathrm{H}$ NMR $\left(500 \mathrm{MHz}, \mathrm{CDCl}_{3}\right) \delta 8.44(1 \mathrm{H}, \mathrm{s}, \mathrm{CH}=\mathrm{N}), 8.08(2 \mathrm{H}, \mathrm{d}, J=8.1 \mathrm{~Hz}, \mathrm{ArH}), 7.85$ $(2 \mathrm{H}, \mathrm{d}, J=8.4 \mathrm{~Hz}, \operatorname{ArH}), 7.39-7.26(5 \mathrm{H}, \mathrm{m}, \operatorname{ArH}), 4.86\left(2 \mathrm{H}, \mathrm{d}, J=1.5 \mathrm{~Hz}, \mathrm{CH}_{2}-\mathrm{N}\right), 3.93\left(3 \mathrm{H}, \mathrm{s}, \mathrm{OCH}_{3}\right)$; ${ }^{13} \mathrm{C}$ NMR $\left(101 \mathrm{MHz}, \mathrm{CDCl}_{3}\right) \delta 166.6,160.9,140.1,138.9,131.9,129.9,128.6,128.2,128.1,127.2,65.2$, 52.3; LRMS (CI) m/z: $253.7(100 \%)[\mathrm{M}+\mathrm{H}]^{+}$.

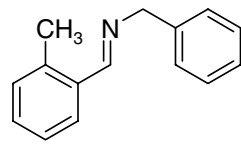

2j: Reaction was performed on a $40 \mathrm{mmol}$ scale using 2-methylbenzaldehyde (5.07 g, $42.2 \mathrm{mmol}$ ) and benzylamine $(4.32 \mathrm{~g}, 40.3 \mathrm{mmol})$. The product was purified by vacuum distillation $\left(130^{\circ} \mathrm{C}, 0.05 \mathrm{~mm} \mathrm{Hg}\right)$ to provide $\mathbf{2 j}(6.45 \mathrm{~g}, 30.8 \mathrm{mmol}, 76 \%)$ as a colorless liquid. IR (thin film, $\mathrm{cm}^{-1}$ ) 3063 (s), 3028 (s), 2882 (s), 1638 (s), 1603 (s), 1495 (s), 1452 (s), 1289 (s), 1028 (s), 698 (s); ${ }^{1} \mathrm{H}$ NMR (500 MHz, $\left.\mathrm{CDCl}_{3}\right) \delta 8.72(1 \mathrm{H}, \mathrm{s}, \mathrm{CH}=\mathrm{N}), 7.94(1 \mathrm{H}, \mathrm{dd}, J=$ 7.8, $1.5 \mathrm{~Hz}, \mathrm{ArH}), 7.39-7.18(8 \mathrm{H}, \mathrm{m}, \mathrm{ArH}), 4.86\left(2 \mathrm{H}, \mathrm{d}, J=1.5 \mathrm{~Hz}, \mathrm{CH}_{2}-\mathrm{N}\right), 2.54\left(3 \mathrm{H}, \mathrm{s}, \mathrm{ArCH}_{3}\right) ;{ }^{13} \mathrm{C}$ NMR $\left(101 \mathrm{MHz}, \mathrm{CDCl}_{3}\right) \delta 160.6,139.6,137.7,134.2,130.8,130.3,128.5,127.9,127.8,126.9,126.2$, 65.6, 19.4; LRMS (CI) m/z: $209.7(100 \%)[\mathrm{M}+\mathrm{H}]^{+}$.

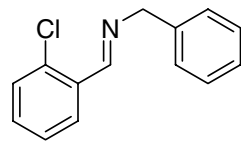

2k: Reaction was performed on a $41 \mathrm{mmol}$ scale using 2-chlorobenzaldehyde $(5.99 \mathrm{~g}$, $42.6 \mathrm{mmol})$ and benzylamine $(4.35 \mathrm{~g}, 40.6 \mathrm{mmol})$. The product was purified by vacuum distillation $\left(136^{\circ} \mathrm{C}, 0.05 \mathrm{~mm} \mathrm{Hg}\right)$ to provide $2 \mathbf{k}(6.27 \mathrm{~g}, 27.3 \mathrm{mmol}, 67 \%)$ as a colorless 
liquid. IR (thin film, $\mathrm{cm}^{-1}$ ) 3029 (s), 2890 (s), 1634 (s), 1593 (s), 1444 (s), 1373 (s), 1272 (s), 1052 (s), $1028(\mathrm{~s}) ;{ }^{1} \mathrm{H}$ NMR $\left(500 \mathrm{MHz}, \mathrm{CDCl}_{3}\right) \delta 8.86(1 \mathrm{H}, \mathrm{s}, \mathrm{CH}=\mathrm{N}), 8.10(1 \mathrm{H}, \mathrm{dd}, J=7.3,1.5 \mathrm{~Hz}, \mathrm{ArH}), 7.40-7.26$ $(8 \mathrm{H}, \mathrm{m}, \mathrm{ArH}), 4.87\left(2 \mathrm{H}, \mathrm{d}, J=1.0 \mathrm{~Hz}, \mathrm{CH}_{2}-\mathrm{N}\right) ;{ }^{13} \mathrm{C} \mathrm{NMR}\left(101 \mathrm{MHz}, \mathrm{CDCl}_{3}\right) \delta 158.4,139.0,135.1,133.1$, 131.5, 129.6, 128.4 128.4, 127.9, 127.0, 126.9, 65.2; HRMS (ES) m/z for $\mathrm{C}_{14} \mathrm{H}_{13} \mathrm{CIN}[\mathrm{M}+\mathrm{H}]^{+}$calcd. 230.0736 , found 230.0731 .

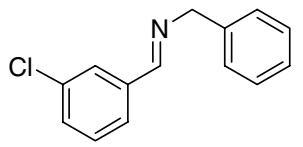

2I: Reaction was performed on a $40 \mathrm{mmol}$ scale using 3-chlorobenzaldehyde (5.96 g, $42.4 \mathrm{mmol})$ and benzylamine $(4.32 \mathrm{~g}, 40.3 \mathrm{mmol})$. The product was purified by vacuum distillation $\left(143^{\circ} \mathrm{C}, 0.05 \mathrm{~mm} \mathrm{Hg}\right)$ to provide $2 \mathbf{2 l}(7.02 \mathrm{~g}, 30.6 \mathrm{mmol}, 76 \%)$ as a colorless liquid. IR (thin film, $\left.\mathrm{cm}^{-1}\right) 3063$ (s), 3028 (s), 2872 (s), 1645 (s), 1570 (s), 1452 (s), 1431 (s), 1373 (s), 1281 (m), 1215 (s), 1074 (s), 1028 (s); ${ }^{1} \mathrm{H}$ NMR (500 MHz, $\mathrm{CDCl}_{3}$ ) $\delta 8.34$ $(1 \mathrm{H}, \mathrm{d}, J=1.5 \mathrm{~Hz}, \mathrm{CH}=\mathrm{N}), 7.83-7.81(1 \mathrm{H}, \mathrm{m}, \operatorname{ArH}), 7.64-7.61(1 \mathrm{H}, \mathrm{m}, \mathrm{ArH}), 7.41-7.33(6 \mathrm{H}, \mathrm{m}, \mathrm{ArH})$, 7.30-7.28 (1H, m, ArH), $4.83\left(2 \mathrm{H}, \mathrm{s}, \mathrm{CH}_{2}-\mathrm{N}\right) ;{ }^{13} \mathrm{C}$ NMR $\left(101 \mathrm{MHz}, \mathrm{CDCl}_{3}\right) \delta 160.4,139.0,138.0,134.8$, 130.7, 129.9, 128.6, 128.1, 127.9, 127.2, 126.7, 65.0; LRMS (CI) m/z: $229.7(100 \%)[\mathrm{M}+\mathrm{H}]^{+}$.

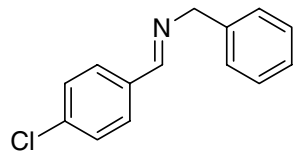

2m: Reaction was performed on a $41 \mathrm{mmol}$ scale using 4-chlorobenzaldehyde (5.90 $\mathrm{g}, 42.0 \mathrm{mmol})$ and benzylamine $(4.44 \mathrm{~g}, 41.1 \mathrm{mmol})$. The product was purified by vacuum distillation $\left(142^{\circ} \mathrm{C}, 0.05 \mathrm{~mm} \mathrm{Hg}\right)$ to provide $2 \mathrm{~m}(7.24 \mathrm{~g}, 31.5 \mathrm{mmol}, 77 \%)$ as a colorless liquid which slowly solidified to a white crystalline solid. $\mathrm{mp}=31$ $33^{\circ} \mathrm{C}$; IR (thin film, $\mathrm{cm}^{-1}$ ) 3028 (m), 2875 (m), 1643 (s), 1595 (s), 1489 (s), 1452 (s), 1375 (s), 1295 (s), 1087 (s), $1014(\mathrm{~s}), 860(\mathrm{~m}) ;{ }^{1} \mathrm{H}$ NMR $\left(500 \mathrm{MHz}, \mathrm{CDCl}_{3}\right) \delta 8.36(1 \mathrm{H}, \mathrm{s}, \mathrm{CH}=\mathrm{N}), 7.72(2 \mathrm{H}, \mathrm{d}, J=8.3 \mathrm{~Hz}$, $\mathrm{ArH}), 7.41-7.32$ (5H, m, ArH), 7.29-7.25 (2H, m, ArH), $4.82\left(2 \mathrm{H}, \mathrm{s}, \mathrm{CH}_{2}-\mathrm{N}\right) ;{ }^{13} \mathrm{C} \mathrm{NMR}\left(101 \mathrm{MHz}, \mathrm{CDCl}_{3}\right)$ $\delta 160.5,139.1,136.7,134.7,129.5,128.9,128.6,128.0,127.1,65.0 ;$ HRMS (ES) $\mathrm{m} / \mathrm{z}$ for $\mathrm{C}_{14} \mathrm{H}_{13} \mathrm{ClN}$ $[\mathrm{M}+\mathrm{H}]^{+}$calcd. 230.0736, found 230.0726 .

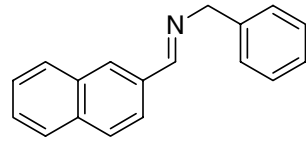

2n: Reaction was performed on a $40 \mathrm{mmol}$ scale using 2-naphthaldehyde $(6.25 \mathrm{~g}$, $40.0 \mathrm{mmol})$ and benzylamine $(4.32 \mathrm{~g}, 40.3 \mathrm{mmol})$. The product was purified by recrtystallization from hexanes to provide $2 \mathbf{n}(7.31 \mathrm{~g}, 29.8 \mathrm{mmol}, 74 \%)$ as a white crystalline solid. $\mathrm{mp}=84-86^{\circ} \mathrm{C}$; IR (thin film, $\mathrm{cm}^{-1}$ ) $3057(\mathrm{~m}), 3026(\mathrm{~m}), 2866(\mathrm{~s})$, $1638(\mathrm{~s}), 1495(\mathrm{~m}), 1451(\mathrm{~m}), 1350(\mathrm{~m}), 1045(\mathrm{~m}), 955(\mathrm{~m}) ;{ }^{1} \mathrm{H}$ NMR $\left(500 \mathrm{MHz}, \mathrm{CDCl}_{3}\right) \delta 8.56(1 \mathrm{H}, \mathrm{s}$, $\mathrm{CH}=\mathrm{N})$, 8.10-8.04 (2H, m, ArH), 7.92-7.84 (3H, m, ArH), 7.56-7.50 (2H, m, ArH), 7.41-7.35 (4H, m, $\mathrm{ArH}), 7.30-7.26(1 \mathrm{H}, \mathrm{m}, \mathrm{ArH}), 4.90\left(2 \mathrm{H}, \mathrm{s}, \mathrm{CH}_{2}-\mathrm{N}\right) ;{ }^{13} \mathrm{C}$ NMR $\left(101 \mathrm{MHz}, \mathrm{CDCl}_{3}\right) \delta 162.1,139.4,134.8$, 133.9, 133.1, 130.2, 128.7, 128.6, 128.5, 128.1, 127.9, 127.2, 127.1, 126.5, 124.0, 65.2; LRMS (CI) m/z: $245.7(100 \%)[\mathrm{M}+\mathrm{H}]^{+}$.

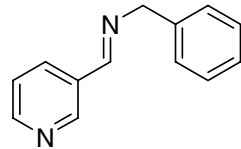

2o: Reaction was performed on a $41 \mathrm{mmol}$ scale using 3-pyridinecarboxaldehyde (4.56 $\mathrm{g}, 42.6 \mathrm{mmol})$ and benzylamine $(4.35 \mathrm{~g}, 40.6 \mathrm{mmol})$. The product was purified by vacuum distillation $\left(140^{\circ} \mathrm{C}, 0.05 \mathrm{~mm} \mathrm{Hg}\right)$ to provide $2 \mathrm{o}(4.61 \mathrm{~g}, 23.5 \mathrm{mmol}, 58 \%)$ as a colorless liquid. IR (thin film, $\left.\mathrm{cm}^{-1}\right) 3029(\mathrm{~m}), 2843(\mathrm{~m}), 1643(\mathrm{~s}), 1589(\mathrm{~m}), 1572(\mathrm{~m})$, $1452(\mathrm{~m}), 1420$ (s), $1376(\mathrm{~m}), 1325(\mathrm{~m}), 1187(\mathrm{~m}), 1027(\mathrm{~s}) ;{ }^{1} \mathrm{H}$ NMR $\left(500 \mathrm{MHz}, \mathrm{CDCl}_{3}\right) \delta 8.90(1 \mathrm{H}, \mathrm{d}, J=$ $1.5 \mathrm{~Hz}, \operatorname{ArH}), 8.66(1 \mathrm{H}, \mathrm{dd}, J=4.9,1.5 \mathrm{~Hz}, \mathrm{ArH}), 8.43(1 \mathrm{H}, \mathrm{s}, \mathrm{CH}=\mathrm{N}), 8.19-8.15(1 \mathrm{H}, \mathrm{m}, \operatorname{ArH}), 7.39-7.33$ $(5 \mathrm{H}, \mathrm{m}, \mathrm{ArH}), 7.30-7.26(1 \mathrm{H}, \mathrm{m}, \mathrm{ArH}), 4.86\left(2 \mathrm{H}, \mathrm{s}, \mathrm{CH}_{2}-\mathrm{N}\right) ;{ }^{13} \mathrm{C}$ NMR $\left(101 \mathrm{MHz}, \mathrm{CDCl}_{3}\right) \delta 158.5,151.1$, 149.9, 138.5, 134.1, 131.2, 128.1, 127.6, 126.7, 123.2, 64.7; HRMS (ES) $\mathrm{m} / \mathrm{z}$ for $\mathrm{C}_{13} \mathrm{H}_{13} \mathrm{~N}_{2}[\mathrm{M}+\mathrm{H}]^{+}$calcd. 197.1078, found 197.1078.

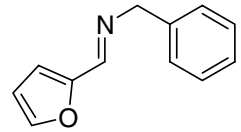

2p: Reaction was performed on a $41 \mathrm{mmol}$ scale using 2-furaldehyde ( $4.18 \mathrm{~g}, 43.5$ $\mathrm{mmol}$ ) and benzylamine $(4.41 \mathrm{~g}, 41.2 \mathrm{mmol})$. The product was purified by vacuum distillation $\left(110^{\circ} \mathrm{C}, 0.05 \mathrm{~mm} \mathrm{Hg}\right)$ to provide $2 \mathrm{p}(5.74 \mathrm{~g}, 31.0 \mathrm{mmol}, 75 \%)$ as a yellow liquid. IR (thin film, $\mathrm{cm}^{-1}$ ) 3094 (m), 2996 (m), 2836 (m), 1643 (s), 1580 (m), 1483 (s), 1452 (s), 1360 (s), 1273 (s), 1153 (s), 1017 (s); ${ }^{1} \mathrm{H}$ NMR (500 MHz, CDCl $)_{3} \delta 8.17$ (1H, s, CH=N), 7.53$7.51(1 \mathrm{H}, \mathrm{m}, \mathrm{ArH}), 7.36-7.31(4 \mathrm{H}, \mathrm{m}, \operatorname{ArH}), 7.29-7.25(1 \mathrm{H}, \mathrm{m}, \operatorname{ArH}), 6.78(1 \mathrm{H}, \mathrm{d}, J=3.4 \mathrm{~Hz}, \mathrm{ArH}), 6.48$ $(1 \mathrm{H}, \mathrm{dd}, J=3.4,1.5 \mathrm{~Hz}, \mathrm{ArH}), 4.78\left(2 \mathrm{H}, \mathrm{d}, J=1.0 \mathrm{~Hz}, \mathrm{CH}_{2}-\mathrm{N}\right) ;{ }^{13} \mathrm{C} \mathrm{NMR}\left(101 \mathrm{MHz}, \mathrm{CDCl}_{3}\right) \delta 151.4$, 150.2, 144.6, 138.7, 128.4, 128.1, 127.0, 114.0, 111.5, 65.0; LRMS (CI) m/z: $185.7(100 \%)[\mathrm{M}+\mathrm{H}]^{+}$. 


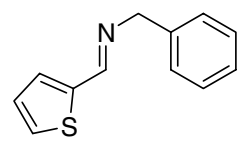

2q: Reaction was performed on a $41 \mathrm{mmol}$ scale using 2-thiophenecarboxaldehyde (4.80 $\mathrm{g}, 42.8 \mathrm{mmol})$ and benzylamine $(4.37 \mathrm{~g}, 40.8 \mathrm{mmol})$. The product was purified by vacuum distillation $\left(129^{\circ} \mathrm{C}, 0.05 \mathrm{~mm} \mathrm{Hg}\right)$ to provide $2 \mathrm{q}(5.30 \mathrm{~g}, 26.3 \mathrm{mmol}, 65 \%)$ as a yellow liquid which slowly solidifies to a yellow solid. $\mathrm{mp}=28-31^{\circ} \mathrm{C}$; IR (thin film, $\mathrm{cm}$ $\left.{ }^{1}\right) 3027(\mathrm{~m}), 2869(\mathrm{~m}), 1632(\mathrm{~s}), 1496(\mathrm{~m}), 1452(\mathrm{~m}), 1431(\mathrm{~s}), 1346(\mathrm{~m}), 1218(\mathrm{~m}), 1045(\mathrm{~m}), 859(\mathrm{~m}) ;{ }^{1} \mathrm{H}$ NMR (500 MHz, CDCl $)_{3} \delta 8.46(1 \mathrm{H}, \mathrm{s}, \mathrm{CH}=\mathrm{N}), 7.42-7.40(1 \mathrm{H}, \mathrm{m}, \mathrm{ArH}), 7.37-7.30(5 \mathrm{H}, \mathrm{m}, \mathrm{ArH}), 7.29-$ $7.25(1 \mathrm{H}, \mathrm{m}, \mathrm{ArH}), 7.08(1 \mathrm{H}, \mathrm{dd}, J=4.9,3.9 \mathrm{~Hz}, \mathrm{ArH}), 4.80\left(2 \mathrm{H}, \mathrm{s}, \mathrm{CH}_{2}-\mathrm{N}\right) ;{ }^{13} \mathrm{C} \mathrm{NMR}\left(101 \mathrm{MHz}, \mathrm{CDCl}_{3}\right)$ $\delta$ 155.1, 142.4, 139.0, 130.6, 129.0, 128.4, 128.0, 127.3, 127.0, 64.4; HRMS (ES) $\mathrm{m} / \mathrm{z}$ for $\mathrm{C}_{12} \mathrm{H}_{12} \mathrm{NS}$ $[\mathrm{M}+\mathrm{H}]^{+}$calcd. 202.0690, found 202.0681 .

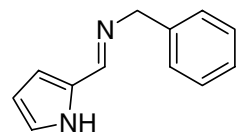

2r: Reaction was performed on a $40 \mathrm{mmol}$ scale using pyrrole-2-carboxaldehyde ( $3.80 \mathrm{~g}$, $40.0 \mathrm{mmol})$ and benzylamine $(4.32 \mathrm{~g}, 40.3 \mathrm{mmol})$. The product was purified by recrystallization from hexanes to provide $2 \mathbf{r}(6.12 \mathrm{~g}, 33.2 \mathrm{mmol}, 83 \%)$ as white needles. IR (thin film, $\mathrm{cm}^{-1}$ ) 3208 (s), 3167 (s), 3088 (m), 2830 (m), 1634 (s), 1422 (s), 1132 (m), $1028(\mathrm{~s}) ;{ }^{1} \mathrm{H}$ NMR $\left(500 \mathrm{MHz}, \mathrm{CDCl}_{3}\right) \delta 9.33(1 \mathrm{H}, \mathrm{brs}, \mathrm{NH}), 8.18(1 \mathrm{H}, \mathrm{s}, \mathrm{CH}=\mathrm{N}), 7.36-7.24(5 \mathrm{H}, \mathrm{m}, \mathrm{ArH})$, $6.84(1 \mathrm{H}, \mathrm{s}, \operatorname{ArH}), 6.52(1 \mathrm{H}, \mathrm{dd}, J=3.4,1.5 \mathrm{~Hz}, \operatorname{ArH}), 6.24(1 \mathrm{H}, \mathrm{dd}, J=3.4,2.9 \mathrm{~Hz}, \operatorname{ArH}), 4.72(2 \mathrm{H}, \mathrm{s}$, $\left.\mathrm{CH}_{2}-\mathrm{N}\right) ;{ }^{13} \mathrm{C}$ NMR $\left(101 \mathrm{MHz}, \mathrm{CDCl}_{3}\right) \delta 153.4,139.6,130.1,128.6,128.0,127.1,122.5,115.1,109.6,64.3$; LRMS (CI) m/z: $184.7(100 \%)[\mathrm{M}+\mathrm{H}]^{+}$.

\section{Phosphite Preparation. ${ }^{4}$}

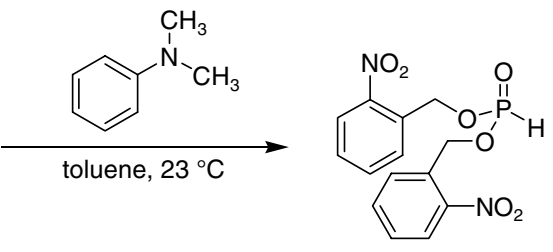

$3 h$<smiles>O=[N+]([O-])c1ccccc1COCc1ccccc1[N+](=O)[O-]</smiles>

3h: A flame-dried 500-mL 3-neck round bottomed flask equipped with a glass stopper, gas inlet adapter, rubber septum, and magnetic stir bar was charged with toluene (120 $\mathrm{mL}$ ). The glass stopper was replaced with an oven-dried $50 \mathrm{~mL}$ pressure-equalizing addition funnel equipped with a rubber septum. The addition funnel was charged with 2nitrobenzyl alcohol $(6.126 \mathrm{~g}, 40 \mathrm{mmol})$, toluene $(40 \mathrm{~mL})$, and $N, N$-dimethylaniline $(4.85$ g, $40 \mathrm{mmol})$. Phosphorus trichloride $(2.75 \mathrm{~g}, 20 \mathrm{mmol})$ was added to the $500 \mathrm{~mL}$ rbf. The mixture in the addition funnel was heated via a heat gun until homogeneous. This solution was added to the pot in small portions over $30 \mathrm{~min}$, re-heating as necessary. After $1 \mathrm{~h}, 2$-nitrobenzyl alcohol (3.06 g, $20 \mathrm{mmol})$ in warm toluene $(40 \mathrm{~mL})$ was added to the reaction over $15 \mathrm{~min}$ via the addition funnel. The reaction vessel was wrapped with aluminum foil to exclude light. After $12 \mathrm{~h}$, the reaction mixture was washed with deionized water $(3 \times 50 \mathrm{~mL})$, an aqueous solution of ammonia $(5 \mathrm{M}, 2$ X $50 \mathrm{~mL})$, deionized water $(2 \mathrm{X} 50 \mathrm{~mL})$, and brine $(50 \mathrm{~mL})$. The organic solution was dried over anhydrous sodium sulfate, filtered, and concentrated in vacuo. The resulting orange oil was dissolved in toluene $(80 \mathrm{~mL})$ and hexane $(30 \mathrm{~mL})$ was added to this solution slowly and with efficient mixing. The recrystallization was wrapped in aluminum foil to exclude light and allowed to stand at $23^{\circ} \mathrm{C}$ until the white solid product precipitated out of solution. It was then cooled to $4^{\circ} \mathrm{C}$ and kept at this temperature for $12 \mathrm{~h}$. The white solid was collected by vacuum filtration and was washed with hexanes $(120 \mathrm{~mL})$. This material was then dissolved in toluene $(100 \mathrm{~mL})$ by gently heating (temperature should not exceed $\left.50^{\circ} \mathrm{C}\right)$ until homogeneous. Hexane $(35 \mathrm{~mL})$ was added slowly and with efficient mixing to this warm solution and the recrystallization was carried out as before. The white solid was collected by vacuum filtration and washed with 40:60 hexanes:toluene $(120 \mathrm{~mL})$ followed by a hexanes wash $(20 \mathrm{~mL})$. Residual solvent was removed by high vacuum to provide $3 \mathbf{h}$ ( $3.67 \mathrm{~g}, 10.4 \mathrm{mmol}$, $52 \%$ ) as a white powder. $\mathrm{mp}=88-92^{\circ} \mathrm{C}$; IR (thin film, $\left.\mathrm{cm}^{-1}\right) 1613(\mathrm{w}), 1526(\mathrm{~s}), 1451(\mathrm{w}), 1344(\mathrm{~s}), 1256$ (s), $1092(\mathrm{~m}), 1053(\mathrm{~m}), 1042(\mathrm{~m}), 995(\mathrm{~m}) ;{ }^{1} \mathrm{H}$ NMR $\left(500 \mathrm{MHz}, \mathrm{CDCl}_{3}\right) \delta 8.16(2 \mathrm{H}, \mathrm{dd}, J=8.3,1.0 \mathrm{~Hz}$, $\operatorname{ArH}), 7.77(2 \mathrm{H}, \mathrm{d}, J=7.8 \mathrm{~Hz}, \operatorname{ArH}), 7.71(2 \mathrm{H}, \mathrm{dt}, J=1.0,7.8 \mathrm{~Hz}, \mathrm{ArH}), 7.56-7.51(2 \mathrm{H}, \mathrm{m}, \mathrm{ArH}), 7.17$ $(1 \mathrm{H}, \mathrm{d}, J=716 \mathrm{~Hz}, \mathrm{PH}), 5.62\left(2 \mathrm{H}, \mathrm{dd}, J=14.6,8.8 \mathrm{~Hz}, \mathrm{OCH}_{2}\right), 5.55\left(2 \mathrm{H}, \mathrm{dd}, J=14.6,8.3 \mathrm{~Hz}, \mathrm{OCH}_{2}\right) ;{ }^{13} \mathrm{C}$ NMR (101 MHz, $\left.\mathrm{CDCl}_{3}\right) \delta 146.9(\mathrm{~s}), 134.4(\mathrm{~s}), 132.1$ (d, $\left.J=7.3 \mathrm{~Hz}\right), 129.3(\mathrm{~s}), 128.8(\mathrm{~s}), 125.3(\mathrm{~s}), 64.3$ 
(d, $J=4.1 \mathrm{~Hz}) ;{ }^{31} \mathrm{P}$ NMR $\left(162 \mathrm{MHz}, \mathrm{CDCl}_{3}\right.$, proton-coupled) $\delta 8.72$ (ddd, $J=716,8.4,8.4 \mathrm{~Hz}$ ); HRMS (ES) $\mathrm{m} / \mathrm{z}$ for $\mathrm{C}_{14} \mathrm{H}_{14} \mathrm{~N}_{2} \mathrm{O}_{7} \mathrm{P}[\mathrm{M}+\mathrm{H}]^{+}$calcd. 353.0538, found 353.0551.

\section{Enantioselective Hydrophosphonylation.}
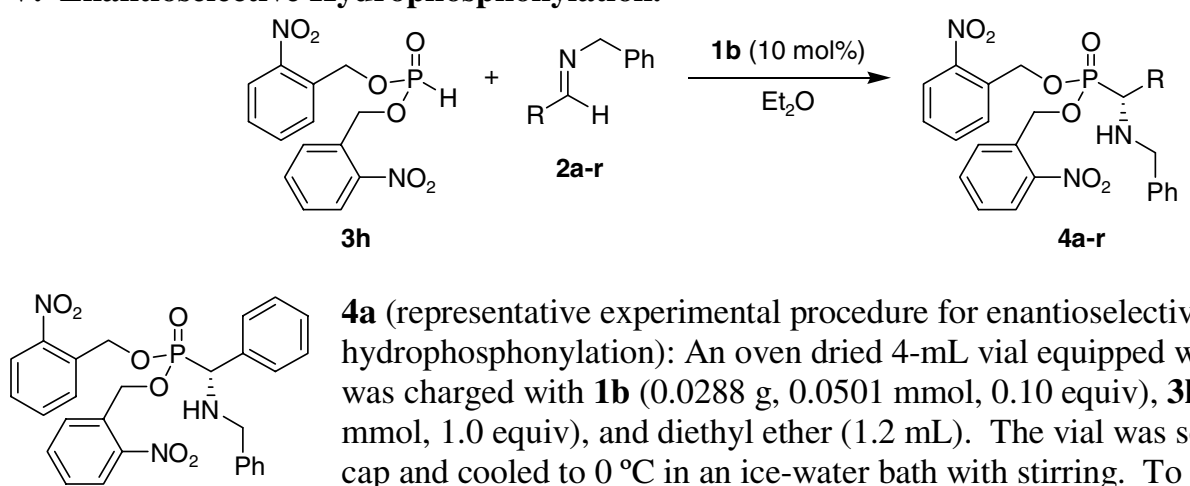

4a (representative experimental procedure for enantioselective hydrophosphonylation): An oven dried 4-mL vial equipped with a magnetic stir bar was charged with $\mathbf{1 b}(0.0288 \mathrm{~g}, 0.0501 \mathrm{mmol}, 0.10$ equiv), $\mathbf{3 h}(0.177 \mathrm{~g}, 0.501$ mmol, 1.0 equiv), and diethyl ether $(1.2 \mathrm{~mL})$. The vial was sealed with a plastic cap and cooled to $0^{\circ} \mathrm{C}$ in an ice-water bath with stirring. To this suspension was added $2 \mathbf{a}\left(0.0979 \mathrm{~g}, 0.501 \mathrm{mmol}, 1.0\right.$ equiv). The heterogeneous reaction was warmed to $4{ }^{\circ} \mathrm{C}$ (note: $3 \mathbf{h}$ is only sparingly soluble in the reaction mixture). After $72 \mathrm{~h}$, an aqueous solution of hydrochloric acid (1N, $0.5 \mathrm{~mL})$ and tetrahydrofuran $(0.5 \mathrm{~mL})$ were added, and this mixture was stirred at $4^{\circ} \mathrm{C}$ for $1 \mathrm{~h}$. The reaction was then partitioned between an aqueous solution of hydrochloric acid $(1 \mathrm{~N})$ and dichloromethane $(1: 1,40$ $\mathrm{mL}$ ). The layers were mixed well and separated. The aqueous layer was extracted with dichloromethane (5 $\mathrm{mL}$ ). The combined organic solution was washed with a saturated aqueous sodium carbonate solution (20 $\mathrm{mL})$. The sodium carbonate solution was back-extracted with dichloromethane $(2 \mathrm{X} 5 \mathrm{~mL})$, and the combined organic extracts were dried over sodium sulfate, filtered, and concentrated in vacuo. The resulting residue was purified via flash chromatography on silica gel (60:40 hexanes:ethyl acetate) to provide the product $4 \mathbf{a}$ as a very pale yellow solid $(0.239 \mathrm{~g}, 0.436 \mathrm{mmol}, 87 \%)$ in $98 \%$ ee. Chiral HPLC (Pirkle L-Leucine, 95:05 hexane:IPA, $1.0 \mathrm{~mL} / \mathrm{min}, \lambda=208 \mathrm{~nm}$ ) $t_{\mathrm{R}}$ (major) $=87.9 \mathrm{~min}, t_{\mathrm{R}}($ minor $)=100.1$ $\min ;[\alpha]^{25}=+24.4^{\circ}\left(\mathrm{c}=1.2, \mathrm{CHCl}_{3}\right) ; \mathrm{mp}=78-80^{\circ} \mathrm{C}$; IR (thin film, $\left.\mathrm{cm}^{-1}\right) 3304(\mathrm{~m}), 1526(\mathrm{~s}), 1343(\mathrm{~s})$, 1248 (s), 1055 (s), 1024 (s), 999 (s); ${ }^{1} \mathrm{H}-\mathrm{NMR}\left(500 \mathrm{MHz}, \mathrm{CDCl}_{3}\right) \delta 8.13(1 \mathrm{H}, \mathrm{dd}, J=8.3,1.5 \mathrm{~Hz}, \mathrm{ArH})$, $8.04(1 \mathrm{H}, \mathrm{dd}, J=8.1,1.2 \mathrm{~Hz}, \operatorname{ArH}), 7.71-7.62(2 \mathrm{H}, \mathrm{m}, \operatorname{ArH}), 7.51-7.34(8 \mathrm{H}, \mathrm{m}, \operatorname{ArH}), 7.30-7.21(6 \mathrm{H}, \mathrm{m}$, $\operatorname{ArH}), 5.63\left(1 \mathrm{H}, \mathrm{dd}, J=15,7.8 \mathrm{~Hz}, \mathrm{O}-\mathrm{CH}_{2}\right), 5.55\left(1 \mathrm{H}, \mathrm{dd}, J=15,6.8 \mathrm{~Hz}, \mathrm{O}-\mathbf{C H}_{2}\right), 5.34(1 \mathrm{H}, \mathrm{dd}, J=15$, $\left.7.3 \mathrm{~Hz}, \mathrm{O}-\mathrm{CH}_{2}\right), 5.22\left(1 \mathrm{H}, \mathrm{dd}, J=15,8.3 \mathrm{~Hz}, \mathrm{O}-\mathrm{CH}_{2}\right), 4.21(1 \mathrm{H}, \mathrm{d}, J=21 \mathrm{~Hz}, \mathrm{P}-\mathrm{CH}), 3.85(1 \mathrm{H}, \mathrm{d}, J=13$ $\left.\mathrm{Hz}, \mathrm{N}-\mathrm{CH}_{2}\right), 3.57\left(1 \mathrm{H}, \mathrm{d}, J=13 \mathrm{~Hz}, \mathrm{~N}-\mathrm{CH}_{2}\right), 2.41(1 \mathrm{H}, \mathrm{s}, \mathrm{N}-\mathrm{H}) ;{ }^{13} \mathrm{C}-\mathrm{NMR}\left(101 \mathrm{MHz}, \mathrm{CDCl}_{3}\right) \delta 146.8(\mathrm{~s})$, 146.6 (s), 139.0 (s), 135.2 (d, $J=2.3 \mathrm{~Hz}), 134.1(\mathrm{~s}), 134.0(\mathrm{~s}), 133.2$ (d, $J=6.9 \mathrm{~Hz}), 132.9$ (d, $J=6.9 \mathrm{~Hz})$, $129.0(\mathrm{~d}, J=2.3 \mathrm{~Hz}), 128.8(\mathrm{~s}), 128.8(\mathrm{~s}), 128.7(\mathrm{~s}), 128.7(\mathrm{~s}), 128.6(\mathrm{~s}), 128.6(\mathrm{~s}), 128.5(\mathrm{~s}), 128.3(\mathrm{~s})$, $127.4(\mathrm{~s}), 125.0(\mathrm{~s}), 124.9(\mathrm{~s}), 65.7(\mathrm{~d}, J=6.1 \mathrm{~Hz}), 64.9(\mathrm{~d}, J=6.1 \mathrm{~Hz}), 59.7(\mathrm{~d}, J=156 \mathrm{~Hz}), 51.2(\mathrm{~d}, J=$ $18.3 \mathrm{~Hz}$ ); ${ }^{31} \mathrm{P}-\mathrm{NMR}\left(162 \mathrm{MHz}, \mathrm{CDCl}_{3}\right) \delta 25.1$; HRMS (ES) $\mathrm{m} / \mathrm{z}$ for $\mathrm{C}_{28} \mathrm{H}_{27} \mathrm{~N}_{3} \mathrm{O}_{7} \mathrm{P}[\mathrm{M}+\mathrm{H}]^{+}$cacld. 548.1586, found 548.1596 .

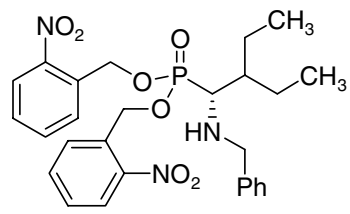

4b: Reaction was performed on a $0.48 \mathrm{mmol}$ scale at $4{ }^{\circ} \mathrm{C}$ for $24 \mathrm{~h}$ using 10 mol\% $1 \mathbf{b}(0.0273 \mathrm{~g}, 0.0475 \mathrm{mmol}), 3 \mathbf{h}(0.167 \mathrm{~g}, 0.475 \mathrm{mmol}), \mathbf{2 b}(0.0899 \mathrm{~g}$, $0.475 \mathrm{mmol})$, and diethyl ether $(1.2 \mathrm{~mL})$. The product was purified via flash chromatography on silica gel (70:30 to 60:40 hexanes:ethyl acetate gradient) to provide $\mathbf{4 b}$ as a very viscous pale yellow oil $(0.231 \mathrm{~g}, 0.426 \mathrm{mmol}, 90 \%)$ in $96 \%$ $e e$. Chiral HPLC (Chiralcel OD, 97:03 hexane:EtOH, $1.0 \mathrm{~mL} / \mathrm{min}, \lambda=208 \mathrm{~nm}$ ) $t_{\mathrm{R}}($ minor $)=28.6 \mathrm{~min}, t_{\mathrm{R}}($ major $)=34.8 \mathrm{~min} ;[\alpha]^{25}{ }_{\mathrm{D}}=-19.7^{\circ}\left(\mathrm{c}=1.1, \mathrm{CHCl}_{3}\right) ; \mathrm{IR}\left(\right.$ thin film, $\left.\mathrm{cm}^{-1}\right) 3351(\mathrm{~m})$, 3086 (m), 2963 (s), 2874 (s), 1530 (s), 1454 (m), 1344 (s), 1242 (s), 1042 (s), 1018 (s); ${ }^{1}$ H-NMR (500 $\left.\mathrm{MHz}, \mathrm{CDCl}_{3}\right) \delta 8.08(2 \mathrm{H}, \mathrm{dd}, J=8.3,1.0 \mathrm{~Hz}, \mathrm{ArH}), 8.10-8.07(2 \mathrm{H}, \mathrm{m}, \mathrm{ArH}), 7.65-7.61(2 \mathrm{H}, \mathrm{m}, \operatorname{ArH})$, 7.50-7.46 (2H, m, ArH), 7.33-7.21 (5H, m, ArH), 5.58-5.47 (4H, m, O-CH $)_{2}, 4.00(1 \mathrm{H}, \mathrm{d}, J=13 \mathrm{~Hz}, \mathrm{~N}-$ $\left.\mathrm{CH}_{2}\right), 3.88\left(1 \mathrm{H}, \mathrm{dd}, J=13,1.2 \mathrm{~Hz}, \mathrm{~N}-\mathrm{CH}_{2}\right), 3.24(1 \mathrm{H}, \mathrm{dd}, J=16,2.9 \mathrm{~Hz}, \mathrm{P}-\mathrm{CH}), 1.76-1.60(\mathrm{~m}, 2 \mathrm{H}$, $\left.\mathrm{CH}_{2} \mathrm{CH}_{3}\right), 1.57(1 \mathrm{H}, \mathrm{s}, \mathrm{N}-\mathbf{H}), 1.54-1.48\left(2 \mathrm{H}, \mathrm{m}, \mathrm{CH}_{2} \mathrm{CH}_{3}\right), 1.38-1.27\left(1 \mathrm{H}, \mathrm{m}, \mathrm{CH}\left(\mathrm{CH}_{2} \mathrm{CH}_{3}\right)_{2}\right), 0.92(3 \mathrm{H}, \mathrm{t}, J$ $\left.=7.6 \mathrm{~Hz}, \mathrm{CH}_{3}\right), 0.82\left(3 \mathrm{H}, \mathrm{t}, J=7.3 \mathrm{~Hz}, \mathrm{CH}_{3}\right) ;{ }^{13} \mathrm{C}-\mathrm{NMR}\left(101 \mathrm{MHz}, \mathrm{CDCl}_{3}\right) \delta 147.1(\mathrm{~s}), 147.1(\mathrm{~s}), 139.8$ (s), $134.1(\mathrm{~s}), 134.1$ (s), 133.0 (d, $J=6.0 \mathrm{~Hz}), 132.9$ (d, $J=6.4 \mathrm{~Hz}), 129.0$ (s), 129.0 (s), 129.0 (s), 128.9 $(\mathrm{s}), 128.5(\mathrm{~s}), 128.5(\mathrm{~s}), 127.3(\mathrm{~s}), 125.1(\mathrm{~s}), 125.1(\mathrm{~s}), 64.5(\mathrm{~d}, J=6.9 \mathrm{~Hz}), 64.4(\mathrm{~d}, J=6.4 \mathrm{~Hz}), 56.5(\mathrm{~d}, J=$ $143 \mathrm{~Hz}), 53.4(\mathrm{~d}, J=5.5 \mathrm{~Hz}), 43.2(\mathrm{~d}, J=5.5 \mathrm{~Hz}), 23.1(\mathrm{~d}, J=12.8 \mathrm{~Hz}), 22.8(\mathrm{~d}, J=2.3 \mathrm{~Hz}), 12.3(\mathrm{~s}), 12.3$ 
(s); ${ }^{31} \mathrm{P}-\mathrm{NMR}\left(162 \mathrm{MHz}, \mathrm{CDCl}_{3}\right) \delta 31.2$; HRMS (ES) $\mathrm{m} / \mathrm{z}$ for $\mathrm{C}_{27} \mathrm{H}_{33} \mathrm{~N}_{3} \mathrm{O}_{7} \mathrm{P}[\mathrm{M}+\mathrm{H}]^{+}$calcd. 542.2056, found 542.2062 .

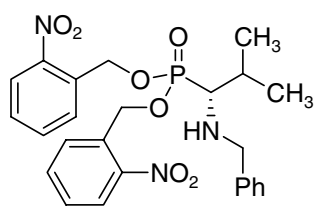

4c: Reaction was performed on a $0.50 \mathrm{mmol}$ scale at $4^{\circ} \mathrm{C}$ for $24 \mathrm{~h}$ using $10 \mathrm{~mol} \%$ 1b $(0.0289 \mathrm{~g}, 0.0503 \mathrm{mmol}), 3 \mathrm{~h}(0.177 \mathrm{~g}, 0.503 \mathrm{mmol}), 2 \mathbf{c}(0.0812 \mathrm{~g}, 0.503$ $\mathrm{mmol})$, and diethyl ether $(1.2 \mathrm{~mL})$. The product was purified via flash chromatography on silica gel (60:40 hexanes:ethyl acetate) to provide $4 \mathbf{c}$ as a very viscous pale yellow oil $(0.240 \mathrm{~g}, 0.467 \mathrm{mmol}, 93 \%)$ in $90 \%$ ee. Chiral HPLC (Chiralcel OD, 97:03 hexane:EtOH, $1.0 \mathrm{~mL} / \mathrm{min}, \lambda=208 \mathrm{~nm}$ ) $t_{\mathrm{R}}($ minor $)=41.7 \mathrm{~min}, t_{\mathrm{R}}($ major $)=49.5 \mathrm{~min}$; $[\alpha]^{25}=-15.1^{\circ}\left(\mathrm{c}=1.1, \mathrm{CHCl}_{3}\right)$; IR (thin film, $\left.\mathrm{cm}^{-1}\right) 3351(\mathrm{~m}), 3065(\mathrm{w}), 2965(\mathrm{~s}), 2874(\mathrm{~m}), 1530(\mathrm{~s})$, 1344 (s), 1246 (s), 1042 (s), 1020 (s); ${ }^{1} \mathrm{H}-\mathrm{NMR}\left(500 \mathrm{MHz}, \mathrm{CDCl}_{3}\right) \delta 8.08(2 \mathrm{H}, \mathrm{d}, J=8.3 \mathrm{~Hz}, \mathrm{ArH}), 7.73-$ 7.70 (2H, m, ArH), 7.64-7.61 (2H, m, ArH), 7.49-7.45 (2H, m, ArH), 7.33-7.22 (5H, m, ArH), 5.58-5.47 $\left(4 \mathrm{H}, \mathrm{m}, \mathrm{O}-\mathrm{CH}_{2}\right), 4.00\left(1 \mathrm{H}, \mathrm{d}, J=13 \mathrm{~Hz}, \mathrm{~N}-\mathrm{CH}_{2}\right), 3.90\left(1 \mathrm{H}, \mathrm{d}, J=13, \mathrm{~N}-\mathrm{CH}_{2}\right), 3.01(1 \mathrm{H}, \mathrm{dd}, J=14,3.9$ $\mathrm{Hz}, \mathrm{P}-\mathrm{CH}), 2.33-2.21\left(\mathrm{~m}, 1 \mathrm{H}, \mathrm{CH}\left(\mathrm{CH}_{3}\right)_{2}\right), 1.56(1 \mathrm{H}, \mathrm{s}, \mathrm{N}-\mathbf{H}), 1.10\left(3 \mathrm{H}, \mathrm{d}, J=6.8 \mathrm{~Hz}, \mathrm{CH}_{3}\right), 1.08(3 \mathrm{H}, \mathrm{d}, J$ $\left.=7.3 \mathrm{~Hz}, \mathrm{CH}_{3}\right) ;{ }^{13} \mathrm{C}-\mathrm{NMR}\left(101 \mathrm{MHz}, \mathrm{CDCl}_{3}\right) \delta 147.1(\mathrm{~s}), 147.1(\mathrm{~s}), 139.8(\mathrm{~s}), 134.1(\mathrm{~s}), 134.1(\mathrm{~s}), 133.0$ $(\mathrm{d}, J=6.1 \mathrm{~Hz}), 132.9(\mathrm{~d}, J=6.9 \mathrm{~Hz}), 129.0(\mathrm{~s}), 129.0(\mathrm{~s}), 128.9(\mathrm{~s}), 128.9(\mathrm{~s}), 128.5(\mathrm{~s}), 128.5(\mathrm{~s}), 127.3$ $(\mathrm{s}), 125.1(\mathrm{~s}), 125.1(\mathrm{~s}), 64.6(\mathrm{~d}, J=6.9 \mathrm{~Hz}), 64.4(\mathrm{~d}, J=6.1 \mathrm{~Hz}), 60.5(\mathrm{~d}, J=143 \mathrm{~Hz}), 53.6(\mathrm{~d}, J=5.3 \mathrm{~Hz})$, $29.3(\mathrm{~d}, J=5.3 \mathrm{~Hz}), 20.8(\mathrm{~d}, J=13 \mathrm{~Hz}), 18.2(\mathrm{~d}, J=3.8 \mathrm{~Hz}) ;{ }^{31} \mathrm{P}-\mathrm{NMR}\left(162 \mathrm{MHz}, \mathrm{CDCl}_{3}\right) \delta 30.1 ; \mathrm{HRMS}$ (ES) $\mathrm{m} / \mathrm{z}$ for $\mathrm{C}_{25} \mathrm{H}_{29} \mathrm{~N}_{3} \mathrm{O}_{7} \mathrm{P}[\mathrm{M}+\mathrm{H}]^{+}$calcd. 514.1743, found 514.1741.

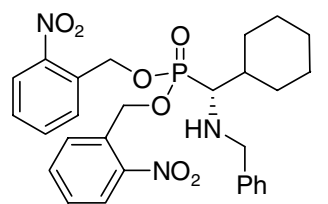

4d: Reaction was performed on a $0.48 \mathrm{mmol}$ scale at $4{ }^{\circ} \mathrm{C}$ at $24 \mathrm{~h}$ using $10 \mathrm{~mol} \%$ 1b $(0.0273 \mathrm{~g}, 0.0475 \mathrm{mmol}), 3 \mathbf{h}(0.167 \mathrm{~g}, 0.475 \mathrm{mmol}), \mathbf{2 d}(0.0957 \mathrm{~g}, 0.475$ $\mathrm{mmol})$, and diethyl ether $(1.2 \mathrm{~mL})$. The product was purified via flash chromatography on silica gel (60:40 hexanes:ethyl acetate) to provide $\mathbf{4 d}$ as a very viscous pale yellow oil $(0.238 \mathrm{~g}, 0.430 \mathrm{mmol}, 91 \%)$ in $90 \%$ ee. Chiral HPLC

(Chiralcel OD, 95:05 hexane:EtOH, $1.0 \mathrm{~mL} / \mathrm{min}, \lambda=208 \mathrm{~nm}) t_{\mathrm{R}}($ minor $)=20.8 \mathrm{~min}, t_{\mathrm{R}}($ major $)=26.4 \mathrm{~min}$; $[\alpha]^{25}{ }_{\mathrm{D}}=-11.0^{\circ}\left(\mathrm{c}=1.2, \mathrm{CHCl}_{3}\right)$; IR (thin film, $\left.\mathrm{cm}^{-1}\right) 3345(\mathrm{w}), 3086(\mathrm{w}), 2929(\mathrm{~s}), 2854(\mathrm{~s}), 1530(\mathrm{~s}), 1450$ (s), 1343 (s), 1242 (s), 1042 (s), 1019 (s); ${ }^{1} \mathrm{H}-\mathrm{NMR}\left(500 \mathrm{MHz}, \mathrm{CDCl}_{3}\right)$ \% 8.09-8.07 (2H, m, ArH), 7.737.69 (2H, m, ArH), 7.64-7.60 (2H, m, ArH), 7.49-7.45 (2H, m, ArH), 7.32-7.21 (5H, m, ArH), 5.58-5.46 $\left(4 \mathrm{H}, \mathrm{m}, \mathrm{O}-\mathrm{CH}_{2}\right), 4.00\left(1 \mathrm{H}, \mathrm{d}, J=13 \mathrm{~Hz}, \mathrm{~N}-\mathrm{CH}_{2}\right), 3.90\left(1 \mathrm{H}, \mathrm{dd}, J=13,1.2 \mathrm{~Hz}, \mathrm{~N}-\mathrm{CH}_{2}\right), 3.00(1 \mathrm{H}, \mathrm{dd}, J=$ $15,3.9 \mathrm{~Hz}, \mathrm{P}-\mathrm{CH}), 1.93-1.84\left(2 \mathrm{H}, \mathrm{m}, c-\mathrm{C}_{6} \mathbf{H}_{11}\right), 1.79-1.74\left(2 \mathrm{H}, \mathrm{m}, c-\mathrm{C}_{6} \mathbf{H}_{11}\right), 1.71-1.64\left(2 \mathrm{H}, \mathrm{m}, c-\mathrm{C}_{6} \mathbf{H}_{11}\right)$, $1.58(1 \mathrm{H}, \mathrm{s}, \mathrm{N}-\mathbf{H}), 1.50-1.41\left(1 \mathrm{H}, \mathrm{m}, c-\mathrm{C}_{6} \mathbf{H}_{11}\right), 1.31-1.10\left(4 \mathrm{H}, \mathrm{m}, c-\mathrm{C}_{6} \mathbf{H}_{11}\right) ;{ }^{13} \mathrm{C}-\mathrm{NMR}\left(101 \mathrm{MHz}, \mathrm{CDCl}_{3}\right) \delta$ 147.0 (s), 147.0 (s), 139.7 (s), 134.0 (s), 134.0 (s), 133.0 (d, $J=6.0 \mathrm{~Hz}), 132.9$ (d, $J=6.4 \mathrm{~Hz}), 128.9$ (s), $128.9(\mathrm{~s}), 128.9$ (s), $128.8(\mathrm{~s}), 128.4(\mathrm{~s}), 128.4(\mathrm{~s}), 127.3(\mathrm{~s}), 125.0(\mathrm{~s}), 125.1(\mathrm{~s}), 64.7$ (d, $J=6.4 \mathrm{~Hz}), 64.4$ $(\mathrm{d}, J=6.9 \mathrm{~Hz}), 60.5(\mathrm{~d}, J=144 \mathrm{~Hz}), 53.7(\mathrm{~d}, J=6.0 \mathrm{~Hz}), 39.5(\mathrm{~d}, J=5.0 \mathrm{~Hz}), 31.0(\mathrm{~d}, J=11.5 \mathrm{~Hz}), 28.5$ $(\mathrm{d}, J=4.1 \mathrm{~Hz}), 26.6(\mathrm{~s}), 26.4(\mathrm{~s}), 26.1(\mathrm{~s}) ;{ }^{31} \mathrm{P}-\mathrm{NMR}\left(162 \mathrm{MHz}, \mathrm{CDCl}_{3}\right) \delta 30.0 ; \mathrm{HRMS}(\mathrm{ES}) \mathrm{m} / \mathrm{z}$ for $\mathrm{C}_{28} \mathrm{H}_{33} \mathrm{~N}_{3} \mathrm{O}_{7} \mathrm{P}[\mathrm{M}+\mathrm{H}]^{+}$calcd. 554.2056, found 554.2062.

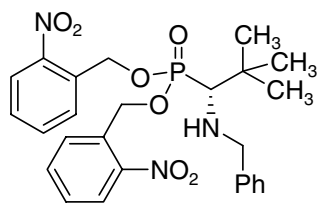

4e: Reaction was performed on a $0.50 \mathrm{mmol}$ scale at $4^{\circ} \mathrm{C}$ for $72 \mathrm{~h}$ using $10 \mathrm{~mol} \%$ 1b $(0.0287 \mathrm{~g}, 0.0500 \mathrm{mmol}), 3 \mathrm{~h}(0.176 \mathrm{~g}, 0.500 \mathrm{mmol}), \mathbf{2 e}(0.0876 \mathrm{~g}, 0.500$ $\mathrm{mmol})$, and diethyl ether $(1.2 \mathrm{~mL})$. The product was purified via flash chromatography on silica gel (60:40 hexanes:ethyl acetate) to provide $4 \mathbf{e}$ as a very viscous pale yellow oil $(0.219 \mathrm{~g}, 0.415 \mathrm{mmol}, 83 \%)$ in $93 \%$ ee. Chiral HPLC (Chiralcel OD, 95:05 hexane:EtOH, $1.0 \mathrm{~mL} / \mathrm{min}, \lambda=208 \mathrm{~nm}$ ) $t_{\mathrm{R}}($ minor $)=19.6 \mathrm{~min}, t_{\mathrm{R}}($ major $)=26.0 \mathrm{~min}$; $[\alpha]^{25}=-8.3^{\circ}\left(\mathrm{c}=1.2, \mathrm{CHCl}_{3}\right)$; IR (thin film, $\left.\mathrm{cm}^{-1}\right) 3362(\mathrm{w}), 3086(\mathrm{w}), 2957(\mathrm{~s}), 2870(\mathrm{~s}), 1530(\mathrm{~s}), 1342$ (s), 1242 (s), 1042 (s), 1015 (s); ${ }^{1} \mathrm{H}-\mathrm{NMR}\left(500 \mathrm{MHz}, \mathrm{CDCl}_{3}\right) \delta$ 8.09-8.04 (2H, m, ArH), 7.73-7.68 (2H, m, $\operatorname{ArH}), 7.64-7.57(2 \mathrm{H}, \mathrm{m}, \operatorname{ArH}), 7.49-7.43(2 \mathrm{H}, \mathrm{m}, \operatorname{ArH}), 7.33-7.22(5 \mathrm{H}, \mathrm{m}, \operatorname{ArH}), 5.53(2 \mathrm{H}, \mathrm{d}, J=7.8 \mathrm{~Hz}$, $\left.\mathrm{O}-\mathrm{CH}_{2}\right), 5.47\left(2 \mathrm{H}, \mathrm{d}, J=8.3 \mathrm{~Hz}, \mathrm{O}-\mathrm{CH}_{2}\right), 4.04\left(1 \mathrm{H}, \mathrm{dd}, J=13,1.5 \mathrm{~Hz}, \mathrm{~N}-\mathrm{CH}_{2}\right), 3.84(1 \mathrm{H}, \mathrm{d}, J=13 \mathrm{~Hz}, \mathrm{~N}-$ $\left.\mathrm{CH}_{2}\right), 2.83(1 \mathrm{H}, \mathrm{d}, J=15 \mathrm{~Hz}, \mathrm{P}-\mathrm{CH}), 1.49(1 \mathrm{H}, \mathrm{s}, \mathrm{N}-\mathrm{H}), 1.14\left(9 \mathrm{H}, \mathrm{s},\left(\mathrm{CH}_{3}\right)_{3}\right),{ }^{13} \mathrm{C}-\mathrm{NMR}(101 \mathrm{MHz}$, $\left.\mathrm{CDCl}_{3}\right) \delta 146.9(\mathrm{~s}), 146.8(\mathrm{~s}), 139.7(\mathrm{~s}), 133.9(\mathrm{~s}), 133.9(\mathrm{~s}), 132.8(\mathrm{~d}, J=6.1 \mathrm{~Hz}), 132.6(\mathrm{~d}, J=6.1 \mathrm{~Hz})$, $128.8(\mathrm{~s}), 128.8(\mathrm{~s}), 128.8(\mathrm{~s}), 128.8(\mathrm{~s}), 128.3(\mathrm{~s}), 128.2(\mathrm{~s}), 127.1(\mathrm{~s}), 124.9(\mathrm{~s}), 124.8(\mathrm{~s}), 65.4(\mathrm{~d}, J=132$ $\mathrm{Hz}), 64.7(\mathrm{~d}, J=3.1 \mathrm{~Hz}), 64.0(\mathrm{~d}, J=6.9 \mathrm{~Hz}), 55.3(\mathrm{~d}, J=3.8 \mathrm{~Hz}), 35.5(\mathrm{~d}, J=6.9 \mathrm{~Hz}), 27.6(\mathrm{~d}, J=6.9$ $\mathrm{Hz}) ;{ }^{31} \mathrm{P}-\mathrm{NMR}\left(162 \mathrm{MHz}, \mathrm{CDCl}_{3}\right) \delta 29.8$; HRMS (CI) m/z for $\mathrm{C}_{26} \mathrm{H}_{31} \mathrm{~N}_{3} \mathrm{O}_{7} \mathrm{P}[\mathrm{M}+\mathrm{H}]^{+}$calcd. 528.1899, found 528.1895 . 


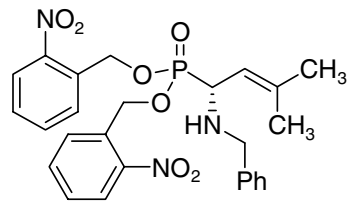

4f: Reaction was performed on a $2.19 \mathrm{mmol}$ scale at $4{ }^{\circ} \mathrm{C}$ using $10 \mathrm{~mol} \% \mathbf{1 b}$ (0.126 g, $0.219 \mathrm{mmol}), 3 \mathbf{3 h}(0.770 \mathrm{~g}, 2.19 \mathrm{mmol}), \mathbf{2 f}(0.379 \mathrm{~g}, 2.19 \mathrm{mmol})$, and diethyl ether $(5.3 \mathrm{~mL})$. After $72 \mathrm{~h}$, an aqueous solution of hydrochloric acid (1N, $2.2 \mathrm{~mL})$ and tetrahydrofuran $(2.2 \mathrm{~mL})$ were added, and this mixture was stirred at $4^{\circ} \mathrm{C}$ for $1 \mathrm{~h}$. The reaction was then partitioned between an aqueous solution of hydrochloric acid $(1 \mathrm{~N})$ and dichloromethane $(1: 1,160 \mathrm{~mL})$. The layers were mixed well and separated. The aqueous layer was extracted with dichloromethane $(20 \mathrm{~mL})$. The combined organic solution was washed with a saturated aqueous sodium carbonate solution $(80 \mathrm{~mL})$. The sodium carbonate solution was back-extracted with dichloromethane $(2 \times 20 \mathrm{~mL})$, and the combined organic extracts were dried over sodium sulfate, filtered, and concentrated in vacuo. The resulting residue was purified via flash chromatography on silica gel (60:40 to 50:50 hexanes:ethyl acetate gradient) to provide $\mathbf{4 f}$ as a very pale yellow crystalline solid (1.04 g, $1.98 \mathrm{mmol}, 91 \%$ ) in 82\% ee. Chiral HPLC (Chiralcel OD, 97:03 hexane:EtOH, $1.0 \mathrm{~mL} / \mathrm{min}, \lambda=208 \mathrm{~nm}) t_{\mathrm{R}}($ minor $)=48.4 \mathrm{~min}, t_{\mathrm{R}}($ major $)=55.1 \mathrm{~min} ;[\alpha]^{25}=+30.7^{\circ}(\mathrm{c}=$ $\left.1.0, \mathrm{CHCl}_{3}\right) ; \mathrm{mp}=98-101^{\circ} \mathrm{C}$. The product $(1.00 \mathrm{~g}, 1.90 \mathrm{mmol})$ was dissolved in hot ethyl acetate $(15 \mathrm{~mL})$ and hexane $(23 \mathrm{~mL})$ was added. The resulting solution was heated to reflux and then allowed to slowly cool to $\mathrm{rt}$ and then to $4^{\circ} \mathrm{C}$. The fine white needles were collected by vacuum filtration and washed with 60:40 hexanes:ethyl acetate $(40 \mathrm{~mL})$. The recrystallization was repeated to provide $\mathbf{4 f}(0.701 \mathrm{~g}, 1.33 \mathrm{mmol}$, 70\% recovery, 64\% overall from 2f) in 99\% ee. Chiral HPLC (Chiralcel OD, 97:03 hexane:EtOH, 1.0 $\mathrm{mL} / \mathrm{min}, \lambda=208 \mathrm{~nm}) t_{\mathrm{R}}($ minor $)=48.5 \mathrm{~min}, t_{\mathrm{R}}($ major $)=54.8 \mathrm{~min} ;[\alpha]_{\mathrm{D}}^{24}=+38.0^{\circ}\left(\mathrm{c}=1.1, \mathrm{CHCl}_{3}\right) ; \mathrm{mp}=$ 107-110 ${ }^{\circ} \mathrm{C}$; IR (thin film, $\mathrm{cm}^{-1}$ ) $3314(\mathrm{~m}), 3084(\mathrm{w}), 3028(\mathrm{~m}), 2972(\mathrm{~m}), 2855(\mathrm{~m}), 1528(\mathrm{~s}), 1343$ (s), 1242 (s), 1024 (s), 1001 (s); ${ }^{1} \mathrm{H}-\mathrm{NMR}\left(500 \mathrm{MHz}, \mathrm{CDCl}_{3}\right) \delta$ 8.14-8.09 (2H, m, ArH), 7.80-7.71 (2H, m, $\operatorname{ArH}), 7.67-7.61(2 \mathrm{H}, \mathrm{m}, \operatorname{ArH}), 7.51-7.45(2 \mathrm{H}, \mathrm{m}, \operatorname{ArH}), 7.30-7.22(5 \mathrm{H}, \mathrm{m}, \operatorname{ArH}), 5.63(1 \mathrm{H}, \mathrm{dd}, J=15,7.8$ $\left.\mathrm{Hz}, \mathrm{O}-\mathrm{CH}_{2}\right), 5.56\left(1 \mathrm{H}, \mathrm{dd}, J=15,6.8 \mathrm{~Hz}, \mathrm{O}-\mathrm{CH}_{2}\right), 5.58-5.48\left(2 \mathrm{H}, \mathrm{m}, \mathrm{O}-\mathrm{CH}_{2}\right), 5.21-5.16(1 \mathrm{H}, \mathrm{m}$, $\left.\mathrm{CHC}\left(\mathrm{CH}_{3}\right)_{2}\right), 3.93\left(1 \mathrm{H}, \mathrm{d}, J=13 \mathrm{~Hz}, \mathrm{~N}-\mathrm{CH}_{2}\right), 3.89(1 \mathrm{H}, \mathrm{dd}, J=18,10 \mathrm{~Hz}, \mathrm{P}-\mathrm{CH}), 3.69(1 \mathrm{H}, \mathrm{d}, J=13 \mathrm{~Hz}$, $\left.\mathrm{N}-\mathrm{CH}_{2}\right), 1.82\left(3 \mathrm{H}, \mathrm{d}, J=3.9 \mathrm{~Hz}, \mathrm{CH}_{3}\right), 1.80(1 \mathrm{H}, \mathrm{s}, \mathrm{N}-\mathbf{H}), 1.58\left(3 \mathrm{H}, \mathrm{dd}, J=3.4,1.0 \mathrm{~Hz}, \mathrm{CH}_{3}\right) ;{ }^{13} \mathrm{C}-\mathrm{NMR}$ $\left(101 \mathrm{MHz} \mathrm{CDCl}_{3}\right) \delta 146.7$ (s), 146.7 (s), 139.9 (d, $\left.J=15 \mathrm{~Hz}\right), 139.4(\mathrm{~s}), 134.0$ (s), $134.0(\mathrm{~s}), 133.2$ (d, $J=$ $6.4 \mathrm{~Hz}), 133.1$ (d, $J=6.4 \mathrm{~Hz}), 128.7$ (s), 128.7 (s), 128.5 (s), 128.5 (s), 128.4 (s), 128.4 (s), 127.2 (s), 125.0 (s), $125.0(\mathrm{~s}), 119.2(\mathrm{~d}, J=5.0 \mathrm{~Hz}), 65.2(\mathrm{~d}, J=6.0 \mathrm{~Hz}), 64.9(\mathrm{~d}, J=6.0 \mathrm{~Hz}), 53.5(\mathrm{~d}, J=160 \mathrm{~Hz}), 51.1(\mathrm{~d}$, $J=17.9 \mathrm{~Hz}$ ), $26.0(\mathrm{~d}, J=2.7 \mathrm{~Hz}), 18.7$ (d, $J=1.8 \mathrm{~Hz}) ;{ }^{31} \mathrm{P}-\mathrm{NMR}\left(162 \mathrm{MHz}, \mathrm{CDCl}_{3}\right) \delta 26.8$; HRMS (ES) $\mathrm{m} / \mathrm{z}$ for $\mathrm{C}_{26} \mathrm{H}_{29} \mathrm{~N}_{3} \mathrm{O}_{7} \mathrm{P}[\mathrm{M}+\mathrm{H}]^{+}$calcd. 526.1743, found 526.1725.

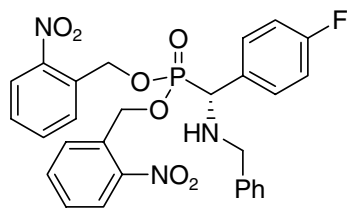

4g: Reaction was performed on a $0.51 \mathrm{mmol}$ scale at $4{ }^{\circ} \mathrm{C}$ for $72 \mathrm{~h}$ using 10 $\mathrm{mol} \% \mathbf{1 b}(0.0292 \mathrm{~g}, 0.0507 \mathrm{mmol}), 3 \mathrm{~h}(0.179 \mathrm{~g}, 0.507 \mathrm{mmol}), 2 \mathrm{~g}(0.108 \mathrm{~g}$, $0.507 \mathrm{mmol})$, and diethyl ether $(1.2 \mathrm{~mL})$. The product was purified via flash chromatography on silica gel (60:40 hexanes:ethyl acetate) to provide $\mathbf{4 g}$ as a very pale yellow crystalline solid $(0.254 \mathrm{~g}, 0.449 \mathrm{mmol}, 89 \%)$ in $98 \%$ ee. Chiral HPLC (Chiralpak AD, 70:30 hexane:IPA, $1.0 \mathrm{~mL} / \mathrm{min}, \lambda=208 \mathrm{~nm}) t_{\mathrm{R}}($ major $)=$ $35.2 \mathrm{~min}, t_{\mathrm{R}}$ (minor) $=55.2 \mathrm{~min} ;[\alpha]_{\mathrm{D}}^{25}=+19.9^{\circ}\left(\mathrm{c}=1.4, \mathrm{CHCl}_{3}\right) ; \mathrm{mp}=80-82^{\circ} \mathrm{C}$; IR (thin film, $\left.\mathrm{cm}^{-1}\right) 3314$ (w), 3067 (w), 2923 (w), 1527 (s), 1508 (s), 1449 (m), 1342 (s), 1246 (s), 1024 (s), 999 (s); ${ }^{1}$ H-NMR (500 $\left.\mathrm{MHz}_{\mathrm{CDCl}}\right) \delta 8.13(1 \mathrm{H}, \mathrm{dd}, J=8.3,1.0 \mathrm{~Hz}, \mathrm{ArH}), 8.05(1 \mathrm{H}, \mathrm{dd}, J=8.3,1.0 \mathrm{~Hz}, \operatorname{ArH}), 7.68-7.62(2 \mathrm{H}, \mathrm{m}$, ArH), 7.53-7.48 (2H, m, ArH), 7.45-7.39 (3H, m, ArH), 7.31-7.19 (6H, m, ArH), 7.09-7.05 (2H, m, ArH), $5.62\left(1 \mathrm{H}, \mathrm{dd}, J=15,7.8 \mathrm{~Hz}, \mathrm{O}-\mathrm{CH}_{2}\right), 5.54\left(1 \mathrm{H}, \mathrm{dd}, J=15,7.3 \mathrm{~Hz}, \mathrm{O}-\mathrm{CH}_{2}\right), 5.36(1 \mathrm{H}, \mathrm{dd}, J=15,7.3 \mathrm{~Hz}$, O-CH $\left.\mathbf{H}_{2}\right), 5.28\left(1 \mathrm{H}, \mathrm{dd}, J=15,8.3 \mathrm{~Hz}, \mathrm{O}-\mathrm{CH}_{2}\right), 4.18(1 \mathrm{H}, \mathrm{d}, J=21 \mathrm{~Hz}, \mathrm{P}-\mathrm{CH}), 3.83(1 \mathrm{H}, \mathrm{d}, J=13 \mathrm{~Hz}, \mathrm{~N}-$ $\left.\mathrm{CH}_{2}\right), 3.54\left(1 \mathrm{H}, \mathrm{d}, J=13 \mathrm{~Hz}, \mathrm{~N}-\mathrm{CH}_{2}\right), 2.38(1 \mathrm{H}, \mathrm{s}, \mathrm{N}-\mathbf{H}) ;{ }^{13} \mathrm{C}-\mathrm{NMR}\left(101 \mathrm{MHz}, \mathrm{CDCl}_{3}\right) \delta 162.8(\mathrm{dd}, J=$ 247, $3.7 \mathrm{~Hz}), 146.9$ (s), 146.8 (s), 138.8 (s), 134.1 (s), 134.0 (s), 133.0 (d, J = 6.9 Hz), 132.6 (d, $J=6.4$ $\mathrm{Hz}), 131.0$ (dd, $J=3.2,3.2 \mathrm{~Hz}), 130.4$ (dd, $J=8.0,6.6 \mathrm{~Hz}), 128.9$ (s), 128.9 (s), 128.7 (s), 128.6 (s), 128.5 $(\mathrm{s}), 128.5(\mathrm{~s}), 127.5(\mathrm{~s}), 125.1(\mathrm{~s}), 125.0(\mathrm{~s}), 115.9(\mathrm{dd}, J=22,1.8 \mathrm{~Hz}), 65.7$ (d, $J=6.0 \mathrm{~Hz}), 65.0(\mathrm{~d}, J=6.0$ $\mathrm{Hz}), 58.9(\mathrm{~d}, J=157 \mathrm{~Hz}), 51.1(\mathrm{~d}, J=18 \mathrm{~Hz}) ;{ }^{31} \mathrm{P}-\mathrm{NMR}\left(162 \mathrm{MHz}, \mathrm{CDCl}_{3}\right) \delta 24.9 ; \mathrm{HRMS}(\mathrm{ES}) \mathrm{m} / \mathrm{z}$ for $\mathrm{C}_{28} \mathrm{H}_{26} \mathrm{FN}_{3} \mathrm{O}_{7} \mathrm{P}[\mathrm{M}+\mathrm{H}]^{+}$cacld. 566.1492, found 566.1490.

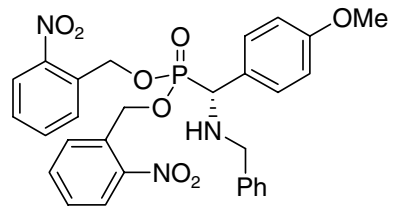

4h: Reaction was performed on a $0.50 \mathrm{mmol}$ scale at $4{ }^{\circ} \mathrm{C}$ for $48 \mathrm{~h}$ using 10 $\mathrm{mol} \% \mathbf{1 b}(0.0287 \mathrm{~g}, 0.0500 \mathrm{mmol}), 3 \mathrm{~h}(0.176 \mathrm{~g}, 0.500 \mathrm{mmol}), \mathbf{2 h}(0.113 \mathrm{~g}$, $0.500 \mathrm{mmol})$, and diethyl ether $(1.2 \mathrm{~mL})$. The product was purified via flash chromatography on silica gel (55:45 hexanes:ethyl acetate) to provide $\mathbf{4 h}$ as a 
very pale yellow crystalline solid $(0.261 \mathrm{~g}, 0.452 \mathrm{mmol}, 90 \%)$ in $96 \%$ ee. Chiral HPLC (Chiralpak AD, 70:30 hexane:IPA, $1.0 \mathrm{~mL} / \mathrm{min}, \lambda=208 \mathrm{~nm}) t_{\mathrm{R}}($ major $)=58.4 \mathrm{~min}, t_{\mathrm{R}}($ minor $)=66.3 \mathrm{~min} ;[\alpha]^{25}{ }_{\mathrm{D}}=+33.4^{\circ}(\mathrm{c}$ $=1.0, \mathrm{CHCl}_{3}$ ); $\mathrm{mp}=100-102^{\circ} \mathrm{C}$; IR (thin film, $\mathrm{cm}^{-1}$ ) $3320(\mathrm{w}), 3065(\mathrm{w}), 2937(\mathrm{w}), 1527(\mathrm{~s}), 1453(\mathrm{~m})$, 1341 (s), 1250 (s), $1086(\mathrm{~m}), 1025$ (s), 999 (s); ${ }^{1} \mathrm{H}-\mathrm{NMR}\left(500 \mathrm{MHz}, \mathrm{CDCl}_{3}\right) \delta 8.13(1 \mathrm{H}, \mathrm{dd}, J=8.1,1.2$ $\mathrm{Hz}, \operatorname{ArH}), 8.04(1 \mathrm{H}, \mathrm{dd}, J=8.1,1.2 \mathrm{~Hz}, \operatorname{ArH}), 7.73-7.71(1 \mathrm{H}, \mathrm{m}, \operatorname{ArH}), 7.62-7.66(1 \mathrm{H}, \mathrm{m}, \operatorname{ArH}), 7.52-7.47$ $(2 \mathrm{H}, \mathrm{m}, \mathrm{ArH}), 7.43-7.39(1 \mathrm{H}, \mathrm{m}, \mathrm{ArH}), 7.36-7.21(8 \mathrm{H}, \mathrm{m}, \operatorname{ArH}), 6.92-6.88(2 \mathrm{H}, \mathrm{m}, \mathrm{ArH}), 5.62(1 \mathrm{H}, \mathrm{dd}, J$ $\left.=15,7.8 \mathrm{~Hz}, \mathrm{O}-\mathrm{CH}_{2}\right), 5.54\left(1 \mathrm{H}, \mathrm{dd}, J=15,6.8 \mathrm{~Hz}, \mathrm{O}-\mathrm{CH}_{2}\right), 5.33\left(1 \mathrm{H}, \mathrm{dd}, J=15,6.8 \mathrm{~Hz}, \mathrm{O}-\mathrm{CH}_{2}\right), 5.23$ $\left(1 \mathrm{H}, \mathrm{dd}, J=15,7.8 \mathrm{~Hz}, \mathrm{O}-\mathrm{CH}_{2}\right), 4.14(1 \mathrm{H}, \mathrm{d}, J=21 \mathrm{~Hz}, \mathrm{P}-\mathrm{CH}), 3.84\left(1 \mathrm{H}, \mathrm{d}, J=13 \mathrm{~Hz}, \mathrm{~N}-\mathrm{CH}_{2}\right), 3.84(3 \mathrm{H}$, s, O-CH $\left.\mathbf{H}_{3}\right), 3.56\left(1 \mathrm{H}, \mathrm{d}, J=13 \mathrm{~Hz}, \mathrm{~N}-\mathrm{CH}_{2}\right), 2.34(1 \mathrm{H}, \mathrm{s}, \mathrm{N}-\mathbf{H}) ;{ }^{13} \mathrm{C}-\mathrm{NMR}\left(101 \mathrm{MHz}, \mathrm{CDCl}_{3}\right) \delta 159.7(\mathrm{~d}, J$ $=2.8 \mathrm{~Hz}), 146.6(\mathrm{~s}), 146.5(\mathrm{~s}), 139.0(\mathrm{~s}), 134.0(\mathrm{~s}), 133.9(\mathrm{~s}), 133.2(\mathrm{~d}, J=6.9 \mathrm{~Hz}), 132.8(\mathrm{~d}, J=6.4 \mathrm{~Hz})$, $129.8(\mathrm{~d}, J=6.4 \mathrm{~Hz}), 128.7(\mathrm{~s}), 128.5(\mathrm{~s}), 128.5(\mathrm{~s}), 128.4(\mathrm{~s}), 128.4(\mathrm{~s}), 128.3(\mathrm{~s}), 128.2(\mathrm{~s}), 126.8(\mathrm{~d}, J=$ $2.7 \mathrm{~Hz}), 124.9(\mathrm{~s}), 124.8(\mathrm{~s}), 114.2(\mathrm{~d}, J=2.3 \mathrm{~Hz}), 65.5(\mathrm{~d}, J=6.0 \mathrm{~Hz}), 64.8(\mathrm{~d}, J=6.0 \mathrm{~Hz}), 58.8(\mathrm{~d}, J=$ $157 \mathrm{~Hz}), 55.3(\mathrm{~s}), 50.9(\mathrm{~d}, J=18 \mathrm{~Hz}) ;{ }^{31} \mathrm{P}-\mathrm{NMR}\left(162 \mathrm{MHz}, \mathrm{CDCl}_{3}\right) \delta 25.5 ; \mathrm{HRMS}$ (ES) m/z for $\mathrm{C}_{29} \mathrm{H}_{29} \mathrm{~N}_{3} \mathrm{O}_{8} \mathrm{P}[\mathrm{M}+\mathrm{H}]^{+}$cacld. 578.1692, found 578.1687.

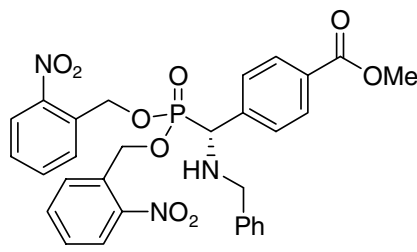

4i: Reaction was performed on a $0.50 \mathrm{mmol}$ scale at $23^{\circ} \mathrm{C}$ for $48 \mathrm{~h}$ using $10 \mathrm{~mol} \% \mathbf{1 b}(0.0287 \mathrm{~g}, 0.0500 \mathrm{mmol}), 3 \mathbf{h}(0.176 \mathrm{~g}, 0.500 \mathrm{mmol}), \mathbf{2 i}(0.127$ $\mathrm{g}, 0.500 \mathrm{mmol})$, and diethyl ether $(1.2 \mathrm{~mL})$. The product was purified via flash chromatography on silica gel (55:45 hexanes:ethyl acetate) to provide $4 \mathbf{i}$ as a very pale yellow crystalline solid $(0.237 \mathrm{~g}, 0.391 \mathrm{mmol}, 78 \%)$ in 96\% ee. Chiral HPLC (Chiralpak AD, 70:30 hexane:IPA, $1.0 \mathrm{~mL} / \mathrm{min}, \lambda=$ $208 \mathrm{~nm}) t_{\mathrm{R}}($ major $)=46.6 \mathrm{~min}, t_{\mathrm{R}}($ minor $)=54.2 \mathrm{~min} ;[\alpha]_{\mathrm{D}}^{25}=+33.4^{\circ}\left(\mathrm{c}=1.2, \mathrm{CHCl}_{3}\right) ; \mathrm{mp}=79-82^{\circ} \mathrm{C}$; IR (thin film, $\mathrm{cm}^{-1}$ ) $3318(\mathrm{~m}), 3063(\mathrm{~m}), 2951$ (m), 1715 (s), 1529 (s), $1497(\mathrm{~s}), 1343$ (s), 1248 (s), 1111 (s), $1018(\mathrm{~s}) ;{ }^{1} \mathrm{H}-\mathrm{NMR}\left(500 \mathrm{MHz}, \mathrm{CDCl}_{3}\right) \delta 8.13(1 \mathrm{H}, \mathrm{d}, J=8.8 \mathrm{~Hz}, \mathrm{ArH}), 8.05-8.03$ (3H, m, ArH), 7.66-7.61 $(2 \mathrm{H}, \mathrm{m}, \mathrm{ArH}), 7.53-7.40(5 \mathrm{H}, \mathrm{m}, \mathrm{ArH}), 7.31-7.18(6 \mathrm{H}, \mathrm{m}, \mathrm{ArH}), 5.62\left(1 \mathrm{H}, \mathrm{dd}, J=15,7.8 \mathrm{~Hz}, \mathrm{O}-\mathrm{CH}_{2}\right)$, $5.54\left(1 \mathrm{H}, \mathrm{dd}, J=15,7.3 \mathrm{~Hz}, \mathrm{O}-\mathrm{CH}_{2}\right), 5.37\left(1 \mathrm{H}, \mathrm{dd}, J=15,6.8 \mathrm{~Hz}, \mathrm{O}-\mathrm{CH}_{2}\right), 5.28(1 \mathrm{H}, \mathrm{dd}, J=15,8.3 \mathrm{~Hz}$, $\left.\mathrm{O}-\mathrm{CH}_{2}\right), 4.27(1 \mathrm{H}, \mathrm{d}, J=21 \mathrm{~Hz}, \mathrm{P}-\mathrm{CH}), 3.95\left(3 \mathrm{H}, \mathrm{s}, \mathrm{O}-\mathrm{CH}_{3}\right), 3.83\left(1 \mathrm{H}, \mathrm{d}, J=13 \mathrm{~Hz}, \mathrm{~N}-\mathrm{CH}_{2}\right), 3.54(1 \mathrm{H}, \mathrm{d}$, $\left.J=13 \mathrm{~Hz}, \mathrm{~N}-\mathrm{CH}_{2}\right), 2.45(1 \mathrm{H}, \mathrm{s}, \mathrm{N}-\mathrm{H}) ;{ }^{13} \mathrm{C}-\mathrm{NMR}\left(101 \mathrm{MHz}, \mathrm{CDCl}_{3}\right) \delta 166.7(\mathrm{~s}), 146.8(\mathrm{~s}), 146.7(\mathrm{~s}), 140.5$ $(\mathrm{d}, J=2.3 \mathrm{~Hz}), 138.6(\mathrm{~s}), 134.1(\mathrm{~s}), 134.0(\mathrm{~s}), 132.8(\mathrm{~d}, J=6.9 \mathrm{~Hz}), 132.4(\mathrm{~d}, J=6.1 \mathrm{~Hz}), 130.3(\mathrm{~d}, J=3.1$ $\mathrm{Hz}), 130.1(\mathrm{~s}), 130.1(\mathrm{~s}), 128.9(\mathrm{~s}), 128.9(\mathrm{~s}), 128.8(\mathrm{~s}), 128.7(\mathrm{~s}), 128.6(\mathrm{~s}), 128.4(\mathrm{~s}), 127.5(\mathrm{~s}), 125.0(\mathrm{~s})$, $125.0(\mathrm{~s}), 65.7(\mathrm{~d}, J=5.3 \mathrm{~Hz}), 65.0(\mathrm{~d}, J=6.1 \mathrm{~Hz}), 59.5(\mathrm{~d}, J=155 \mathrm{~Hz}), 52.3(\mathrm{~s}), 51.2(\mathrm{~d}, J=18 \mathrm{~Hz}) ;{ }^{31} \mathrm{P}-$ NMR (162 MHz, $\left.\mathrm{CDCl}_{3}\right) \delta 24.3$; HRMS (ES) $\mathrm{m} / \mathrm{z}$ for $\mathrm{C}_{30} \mathrm{H}_{29} \mathrm{~N}_{3} \mathrm{O}_{9} \mathrm{P}[\mathrm{M}+\mathrm{H}]^{+}$cacld. 606.1641, found 606.1633 .

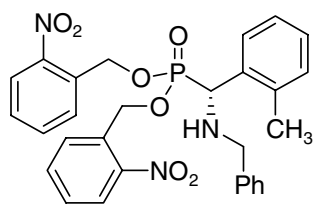

4j: Reaction was performed on a $0.49 \mathrm{mmol}$ scale at $4{ }^{\circ} \mathrm{C}$ for $72 \mathrm{~h}$ using $10 \mathrm{~mol} \%$ 1b (0.0282 g, $0.0491 \mathrm{mmol}), 3 \mathbf{h}(0.173 \mathrm{~g}, 0.491 \mathrm{mmol}), \mathbf{2 j}$ (0.103 g, $0.491 \mathrm{mmol})$, and diethyl ether $(1.2 \mathrm{~mL})$. The product was purified via flash chromatography on silica gel (65:35 hexanes:ethyl acetate) to provide $\mathbf{4} \mathbf{j}$ as a very viscous pale yellow oil $(0.224 \mathrm{~g}, 0.398 \mathrm{mmol}, 81 \%)$ in $92 \%$ ee. Chiral HPLC (Pirkle L-Leucine, 99:01 hexane:EtOH, $1.0 \mathrm{~mL} / \mathrm{min}, \lambda=208 \mathrm{~nm}) t_{\mathrm{R}}($ major $)=123.2 \mathrm{~min}, t_{\mathrm{R}}($ minor $)=136.5 \mathrm{~min} ;[\alpha]^{25}{ }_{\mathrm{D}}=+25.6^{\circ}(\mathrm{c}=$ 0.9, $\mathrm{CHCl}_{3}$ ); IR (thin film, $\mathrm{cm}^{-1}$ ) 3314 (m), 3065 (m), $2944(\mathrm{~m}), 1530$ (s), 1452 (s), 1344 (s), 1248 (s), 1198 (s), $1086(\mathrm{~m}), 1024(\mathrm{~s}) ;{ }^{1} \mathrm{H}-\mathrm{NMR}\left(500 \mathrm{MHz}, \mathrm{CDCl}_{3}\right) \delta 8.13(1 \mathrm{H}, \mathrm{dd}, J=8.3,1.0 \mathrm{~Hz}, \mathrm{ArH}), 8.03(1 \mathrm{H}, \mathrm{dd}, J$ $=8.3,1.0 \mathrm{~Hz}, \operatorname{ArH}), 7.72-7.62(3 \mathrm{H}, \mathrm{m}, \mathrm{ArH}), 7.51-7.38(3 \mathrm{H}, \mathrm{m}, \mathrm{ArH}), 7.32-7.14(9 \mathrm{H}, \mathrm{m}, \operatorname{ArH}), 5.63(1 \mathrm{H}$, dd, $\left.J=15,7.8 \mathrm{~Hz}, \mathrm{O}-\mathrm{CH}_{2}\right), 5.56\left(1 \mathrm{H}, \mathrm{dd}, J=15,7.3 \mathrm{~Hz}, \mathrm{O}-\mathrm{CH}_{2}\right), 5.31\left(1 \mathrm{H}, \mathrm{dd}, J=15,6.8 \mathrm{~Hz}, \mathrm{O}-\mathbf{C H}_{2}\right)$, $5.15\left(1 \mathrm{H}, \mathrm{dd}, J=15,8.3 \mathrm{~Hz}, \mathrm{O}-\mathrm{CH}_{2}\right), 4.49(1 \mathrm{H}, \mathrm{d}, J=21 \mathrm{~Hz}, \mathrm{P}-\mathrm{CH}), 3.85\left(1 \mathrm{H}, \mathrm{d}, J=13 \mathrm{~Hz}, \mathrm{~N}-\mathrm{CH}_{2}\right), 3.55$ $\left(1 \mathrm{H}, \mathrm{d}, J=13 \mathrm{~Hz}, \mathrm{~N}-\mathrm{CH}_{2}\right), 2.48(1 \mathrm{H}, \mathrm{s}, \mathrm{N}-\mathbf{H}), 2.18\left(3 \mathrm{H}, \mathrm{s}, \operatorname{ArCH} \mathbf{H}_{3}\right) ;{ }^{13} \mathrm{C}-\mathrm{NMR}\left(101 \mathrm{MHz}, \mathrm{CDCl}_{3}\right) \delta 146.8$ (s), 146.6 (s), 139.0 (s), 137.5 (d, $J=7.6 \mathrm{~Hz}), 134.1(\mathrm{~s}), 134.0(\mathrm{~s}), 133.4(\mathrm{~d}, J=1.5 \mathrm{~Hz}), 133.2$ (d, $J=6.9$ $\mathrm{Hz}), 132.8(\mathrm{~d}, J=6.1 \mathrm{~Hz}), 130.8(\mathrm{~d}, J=1.5 \mathrm{~Hz}), 128.8(\mathrm{~s}), 128.6(\mathrm{~s}), 128.6(\mathrm{~s}), 128.5(\mathrm{~s}), 128.5(\mathrm{~s}), 128.3$ (s), $128.1(\mathrm{~d}, J=3.8 \mathrm{~Hz}), 127.5(\mathrm{~d}, J=3.8 \mathrm{~Hz}), 127.4(\mathrm{~s}), 126.8(\mathrm{~d}, J=3.1 \mathrm{~Hz}), 125.0(\mathrm{~s}), 124.9(\mathrm{~s}), 65.6$ $(\mathrm{d}, J=5.3 \mathrm{~Hz}), 64.8(\mathrm{~d}, J=6.1 \mathrm{~Hz}), 54.6(\mathrm{~d}, J=157 \mathrm{~Hz}), 51.0(\mathrm{~d}, J=18 \mathrm{~Hz}), 19.7(\mathrm{~s}) ;{ }^{31} \mathrm{P}-\mathrm{NMR}(162$ $\mathrm{MHz}, \mathrm{CDCl}_{3}$ ) $\delta 26.1$; HRMS (ES) $\mathrm{m} / \mathrm{z}$ for $\mathrm{C}_{29} \mathrm{H}_{29} \mathrm{~N}_{3} \mathrm{O}_{7} \mathrm{P}[\mathrm{M}+\mathrm{H}]^{+}$cacld. 562.1743, found 562.1752.<smiles>O=[N+]([O-])c1ccccc1COP(=O)(O)C(NCc1ccccc1)c1ccccc1Cl</smiles>

4k: Reaction was performed on a $0.50 \mathrm{mmol}$ scale at $4{ }^{\circ} \mathrm{C}$ for $72 \mathrm{~h}$ using $20 \mathrm{~mol} \%$ 1b $(0.0575 \mathrm{~g}, 0.100 \mathrm{mmol}), 3 \mathrm{~h}(0.176 \mathrm{~g}, 0.500 \mathrm{mmol}), 2 \mathrm{k}(0.115 \mathrm{~g}, 0.500 \mathrm{mmol})$, 
and diethyl ether $(1.2 \mathrm{~mL})$. The product was purified via flash chromatography on silica gel (67:33 hexanes:ethyl acetate) to provide $4 \mathbf{k}$ as a very viscous pale yellow oil $(0.153 \mathrm{~g}, 0.263 \mathrm{mmol}, 52 \%)$ in $96 \%$ $e e$. Chiral HPLC (Chiralpak AD, 70:30 hexane:IPA, $1.0 \mathrm{~mL} / \mathrm{min}, \lambda=208 \mathrm{~nm}) t_{\mathrm{R}}($ major) $=35.5 \mathrm{~min}$, $t_{\mathrm{R}}($ minor $)=44.0 \mathrm{~min} ;[\alpha]_{\mathrm{D}}^{25}=+4.7^{\circ}\left(\mathrm{c}=1.1, \mathrm{CHCl}_{3}\right)$; IR (thin film, $\left.\mathrm{cm}^{-1}\right) 3306(\mathrm{~m}), 3065(\mathrm{~m}), 2942(\mathrm{~m})$, 1530 (s), 1447 (s), 1343 (s), 1248 (s), 1198 (m), 1086 (s), 1024 (s), 999 (s); ${ }^{1} \mathrm{H}-\mathrm{NMR}\left(500 \mathrm{MHz}, \mathrm{CDCl}_{3}\right) \delta$ $8.15(1 \mathrm{H}, \mathrm{dd}, J=8.3,1.0 \mathrm{~Hz}, \operatorname{ArH}), 8.06(1 \mathrm{H}, \mathrm{dd}, J=8.3,1.0 \mathrm{~Hz}, \operatorname{ArH}), 7.78-7.63(3 \mathrm{H}, \mathrm{m}, \operatorname{ArH}), 7.53-$ $7.47(2 \mathrm{H}, \mathrm{m}, \mathrm{ArH}), 7.44-7.34(4 \mathrm{H}, \mathrm{m}, \mathrm{ArH}), 7.31-7.21(6 \mathrm{H}, \mathrm{m}, \mathrm{ArH}), 5.72(1 \mathrm{H}, \mathrm{dd}, J=15,7.3 \mathrm{~Hz}, \mathrm{O}-$ $\left.\mathrm{CH}_{2}\right), 5.62\left(1 \mathrm{H}, \mathrm{dd}, J=15,6.8 \mathrm{~Hz}, \mathrm{O}-\mathrm{CH}_{2}\right), 5.37\left(1 \mathrm{H}, \mathrm{dd}, J=15,6.8 \mathrm{~Hz}, \mathrm{O}-\mathrm{CH}_{2}\right), 5.24(1 \mathrm{H}, \mathrm{dd}, J=15,7.8$ $\left.\mathrm{Hz}, \mathrm{O}-\mathrm{CH}_{2}\right), 4.93(1 \mathrm{H}, \mathrm{d}, J=22 \mathrm{~Hz}, \mathrm{P}-\mathrm{CH}), 3.79\left(1 \mathrm{H}, \mathrm{d}, J=13 \mathrm{~Hz}, \mathrm{~N}-\mathrm{CH}_{2}\right), 3.56(1 \mathrm{H}, \mathrm{d}, J=13 \mathrm{~Hz}, \mathrm{~N}-$ $\left.\mathrm{CH}_{2}\right), 2.45(1 \mathrm{H}, \mathrm{s}, \mathrm{N}-\mathbf{H}) ;{ }^{13} \mathrm{C}-\mathrm{NMR}\left(101 \mathrm{MHz}, \mathrm{CDCl}_{3}\right) \delta 146.7$ (s), $146.4(\mathrm{~s}), 138.8(\mathrm{~s}), 134.9(\mathrm{~d}, J=8.7$ $\mathrm{Hz}), 134.1$ (s), 134.0 (s), $134.0(\mathrm{~s}), 133.5(\mathrm{~s}), 133.1$ (d, $J=6.9 \mathrm{~Hz}), 132.8(\mathrm{~d}, J=6.8 \mathrm{~Hz}), 129.8$ (d, $J=2.3)$, $129.5(\mathrm{~d}, J=3.2 \mathrm{~Hz}), 129.3(\mathrm{~d}, J=4.1 \mathrm{~Hz}), 128.7(\mathrm{~s}), 128.6(\mathrm{~s}), 128.5(\mathrm{~s}), 128.5(\mathrm{~s}), 128.1(\mathrm{~s}), 127.7(\mathrm{~d}, J$ $=3.2 \mathrm{~Hz}), 127.4(\mathrm{~s}), 125.0(\mathrm{~s}), 124.9(\mathrm{~s}), 65.9(\mathrm{~d}, J=5.5 \mathrm{~Hz}), 64.8(\mathrm{~d}, J=6.0 \mathrm{~Hz}), 55.2(\mathrm{~d}, J=159 \mathrm{~Hz})$, $51.4\left(\mathrm{~d}, J=18 \mathrm{~Hz}\right.$ ); ${ }^{31} \mathrm{P}-\mathrm{NMR}\left(162 \mathrm{MHz}, \mathrm{CDCl}_{3}\right) \delta 24.6$; HRMS (ES) $\mathrm{m} / \mathrm{z}_{\text {for }} \mathrm{C}_{28} \mathrm{H}_{26} \mathrm{ClN}_{3} \mathrm{O}_{7} \mathrm{P}[\mathrm{M}+\mathrm{H}]^{+}$ cacld. 582.1197, found 582.1202.

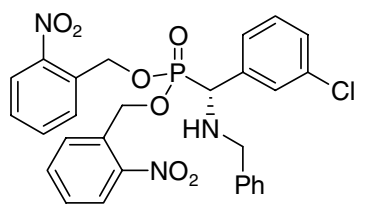

4I: Reaction was performed on a $0.50 \mathrm{mmol}$ scale at $23^{\circ} \mathrm{C}$ for $48 \mathrm{~h}$ using 10 $\mathrm{mol} \% \mathbf{1 b}(0.0287 \mathrm{~g}, 0.0500 \mathrm{mmol}), 3 \mathrm{~h}(0.176 \mathrm{~g}, 0.500 \mathrm{mmol}), 2 \mathrm{l}(0.115 \mathrm{~g}$, $0.500 \mathrm{mmol})$, and diethyl ether $(1.2 \mathrm{~mL})$. The product was purified via flash chromatography on silica gel (60:40 hexanes:ethyl acetate) to provide $\mathbf{4 I}$ as a very viscous pale yellow oil $(0.242 \mathrm{~g}, 0.415 \mathrm{mmol}, 83 \%)$ in $98 \%$ ee. Chiral HPLC (Chiralpak AD, 87:13 hexane:EtOH, $1.0 \mathrm{~mL} / \mathrm{min}, \lambda=208 \mathrm{~nm}) t_{\mathrm{R}}($ minor $)=71.6 \mathrm{~min}, t_{\mathrm{R}}($ major $)=$ $76.5 \mathrm{~min} ;[\alpha]^{25}{ }_{\mathrm{D}}=+22.7^{\circ}\left(\mathrm{c}=1.0, \mathrm{CHCl}_{3}\right)$; IR (thin film, $\left.\mathrm{cm}^{-1}\right) 3300(\mathrm{~m}), 3064(\mathrm{~m}), 2944(\mathrm{w}), 1531(\mathrm{~s})$, 1455 (s), 1344 (s), 1247 (s), 1197 (s), 1085 (s), 1022 (s), 998 (s); ${ }^{1} \mathrm{H}-\mathrm{NMR}\left(500 \mathrm{MHz}, \mathrm{CDCl}_{3}\right) \delta 8.13$ (1H, $\mathrm{d}, J=8.3 \mathrm{~Hz}, \operatorname{ArH}), 8.07(1 \mathrm{H}, \mathrm{dd}, J=8.3,1.0 \mathrm{~Hz}, \operatorname{ArH}), 7.68-7.63(2 \mathrm{H}, \mathrm{m}, \operatorname{ArH}), 7.56-7.41(4 \mathrm{H}, \mathrm{m}, \operatorname{ArH})$, 7.35-7.19 (9H, m, ArH), $5.62\left(1 \mathrm{H}, \mathrm{dd}, J=15,7.8 \mathrm{~Hz}, \mathrm{O}-\mathrm{CH}_{2}\right), 5.55\left(1 \mathrm{H}, \mathrm{dd}, J=15,6.8 \mathrm{~Hz}, \mathrm{O}-\mathrm{CH}_{2}\right), 5.38$ $\left(1 \mathrm{H}, \mathrm{dd}, J=15,6.8 \mathrm{~Hz}, \mathrm{O}-\mathrm{CH}_{2}\right), 5.31\left(1 \mathrm{H}, \mathrm{dd}, J=15,7.8 \mathrm{~Hz}, \mathrm{O}-\mathrm{CH}_{2}\right), 4.18(1 \mathrm{H}, \mathrm{d}, J=21 \mathrm{~Hz}, \mathrm{P}-\mathrm{CH}), 3.85$ $\left(1 \mathrm{H}, \mathrm{d}, J=13 \mathrm{~Hz}, \mathrm{~N}-\mathrm{CH}_{2}\right), 3.56\left(1 \mathrm{H}, \mathrm{d}, J=13 \mathrm{~Hz}, \mathrm{~N}-\mathrm{CH}_{2}\right), 2.41(1 \mathrm{H}, \mathrm{s}, \mathrm{N}-\mathrm{H}) ;{ }^{13} \mathrm{C}-\mathrm{NMR}(101 \mathrm{MHz}$, $\left.\mathrm{CDCl}_{3}\right) \delta 146.8(\mathrm{~s}), 146.6(\mathrm{~s}), 138.6(\mathrm{~s}), 137.5(\mathrm{~d}, J=2.8 \mathrm{~Hz}), 134.8(\mathrm{~d}, J=2.8 \mathrm{~Hz}), 134.2(\mathrm{~s}), 134.1(\mathrm{~s})$, $132.8(\mathrm{~d}, J=6.9 \mathrm{~Hz}), 132.5(\mathrm{~d}, J=6.9), 130.2(\mathrm{~d}, J=2.8), 128.9(\mathrm{~s}), 128.8(\mathrm{~s}), 128.8(\mathrm{~d}, J=6.9 \mathrm{~Hz}), 128.7$ (d, $J=3.2 \mathrm{~Hz}), 128.6(\mathrm{~s}), 128.6(\mathrm{~s}), 128.4(\mathrm{~s}), 128.4(\mathrm{~s}), 127.5(\mathrm{~s}), 126.8(\mathrm{~d}, J=6.0 \mathrm{~Hz}), 125.1(\mathrm{~s}), 125.0$ $(\mathrm{s}), 65.7(\mathrm{~d}, J=6.0 \mathrm{~Hz}), 65.0(\mathrm{~d}, J=5.5 \mathrm{~Hz}), 59.2(\mathrm{~d}, J=156 \mathrm{~Hz}), 51.2(\mathrm{~d}, J=18 \mathrm{~Hz}) ;{ }^{31} \mathrm{P}-\mathrm{NMR}(162$ $\left.\mathrm{MHz}, \mathrm{CDCl}_{3}\right) \delta$ 24.3; HRMS (ES) $\mathrm{m} / \mathrm{z}$ for $\mathrm{C}_{28} \mathrm{H}_{26} \mathrm{ClN}_{3} \mathrm{O}_{7} \mathrm{P}[\mathrm{M}+\mathrm{H}]^{+}$cacld. 582.1197, found 582.1201.

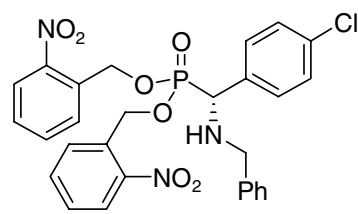

4m: Reaction was performed on a $0.50 \mathrm{mmol}$ scale at $4{ }^{\circ} \mathrm{C}$ for $72 \mathrm{~h}$ using 10 $\mathrm{mol} \% \mathbf{1 b}(0.0287 \mathrm{~g}, 0.0500 \mathrm{mmol}), 3 \mathbf{h}(0.176 \mathrm{~g}, 0.500 \mathrm{mmol}), \mathbf{2 m}(0.115 \mathrm{~g}$, $0.500 \mathrm{mmol})$, and diethyl ether $(1.2 \mathrm{~mL})$. The product was purified via flash chromatography on silica gel (60:40 hexanes:ethyl acetate) to provide $\mathbf{4 m}$ as a very pale yellow crystalline solid $(0.253 \mathrm{~g}, 0.434 \mathrm{mmol}, 87 \%)$ in $99 \%$ ee. Chiral HPLC (Chiralpak AD, 70:30 hexane:IPA, $1.0 \mathrm{~mL} / \mathrm{min}, \lambda=208 \mathrm{~nm}$ ) $t_{\mathrm{R}}($ major $)=38.6 \mathrm{~min}, t_{\mathrm{R}}($ minor $)=58.4 \mathrm{~min} ;[\alpha]^{25}{ }_{\mathrm{D}}=+29.7^{\circ}\left(\mathrm{c}=1.1, \mathrm{CHCl}_{3}\right) ; \mathrm{mp}=98-101^{\circ} \mathrm{C} ; \mathrm{IR}$ (thin film, $\left.\mathrm{cm}^{-1}\right) 3314(\mathrm{~m}), 3065(\mathrm{~m}), 2943(\mathrm{~m}), 1526(\mathrm{~s}), 1451(\mathrm{~m}), 1343(\mathrm{~s}), 1248(\mathrm{~s}), 1198(\mathrm{~m}), 1088(\mathrm{~m}), 1015(\mathrm{~s})$, 999 (s); ${ }^{1} \mathrm{H}-\mathrm{NMR}\left(500 \mathrm{MHz}, \mathrm{CDCl}_{3}\right) \delta 8.13(1 \mathrm{H}, \mathrm{d}, J=7.8 \mathrm{~Hz}, \mathrm{ArH}), 8.05(1 \mathrm{H}, \mathrm{dd}, J=8.3,1.5 \mathrm{~Hz}, \mathrm{ArH})$, 7.66-7.62 (2H, m, ArH), 7.53-7.42 (3H, m, ArH), 7.38-7.19 (10H, m, ArH), 5.62 (1H, dd, $J=15,7.8 \mathrm{~Hz}$, $\left.\mathrm{O}-\mathrm{CH}_{2}\right), 5.54\left(1 \mathrm{H}, \mathrm{dd}, J=15,6.8 \mathrm{~Hz}, \mathrm{O}-\mathrm{CH}_{2}\right), 5.37\left(1 \mathrm{H}, \mathrm{dd}, J=15,7.3 \mathrm{~Hz}, \mathrm{O}-\mathrm{CH}_{2}\right), 5.30(1 \mathrm{H}, \mathrm{dd}, J=15$, $\left.7.8 \mathrm{~Hz}, \mathrm{O}-\mathrm{CH}_{2}\right), 4.17(1 \mathrm{H}, \mathrm{d}, J=21 \mathrm{~Hz}, \mathrm{P}-\mathrm{CH}), 3.83\left(1 \mathrm{H}, \mathrm{d}, J=13 \mathrm{~Hz}, \mathrm{~N}-\mathbf{C H}_{2}\right), 3.53(1 \mathrm{H}, \mathrm{d}, J=13 \mathrm{~Hz}, \mathrm{~N}-$ $\left.\mathrm{CH}_{2}\right), 2.38(1 \mathrm{H}, \mathrm{s}, \mathrm{N}-\mathrm{H}) ;{ }^{13} \mathrm{C}-\mathrm{NMR}\left(101 \mathrm{MHz}, \mathrm{CDCl}_{3}\right) \delta 146.7$ (s), 146.6 (s), 138.6 (s), 134.2 (d, $J=3.7$ $\mathrm{Hz}), 134.0(\mathrm{~s}), 133.9(\mathrm{~s}), 133.8(\mathrm{~d}, J=2.7 \mathrm{~Hz}), 132.9(\mathrm{~d}, J=6.9 \mathrm{~Hz}), 132.4(\mathrm{~d}, J=6.4 \mathrm{~Hz}), 130.0(\mathrm{~d}, J=$ $6.4 \mathrm{~Hz}), 129.0(\mathrm{~s}), 129.0(\mathrm{~s}), 128.8(\mathrm{~s}), 128.8(\mathrm{~s}), 128.5(\mathrm{~s}), 128.4(\mathrm{~s}), 128.3(\mathrm{~s}), 127.4(\mathrm{~s}), 125.0(\mathrm{~s}), 124.9$ (s), $65.6(\mathrm{~d}, J=6.0 \mathrm{~Hz}), 64.9(\mathrm{~d}, J=6.0 \mathrm{~Hz}), 58.8(\mathrm{~d}, J=156 \mathrm{~Hz}), 51.0(\mathrm{~d}, J=18 \mathrm{~Hz}) ;{ }^{31} \mathrm{P}-\mathrm{NMR}(162$ $\left.\mathrm{MHz}, \mathrm{CDCl}_{3}\right) \delta$ 24.6; HRMS (ES) $\mathrm{m} / \mathrm{z}$ for $\mathrm{C}_{28} \mathrm{H}_{26} \mathrm{ClN}_{3} \mathrm{O}_{7} \mathrm{P}[\mathrm{M}+\mathrm{H}]^{+}$cacld. 582.1197, found 582.1194.

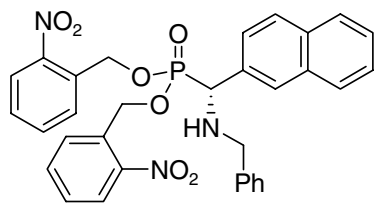

4n: Reaction was performed on a $0.50 \mathrm{mmol}$ scale at $4{ }^{\circ} \mathrm{C}$ for $72 \mathrm{~h}$ using 10 $\mathrm{mol} \% \mathbf{1 b}(0.0287 \mathrm{~g}, 0.0500 \mathrm{mmol}), 3 \mathbf{h}(0.176 \mathrm{~g}, 0.500 \mathrm{mmol}), 2 \mathrm{n}(0.123 \mathrm{~g}$, 
$0.500 \mathrm{mmol})$, and diethyl ether $(1.2 \mathrm{~mL})$. The product was purified via flash chromatography on silica gel (67:33 hexanes:ethyl acetate) to provide $\mathbf{4 n}$ as a very pale yellow crystalline solid $(0.257 \mathrm{~g}, 0.430 \mathrm{mmol}$, $86 \%$ ) in $98 \%$ ee. Chiral HPLC (Chiralpak AD, 70:30 hexane:IPA, $1.0 \mathrm{~mL} / \mathrm{min}, \lambda=208 \mathrm{~nm}) t_{\mathrm{R}}($ major $)=$ $57.7 \mathrm{~min}, t_{\mathrm{R}}($ minor $)=70.8 \mathrm{~min} ;[\alpha]^{25}{ }_{\mathrm{D}}=+41.7^{\circ}\left(\mathrm{c}=1.0, \mathrm{CHCl}_{3}\right) ; \mathrm{mp}=116-119^{\circ} \mathrm{C}$; IR (thin film, $\left.\mathrm{cm}^{-1}\right)$ 3306 (w), 3059 (w), 2942 (w), 1526 (s), 1451 (w), 1343 (s), 1246 (s), 1198 (w), 1086 (m), 1053 (s), 1022 (s); ${ }^{1} \mathrm{H}-\mathrm{NMR}\left(500 \mathrm{MHz}, \mathrm{CDCl}_{3}\right) \delta 8.12(1 \mathrm{H}, \mathrm{dd}, J=6.8,1.5 \mathrm{~Hz}, \mathrm{ArH}), 7.94(1 \mathrm{H}, \mathrm{dd}, J=8.3,1.5 \mathrm{~Hz}, \mathrm{ArH})$, 7.89-7.77 (4H, m, ArH), 7.68-7.65 (1H, m, ArH), 7.61-7.45 (5H, m, ArH), 7.31-7.21 (6H, m, ArH), 7.19$7.16(1 \mathrm{H}, \mathrm{m}, \operatorname{ArH}), 7.12-7.09(1 \mathrm{H}, \mathrm{m}, \operatorname{ArH}), 5.66\left(1 \mathrm{H}, \mathrm{dd}, J=15,7.8 \mathrm{~Hz}, \mathrm{O}-\mathrm{CH}_{2}\right), 5.57(1 \mathrm{H}, \mathrm{dd}, J=15$, $\left.7.3 \mathrm{~Hz}, \mathrm{O}-\mathrm{CH}_{2}\right), 5.34\left(1 \mathrm{H}, \mathrm{dd}, J=15,6.8 \mathrm{~Hz}, \mathrm{O}-\mathrm{CH}_{2}\right), 5.28\left(1 \mathrm{H}, \mathrm{dd}, J=15,7.8 \mathrm{~Hz}, \mathrm{O}-\mathrm{CH}_{2}\right), 4.37(1 \mathrm{H}, \mathrm{d}, J$ $=21 \mathrm{~Hz}, \mathrm{P}-\mathrm{CH}), 3.88\left(1 \mathrm{H}, \mathrm{d}, J=13 \mathrm{~Hz}, \mathrm{~N}-\mathrm{CH}_{2}\right), 3.60(1 \mathrm{H}, \mathrm{d}, J=13 \mathrm{~Hz}, \mathrm{~N}-\mathrm{CH} 2), 2.53(1 \mathrm{H}, \mathrm{s}, \mathrm{N}-\mathbf{H}) ;{ }^{13} \mathrm{C}-$ NMR (101 MHz, CDCl $\left.{ }_{3}\right) \delta 146.7$ (s), $146.4(\mathrm{~s}), 138.9(\mathrm{~s}), 134.0(\mathrm{~s}), 133.7$ (s), 133.3 (d, $\left.J=1.5 \mathrm{~Hz}\right), 133.2$ (d, $J=2.3), 133.0(\mathrm{~d}, J=6.1), 132.6(\mathrm{~d}, J=3.0 \mathrm{~Hz}), 132.5(\mathrm{~d}, J=6.1 \mathrm{~Hz}), 128.8(\mathrm{~s}), 128.7(\mathrm{~s}), 128.5(\mathrm{~s})$, $128.5(\mathrm{~s}), 128.4(\mathrm{~s}), 128.3(\mathrm{~s}), 128.2(\mathrm{~s}), 128.2(\mathrm{~s}), 127.9(\mathrm{~s}), 127.8(\mathrm{~s}), 127.3(\mathrm{~s}), 126.5(\mathrm{~s}), 126.4(\mathrm{~s}), 125.8$ $(\mathrm{d}, J=4.6), 124.9(\mathrm{~s}), 124.7(\mathrm{~s}), 65.6(\mathrm{~d}, J=6.1 \mathrm{~Hz}), 64.8(\mathrm{~d}, J=5.3 \mathrm{~Hz}), 59.7(\mathrm{~d}, J=156 \mathrm{~Hz}), 51.1(\mathrm{~d}, J=$ $18 \mathrm{~Hz}) ;{ }^{31} \mathrm{P}-\mathrm{NMR}\left(162 \mathrm{MHz}, \mathrm{CDCl}_{3}\right) \delta 25.1$; HRMS (ES) $\mathrm{m} / \mathrm{z}$ for $\mathrm{C}_{32} \mathrm{H}_{29} \mathrm{~N}_{3} \mathrm{O}_{7} \mathrm{P}[\mathrm{M}+\mathrm{H}]^{+}$cacld. 598.1743, found 598.1757 .

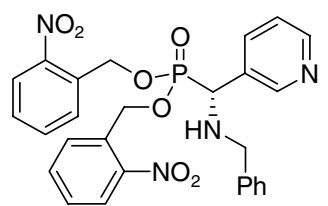

4o: Reaction was performed on a $0.50 \mathrm{mmol}$ scale at $23^{\circ} \mathrm{C}$ for $48 \mathrm{~h}$ using 10 mol\% 1 b (0.0286 g, $0.0498 \mathrm{mmol}), 3$ h $(0.175 \mathrm{~g}, 0.498 \mathrm{mmol}), \mathbf{2 o}(0.0977 \mathrm{~g}, 0.498$ $\mathrm{mmol})$, and diethyl ether $(1.2 \mathrm{~mL})$. The product was purified via flash chromatography on silica gel (10:90 hexanes:ethyl acetate) to provide $\mathbf{4 0}$ as a very viscous pale yellow oil $(0.210 \mathrm{~g}, 0.383 \mathrm{mmol}, 77 \%)$ in $96 \%$ ee. Chiral HPLC

(Chiralpak AS, 85:15 hexane:EtOH, $1.5 \mathrm{~mL} / \mathrm{min}, \lambda=208 \mathrm{~nm}) t_{\mathrm{R}}($ minor $)=11.8 \mathrm{~min}, t_{\mathrm{R}}($ major $)=34.4 \mathrm{~min}$; $[\alpha]^{25}{ }_{\mathrm{D}}=+23.7^{\circ}\left(\mathrm{c}=1.0, \mathrm{CHCl}_{3}\right)$; IR (thin film, $\left.\mathrm{cm}^{-1}\right) 3307(\mathrm{~m}), 3063(\mathrm{~m}), 2944(\mathrm{w}), 1531(\mathrm{~s}), 1452(\mathrm{~m})$, 1343 (s), 1249 (s), 1198 (m), 1086 (s), 1026 (s); ${ }^{1} \mathrm{H}-\mathrm{NMR}$ (500 MHz, CDCl ${ }_{3}$ ) 8 8.62-8.57 (2H, m, ArH), $8.13(1 \mathrm{H}, \mathrm{d}, J=8.3 \mathrm{~Hz}, \operatorname{ArH}), 8.06(1 \mathrm{H}, \mathrm{dd}, J=7.8,1.0 \mathrm{~Hz}, \operatorname{ArH}), 7.89-7.85(1 \mathrm{H}, \mathrm{m}, \operatorname{ArH}), 7.67-7.62(2 \mathrm{H}$, $\mathrm{m}, \operatorname{ArH}), 7.55-7.42(3 \mathrm{H}, \mathrm{m}, \operatorname{ArH}), 7.36-7.19(7 \mathrm{H}, \mathrm{m}, \operatorname{ArH}), 5.63\left(1 \mathrm{H}, \mathrm{dd}, J=15,7.8 \mathrm{~Hz}, \mathrm{O}-\mathrm{CH}_{2}\right), 5.55$ $\left(1 \mathrm{H}, \mathrm{dd}, J=15,6.8 \mathrm{~Hz}, \mathrm{O}-\mathbf{C H}_{2}\right), 5.40\left(1 \mathrm{H}, \mathrm{dd}, J=15,6.8 \mathrm{~Hz}, \mathrm{O}-\mathbf{C H}_{2}\right), 5.32(1 \mathrm{H}, \mathrm{dd}, J=15,7.8 \mathrm{~Hz}, \mathrm{O}-$ $\left.\mathrm{CH}_{2}\right), 4.24(1 \mathrm{H}, \mathrm{d}, J=21 \mathrm{~Hz}, \mathrm{P}-\mathrm{CH}), 3.85\left(1 \mathrm{H}, \mathrm{d}, J=13 \mathrm{~Hz}, \mathrm{~N}-\mathrm{CH}_{2}\right), 3.56\left(1 \mathrm{H}, \mathrm{d}, J=13 \mathrm{~Hz}, \mathrm{~N}-\mathbf{C H}_{2}\right)$, $2.41(1 \mathrm{H}, \mathrm{s}, \mathrm{N}-\mathrm{H}) ;{ }^{13} \mathrm{C}-\mathrm{NMR}\left(101 \mathrm{MHz}, \mathrm{CDCl}_{3}\right) \delta 150.3(\mathrm{~d}, J=7.8 \mathrm{~Hz}), 149.8(\mathrm{~d}, J=2.8 \mathrm{~Hz}), 146.8(\mathrm{~s})$, $146.6(\mathrm{~s}), 138.4(\mathrm{~s}), 135.9$ (d, $J=5.5 \mathrm{~Hz}), 134.1(\mathrm{~s}), 134.0(\mathrm{~s}), 132.6(\mathrm{~d}, J=6.9 \mathrm{~Hz}), 132.2(\mathrm{~d}, J=6.4 \mathrm{~Hz})$, $131.2(\mathrm{~d}, J=2.8 \mathrm{~Hz}), 128.9(\mathrm{~s}), 128.9(\mathrm{~s}), 128.6(\mathrm{~s}), 128.5(\mathrm{~s}), 128.4(\mathrm{~s}), 128.3(\mathrm{~s}), 127.5(\mathrm{~s}), 125.1(\mathrm{~s})$, $125.0(\mathrm{~s}), 123.9(\mathrm{~d}, J=2.3 \mathrm{~Hz}), 65.8(\mathrm{~d}, J=5.5 \mathrm{~Hz}), 65.0(\mathrm{~d}, J=6.0 \mathrm{~Hz}), 57.2(\mathrm{~d}, J=157 \mathrm{~Hz}), 51.2(\mathrm{~d}, J=$ $17 \mathrm{~Hz}$ ); ${ }^{31} \mathrm{P}-\mathrm{NMR}\left(162 \mathrm{MHz}, \mathrm{CDCl}_{3}\right) \delta 24.2$; HRMS (ES) $\mathrm{m} / \mathrm{z}$ for $\mathrm{C}_{27} \mathrm{H}_{26} \mathrm{~N}_{4} \mathrm{O}_{7} \mathrm{P}[\mathrm{M}+\mathrm{H}]^{+}$cacld. 549.1539, found 549.1533 .

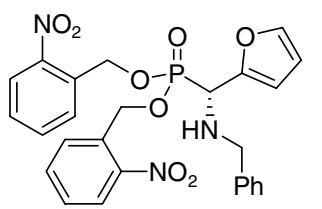

4p: Reaction was performed on a $0.50 \mathrm{mmol}$ scale at $4{ }^{\circ} \mathrm{C}$ for $72 \mathrm{~h}$ using $10 \mathrm{~mol} \%$ 1b $(0.0288 \mathrm{~g}, 0.0500 \mathrm{mmol}), 3 \mathrm{~h}(0.176 \mathrm{~g}, 0.500 \mathrm{mmol}), 2 \mathbf{p}(0.0926 \mathrm{~g}, 0.500 \mathrm{mmol})$, and diethyl ether $(1.2 \mathrm{~mL})$. The product was purified via flash chromatography on silica gel (60:40 hexanes:ethyl acetate) to provide $\mathbf{4} \mathbf{p}$ as a very viscous pale yellow oil $(0.239 \mathrm{~g}, 0.445 \mathrm{mmol}, 89 \%)$ in 92\% ee. Chiral HPLC (Chiralpak AD, 70:30

hexane:IPA, $1.0 \mathrm{~mL} / \mathrm{min}, \lambda=208 \mathrm{~nm}) t_{\mathrm{R}}($ major $)=41.2 \mathrm{~min}, t_{\mathrm{R}}($ minor $)=46.2 \mathrm{~min} ;[\alpha]^{25}{ }_{\mathrm{D}}=+35.0^{\circ}(\mathrm{c}=1.0$, $\mathrm{CHCl}_{3}$ ); IR (thin film, $\mathrm{cm}^{-1}$ ) 3326 (w), 3065 (w), 2944 (w), 1530 (s), 1449 (m), 1343 (s), 1252 (s), 1197 $(\mathrm{m}), 1086(\mathrm{~s}), 1025$ (s); ${ }^{1} \mathrm{H}-\mathrm{NMR}\left(500 \mathrm{MHz}, \mathrm{CDCl}_{3}\right) \delta 8.14(1 \mathrm{H}, \mathrm{dd}, J=8.3,1.0 \mathrm{~Hz}, \mathrm{ArH}), 8.09(1 \mathrm{H}, \mathrm{dd}, J$ $=7.8,1.0 \mathrm{~Hz}, \operatorname{ArH}), 7.77-7.73(1 \mathrm{H}, \mathrm{m}, \operatorname{ArH}), 7.67-7.63(1 \mathrm{H}, \mathrm{m}, \operatorname{ArH}), 7.61-7.43(5 \mathrm{H}, \mathrm{m}, \operatorname{ArH}), 7.30-7.22$ $(5 \mathrm{H}, \mathrm{m}, \mathrm{ArH}), 6.44-6.40(2 \mathrm{H}, \mathrm{m}, \mathrm{ArH}), 5.69\left(1 \mathrm{H}, \mathrm{dd}, J=15,7.8 \mathrm{~Hz}, \mathrm{O}-\mathrm{CH}_{2}\right), 5.58(1 \mathrm{H}, \mathrm{dd}, J=15,6.8 \mathrm{~Hz}$, $\left.\mathrm{O}-\mathrm{CH}_{2}\right), 5.47\left(1 \mathrm{H}, \mathrm{dd}, J=15,6.8 \mathrm{~Hz}, \mathrm{O}-\mathrm{CH}_{2}\right), 5.36\left(1 \mathrm{H}, \mathrm{dd}, J=15,7.8 \mathrm{~Hz}, \mathrm{O}-\mathrm{CH}_{2}\right), 4.29(1 \mathrm{H}, \mathrm{d}, J=23$ $\mathrm{Hz}, \mathrm{P}-\mathrm{CH}), 3.90\left(1 \mathrm{H}, \mathrm{d}, J=13 \mathrm{~Hz}, \mathrm{~N}-\mathrm{CH}_{2}\right), 3.62\left(1 \mathrm{H}, \mathrm{d}, J=13 \mathrm{~Hz}, \mathrm{~N}-\mathrm{CH}_{2}\right), 2.31(1 \mathrm{H}, \mathrm{s}, \mathrm{N}-\mathrm{H}) ;{ }^{13} \mathrm{C}-\mathrm{NMR}$ $\left(101 \mathrm{MHz}, \mathrm{CDCl}_{3}\right) \delta 148.9(\mathrm{~d}, J=2.3 \mathrm{~Hz}), 146.6(\mathrm{~s}), 146.5(\mathrm{~s}), 143.1(\mathrm{~d}, J=3.2 \mathrm{~Hz}), 138.6(\mathrm{~s}), 134.1(\mathrm{~s})$, $134.1(\mathrm{~s}), 133.0(\mathrm{~d}, J=6.9 \mathrm{~Hz}), 132.8(\mathrm{~d}, J=6.9 \mathrm{~Hz}), 128.7(\mathrm{~s}), 128.6(\mathrm{~s}), 128.4(\mathrm{~s}), 128.4(\mathrm{~s}), 128.4(\mathrm{~s})$, 128.2 (s), 127.3 (s), 124.9 (s), 124.9 (s), 110.8 (d, $J=2.3 \mathrm{~Hz}), 110.0$ (d, $J=8.2 \mathrm{~Hz}), 65.7$ (d, $J=5.5 \mathrm{~Hz})$, $65.0(\mathrm{~d}, J=5.5 \mathrm{~Hz}), 53.2(\mathrm{~d}, J=164 \mathrm{~Hz}), 51.3(\mathrm{~d}, J=17 \mathrm{~Hz}) ;{ }^{31} \mathrm{P}-\mathrm{NMR}\left(162 \mathrm{MHz}, \mathrm{CDCl}_{3}\right) \delta 22.3$; HRMS (ES) $\mathrm{m} / \mathrm{z}$ for $\mathrm{C}_{26} \mathrm{H}_{25} \mathrm{~N}_{3} \mathrm{O}_{8} \mathrm{P}[\mathrm{M}+\mathrm{H}]^{+}$cacld. 538.1397, found 538.1390. 


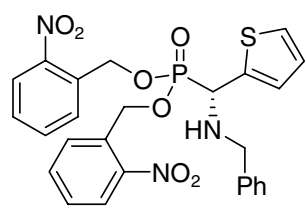

4q: Reaction was performed on a $0.50 \mathrm{mmol}$ scale at $23{ }^{\circ} \mathrm{C}$ for $48 \mathrm{~h}$ using $10 \mathrm{~mol} \%$ 1b $(0.0290 \mathrm{~g}, 0.0505 \mathrm{mmol}), 3 \mathbf{h}(0.178 \mathrm{~g}, 0.505 \mathrm{mmol}), 2 \mathbf{2 q}(0.102 \mathrm{~g}, 0.505 \mathrm{mmol})$, and diethyl ether $(1.2 \mathrm{~mL})$. The product was purified via flash chromatography on silica gel (60:40 hexanes:ethyl acetate) to provide $\mathbf{4 q}$ as a very viscous pale yellow oil $(0.249 \mathrm{~g}, 0.450 \mathrm{mmol}, 89 \%)$ in 94\% ee. Chiral HPLC (Chiralcel OD, 95:05

hexane:EtOH, $1.0 \mathrm{~mL} / \mathrm{min}, \lambda=208 \mathrm{~nm}) t_{\mathrm{R}}($ minor $)=44.8 \mathrm{~min}, t_{\mathrm{R}}$ (major) $=50.6 \mathrm{~min} ;[\alpha]^{25}{ }_{\mathrm{D}}=+17.4^{\circ}(\mathrm{c}=$ 1.2, $\mathrm{CHCl}_{3}$ ); IR (thin film, cm $\left.{ }^{-1}\right) 3314(\mathrm{w}), 3065$ (m), $2853(\mathrm{~m}), 1528$ (s), 1448 (s), 1342 (s), 1251 (s), 1196 (m), 1086 (s), 1023 (s); ${ }^{1} \mathrm{H}-\mathrm{NMR}\left(500 \mathrm{MHz}, \mathrm{CDCl}_{3}\right) \delta 8.13(1 \mathrm{H}, \mathrm{dd}, J=8.3,1.0 \mathrm{~Hz}, \mathrm{ArH}), 8.07(1 \mathrm{H}, \mathrm{dd}, J$ $=8.3,1.2 \mathrm{~Hz}, \operatorname{ArH}), 7.73-7.70(1 \mathrm{H}, \mathrm{m}, \operatorname{ArH}), 7.67-7.63(1 \mathrm{H}, \mathrm{m}, \operatorname{ArH}), 7.57-7.42(4 \mathrm{H}, \mathrm{m}, \operatorname{ArH}), 7.35-7.24$ $(6 \mathrm{H}, \mathrm{m}, \mathrm{ArH}), 7.16-7.13(1 \mathrm{H}, \mathrm{m}, \mathrm{ArH}), 7.06-7.03(1 \mathrm{H}, \mathrm{m}, \mathrm{ArH}), 5.64\left(1 \mathrm{H}, \mathrm{dd}, J=15,7.8 \mathrm{~Hz}, \mathrm{O}-\mathrm{CH}_{2}\right)$, $5.57\left(1 \mathrm{H}, \mathrm{dd}, J=15,6.8 \mathrm{~Hz}, \mathrm{O}-\mathrm{CH}_{2}\right), 5.43\left(1 \mathrm{H}, \mathrm{dd}, J=15,6.8 \mathrm{~Hz}, \mathrm{O}-\mathrm{CH}_{2}\right), 5.33(1 \mathrm{H}, \mathrm{dd}, J=15,7.8 \mathrm{~Hz}$, $\left.\mathrm{O}-\mathrm{CH}_{2}\right), 4.47(1 \mathrm{H}, \mathrm{d}, J=21 \mathrm{~Hz}, \mathrm{P}-\mathrm{CH}), 3.95\left(1 \mathrm{H}, \mathrm{d}, J=13 \mathrm{~Hz}, \mathrm{~N}-\mathrm{CH}_{2}\right), 3.67\left(1 \mathrm{H}, \mathrm{d}, J=13 \mathrm{~Hz}, \mathrm{~N}-\mathrm{C} \mathbf{H}_{2}\right)$, $2.30(1 \mathrm{H}, \mathrm{s}, \mathrm{N}-\mathrm{H}) ;{ }^{13} \mathrm{C}-\mathrm{NMR}\left(101 \mathrm{MHz}, \mathrm{CDCl}_{3}\right) \delta 146.6(\mathrm{~s}), 146.5(\mathrm{~s}), 138.7(\mathrm{~s}), 138.5(\mathrm{~d}, J=2.7 \mathrm{~Hz})$, $134.1(\mathrm{~s}), 134.0$ (s), 132.9 (d, $J=6.9 \mathrm{~Hz}), 132.1(\mathrm{~d}, J=6.4 \mathrm{~Hz}), 128.7(\mathrm{~s}), 128.6(\mathrm{~s}), 128.5(\mathrm{~s}), 128.5(\mathrm{~s})$, $128.5(\mathrm{~s}), 128.3(\mathrm{~s}), 127.5(\mathrm{~d}, J=8.2 \mathrm{~Hz}), 127.4(\mathrm{~s}), 127.2(\mathrm{~d}, J=2.7 \mathrm{~Hz}), 126.1(\mathrm{~d}, J=3.7 \mathrm{~Hz}), 124.9(\mathrm{~s})$, $124.9(\mathrm{~s}), 65.8(\mathrm{~d}, J=6.0 \mathrm{~Hz}), 65.1(\mathrm{~d}, J=6.0 \mathrm{~Hz}), 54.8(\mathrm{~d}, J=164 \mathrm{~Hz}), 51.1(\mathrm{~d}, J=17 \mathrm{~Hz}) ;{ }^{31} \mathrm{P}-\mathrm{NMR}$ $\left(162 \mathrm{MHz}, \mathrm{CDCl}_{3}\right) \delta 23.2 ; \mathrm{HRMS}(\mathrm{ES}) \mathrm{m} / \mathrm{z}$ for $\mathrm{C}_{26} \mathrm{H}_{25} \mathrm{~N}_{3} \mathrm{O}_{7} \mathrm{PS}[\mathrm{M}+\mathrm{H}]^{+}$cacld. 554.1151, found 554.1160.

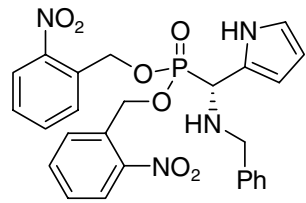

4r: Reaction was performed on a $0.50 \mathrm{mmol}$ scale at $4{ }^{\circ} \mathrm{C}$ for $48 \mathrm{~h}$ using $10 \mathrm{~mol} \%$ 1b $(0.0287 \mathrm{~g}, 0.0500 \mathrm{mmol}), 3 \mathbf{h}(0.176 \mathrm{~g}, 0.500 \mathrm{mmol}), 2 \mathbf{r}(0.0921 \mathrm{~g}, 0.500 \mathrm{mmol})$, and diethyl ether $(1.2 \mathrm{~mL})$. The product was purified via flash chromatography on silica gel (60:40 hexanes:ethyl acetate) to provide $4 \mathbf{r}$ as a very viscous pale yellow oil $(0.231 \mathrm{~g}, 0.431 \mathrm{mmol}, 86 \%)$ in 81\% ee. Chiral HPLC (Chiralpak AD, 70:30

hexane:IPA, $1.0 \mathrm{~mL} / \mathrm{min}, \lambda=208 \mathrm{~nm}) t_{\mathrm{R}}($ minor $)=18.1 \mathrm{~min}, t_{\mathrm{R}}($ major $)=21.8 \mathrm{~min} ;[\alpha]_{\mathrm{D}}^{25}=+27.8^{\circ}(\mathrm{c}=1.0$, $\mathrm{CHCl}_{3}$ ); IR (thin film, $\mathrm{cm}^{-1}$ ) 3405 (m), 3300 (s), 3065 (m), 2944 (m), 1530 (s), 1449 (m), 1343 (s), 1229 (s), $1198(\mathrm{~m}), 1086$ (s), 1026 (s); ${ }^{1} \mathrm{H}-\mathrm{NMR}\left(500 \mathrm{MHz}, \mathrm{CDCl}_{3}\right) \delta 9.14(1 \mathrm{H}, \mathrm{brs}, \mathrm{N}-\mathrm{H}), 8.14(1 \mathrm{H}, \mathrm{d}, J=7.8 \mathrm{~Hz}$, ArH), $8.06(1 \mathrm{H}, \mathrm{dd}, J=8.3,1.0 \mathrm{~Hz}, \mathrm{ArH}), 7.73-7.64(2 \mathrm{H}, \mathrm{m}, \mathrm{ArH}), 7.58-7.41(3 \mathrm{H}, \mathrm{m}, \mathrm{ArH}), 7.32-7.20$ $(6 \mathrm{H}, \mathrm{m}, \mathrm{ArH}), 6.83-6.78(1 \mathrm{H}, \mathrm{m}, \mathrm{ArH}), 6.18-6.14(2 \mathrm{H}, \mathrm{m}, \mathrm{ArH}), 5.63\left(1 \mathrm{H}, \mathrm{dd}, J=15,7.8 \mathrm{~Hz}, \mathrm{O}-\mathrm{CH}_{2}\right)$, $5.52\left(1 \mathrm{H}, \mathrm{dd}, J=15,6.8 \mathrm{~Hz}, \mathrm{O}-\mathrm{CH}_{2}\right), 5.34\left(1 \mathrm{H}, \mathrm{dd}, J=15,7.3 \mathrm{~Hz}, \mathrm{O}-\mathrm{CH}_{2}\right), 5.20(1 \mathrm{H}, \mathrm{dd}, J=15,7.8 \mathrm{~Hz}$, O-CH 2$), 4.26(1 \mathrm{H}, \mathrm{d}, J=21 \mathrm{~Hz}, \mathrm{P}-\mathrm{CH}), 3.86\left(1 \mathrm{H}, \mathrm{d}, J=13 \mathrm{~Hz}, \mathrm{~N}-\mathrm{CH}_{2}\right), 3.63\left(1 \mathrm{H}, \mathrm{d}, J=13 \mathrm{~Hz}, \mathrm{~N}-\mathrm{C} \mathbf{H}_{2}\right)$, $2.27\left(1 \mathrm{H}\right.$, brs, N-H) ${ }^{13} \mathrm{C}-\mathrm{NMR}\left(101 \mathrm{MHz}, \mathrm{CDCl}_{3}\right) \delta 146.7(\mathrm{~s}), 146.5(\mathrm{~s}), 139.2(\mathrm{~s}), 134.3(\mathrm{~s}), 134.2(\mathrm{~s})$, $133.1(\mathrm{~d}, J=7.3 \mathrm{~Hz}), 133.0(\mathrm{~d}, J=6.4 \mathrm{~Hz}), 128.8(\mathrm{~s}), 128.6(\mathrm{~s}), 128.6(\mathrm{~s}), 128.4(\mathrm{~s}), 128.4(\mathrm{~s}), 128.3(\mathrm{~s})$, $127.2(\mathrm{~s}), 125.0(\mathrm{~s}), 124.9(\mathrm{~s}), 124.3(\mathrm{~d}, J=4.6 \mathrm{~Hz}), 119.4(\mathrm{~s}), 110.2(\mathrm{~d}, J=10.2 \mathrm{~Hz}), 108.3(\mathrm{~s}), 65.9(\mathrm{~d}, J=$ $5.5 \mathrm{~Hz}), 65.8(\mathrm{~d}, J=5.5 \mathrm{~Hz}), 52.9(\mathrm{~d}, J=162 \mathrm{~Hz}), 51.1(\mathrm{~d}, J=18 \mathrm{~Hz}) ;{ }^{31} \mathrm{P}-\mathrm{NMR}\left(162 \mathrm{MHz}, \mathrm{CDCl}_{3}\right) \delta$ 24.6; HRMS (ES) $\mathrm{m} / \mathrm{z}$ for $\mathrm{C}_{26} \mathrm{H}_{26} \mathrm{~N}_{4} \mathrm{O}_{7} \mathrm{P}[\mathrm{M}+\mathrm{H}]^{+}$cacld. 537.1539, found 537.1536.

\section{Deprotection.}
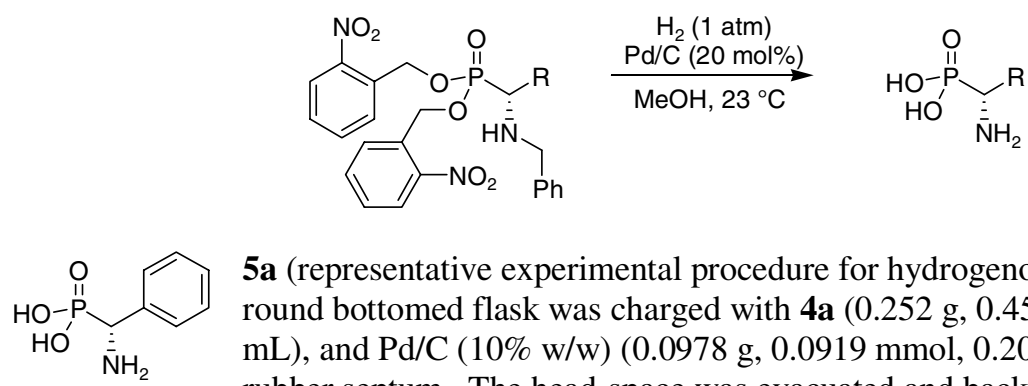

$5 a$ (representative experimental procedure for hydrogenolytic deprotection): A 100-mL round bottomed flask was charged with $4 \mathrm{a}(0.252 \mathrm{~g}, 0.459 \mathrm{mmol}, 98 \% \mathrm{ee})$, methanol (7.5 $\mathrm{mL})$, and $\mathrm{Pd} / \mathrm{C}(10 \% \mathrm{w} / \mathrm{w})(0.0978 \mathrm{~g}, 0.0919 \mathrm{mmol}, 0.20$ equivalents $)$ and sealed with a rubber septum. The head-space was evacuated and back-filled with nitrogen. The headspace was evacuated and back-filled with hydrogen three times and then stirred under a balloon of hydrogen for $24 \mathrm{~h}$. The reaction mixture was concentrated in vacuo. The crude solid was suspended in ethyl acetate $(5 \mathrm{~mL})$ and vacuum filtered. The light gray solid residue was washed with ethyl acetate (65 $\mathrm{mL})$. The collection flask was replaced, and the solid was washed with deionized water $(70 \mathrm{~mL})$. The aqueous solution was vacuum filtered a second time to remove any trace $\mathrm{Pd} / \mathrm{C}$ and then concentrated in vacuo to provide the product $5 \mathbf{a}$ as a white solid $(0.0745 \mathrm{~g}, 0.398 \mathrm{mmol}, 87 \%)$ in $97 \%$ ee. Chiral HPLC (Chiralcel OD, 95:05 hexane:IPA, $1.0 \mathrm{~mL} / \mathrm{min}, \lambda=208 \mathrm{~nm}$ ) $t_{\mathrm{R}}($ minor $)=19.2 \mathrm{~min}, t_{\mathrm{R}}($ major $)=30.2 \mathrm{~min}$ (as 
$N$-Cbz dimethyl phosphonate derivative $) ;[\alpha]^{23}{ }_{\mathrm{D}}=+17.1^{\circ}\left(\mathrm{c}=0.96,1 \mathrm{~N} \mathrm{NaOH}_{\mathrm{aq}}\right)\left(\mathrm{lit}^{5}{ }^{5}[\alpha]^{20}{ }_{\mathrm{D}}=+18.2(\mathrm{c}=\right.$ 0.7, $\left.1 \mathrm{~N} \mathrm{NaOH}_{\mathrm{aq}}\right)$ ); $\mathrm{mp}=280-283^{\circ} \mathrm{C}\left(\mathrm{lit}^{5} .^{5}=284-285^{\circ} \mathrm{C}\right) ; \mathrm{IR}\left(\mathrm{KBr}, \mathrm{cm}^{-1}\right) 3219(\mathrm{~m}), 3003(\mathrm{~s}), 2922(\mathrm{~s}), 1620$ (m), 1539 (m), 1267 (m), 1188 (s), 1067 (s), 916 (s); ${ }^{1} \mathrm{H}-\mathrm{NMR}\left(500 \mathrm{MHz}, 0.5 \mathrm{M} \mathrm{NaOD}_{\mathrm{aq}}\right.$ ) $\delta$ 7.34-7.17 (5H, $\mathrm{m}, \mathrm{ArH}), 3.71(1 \mathrm{H}, \mathrm{d}, J=16 \mathrm{~Hz}, \mathrm{P}-\mathrm{CH}) ;{ }^{13} \mathrm{C}-\mathrm{NMR}\left(126 \mathrm{MHz}, 0.5 \mathrm{M} \mathrm{NaOD}_{\mathrm{aq}}\right) \delta 145.5(\mathrm{~s}), 128.6(\mathrm{~s}), 128.2$ (s), $126.8(\mathrm{~s}), 56.1(\mathrm{~d}, J=131 \mathrm{~Hz}) ;{ }^{31} \mathrm{P}-\mathrm{NMR}\left(162 \mathrm{MHz}, \mathrm{CDCl}_{3}\right) \delta 18.7$; HRMS (ES) $\mathrm{m} / \mathrm{z}$ for $\mathrm{C}_{7} \mathrm{H}_{11} \mathrm{NO}_{3} \mathrm{P}$ $[\mathrm{M}+\mathrm{H}]^{+}$calcd. 188.0476, found 188.0483.

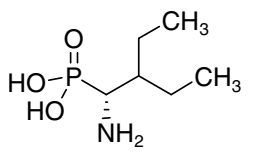

5b: Reaction was performed on a $0.42 \mathrm{mmol}$ scale using $\mathbf{4 b}(0.227 \mathrm{~g}, 0.419 \mathrm{mmol}, 96 \%$ $e e)$, methanol $(7.0 \mathrm{~mL})$, and $\mathrm{Pd} / \mathrm{C}(10 \% \mathrm{w} / \mathrm{w})(0.0893 \mathrm{~g}, 0.0838 \mathrm{mmol}, 0.20$ equiv) for $24 \mathrm{~h}$. The aqueous washes were concentrated in vacuo to provide the product $\mathbf{5 b}$ as a white solid (0.0678 g, $0.374 \mathrm{mmol}, 89 \%)$ in 96\% ee. Chiral HPLC (Chiralcel OD, 95:05

hexane:IPA, $1.0 \mathrm{~mL} / \mathrm{min}, \lambda=208 \mathrm{~nm}) t_{\mathrm{R}}($ minor $)=9.9 \mathrm{~min}, t_{\mathrm{R}}$ (major) $=14.4 \mathrm{~min}($ as $N$-Cbz dimethyl phosphonate derivative $) ;[\alpha]^{24}=-15.5^{\circ}\left(\mathrm{c}=0.78,1 \mathrm{~N} \mathrm{NaOH}_{\mathrm{aq}}\right) ; \mathrm{mp}=263-266^{\circ} \mathrm{C} ; \mathrm{IR}\left(\mathrm{KBr}, \mathrm{cm}^{-1}\right) 3133(\mathrm{~s})$, 3092 (s), 2969 (s), 2880 (s), 2683 (w), 1526 (m), 1217 (s), 1165 (w), 1059 (s), 1026 (m), 932 (m); ${ }^{1} \mathrm{H}-\mathrm{NMR}$ $\left(500 \mathrm{MHz}, 0.5 \mathrm{M} \mathrm{NaOD}_{\mathrm{aq}}\right) \delta 2.60(1 \mathrm{H}, \mathrm{dd}, J=14,2.4 \mathrm{~Hz}, \mathrm{P}-\mathrm{CH}), 1.71-1.61\left(1 \mathrm{H}, \mathrm{m}, \mathrm{CH}\left(\mathrm{CH}_{2} \mathrm{CH}_{3}\right)_{2}\right), 1.48-$ $1.36\left(2 \mathrm{H}, \mathrm{m}, \mathrm{CH}_{2} \mathrm{CH}_{3}\right), 1.28-1.16\left(1 \mathrm{H}, \mathrm{m}, \mathrm{CH}_{2} \mathrm{CH}_{3}\right), 1.05-0.94\left(1 \mathrm{H}, \mathrm{m}, \mathrm{CH}_{2} \mathrm{CH}_{3}\right), 0.79(3 \mathrm{H}, \mathrm{dd}, J=8.5,6.9$ $\left.\mathrm{Hz}, \mathrm{CH}_{3}\right), 0.78\left(3 \mathrm{H}, \mathrm{dd}, J=7.3,6.1 \mathrm{~Hz}, \mathrm{CH}_{3}\right) ;{ }^{13} \mathrm{C}-\mathrm{NMR}\left(126 \mathrm{MHz}, 0.5 \mathrm{M} \mathrm{NaOD}_{\mathrm{aq}}\right) \delta 51.6(\mathrm{~d}, J=138$ $\mathrm{Hz}), 42.5(\mathrm{~s}), 23.3(\mathrm{~d}, J=10 \mathrm{~Hz}), 21.8(\mathrm{~s}), 12.1(\mathrm{~s}), 11.9(\mathrm{~s}) ;{ }^{31} \mathrm{P}-\mathrm{NMR}\left(162 \mathrm{MHz}, \mathrm{CDCl}_{3}\right) \delta 22.7 ; \mathrm{HRMS}$ (ES) $\mathrm{m} / \mathrm{z}$ for $\mathrm{C}_{6} \mathrm{H}_{17} \mathrm{NO}_{3} \mathrm{P}[\mathrm{M}+\mathrm{H}]^{+}$calcd. 182.0946, found 182.0941 .

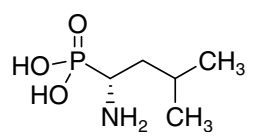

5f $(R)$-Leu ${ }^{\mathrm{P}}$ : Reaction was performed on a $1.31 \mathrm{mmol}$ scale using $4 \mathbf{f}(0.689 \mathrm{~g}, 1.31$ $\mathrm{mmol}, 99 \%$ ee $)$, methanol $(20 \mathrm{~mL})$, and $\mathrm{Pd} / \mathrm{C}(10 \% \mathrm{w} / \mathrm{w})(0.279 \mathrm{~g}, 0.262 \mathrm{mmol}, 0.20$ equiv) for $72 \mathrm{~h}$. The aqueous washes were concentrated in vacuo to provide the product 5f as a white solid (0.204 g, $1.22 \mathrm{mmol}, 93 \%)$ in 98\% ee. Chiral HPLC (Chiralcel OD, 95:05 hexane:EtOH, $1.0 \mathrm{~mL} / \mathrm{min}, \lambda=208 \mathrm{~nm}) t_{\mathrm{R}}($ minor $)=10.7 \mathrm{~min}, t_{\mathrm{R}}$ (major) $=12.7 \mathrm{~min}$ (as $N$-Cbz dimethyl phosphonate derivative); $[\alpha]^{24}{ }_{\mathrm{D}}=-29.0^{\circ}\left(\mathrm{c}=1.0,1 \mathrm{~N} \mathrm{NaOH}_{\mathrm{aq}}\right)\left(\mathrm{lit}^{6}{ }^{6}[\alpha]^{25}{ }_{\mathrm{D}}=-29.5\left(\mathrm{c}=1,1 \mathrm{~N} \mathrm{NaOH}_{\mathrm{aq}}\right)\right) ; \mathrm{mp}$ $=280-282^{\circ} \mathrm{C}\left(\right.$ lit. $\left.^{6} \mathrm{mp}=285-286^{\circ} \mathrm{C}\right) ; \mathrm{IR}\left(\mathrm{KBr}, \mathrm{cm}^{-1}\right) 3084(\mathrm{~m}), 2961(\mathrm{~s}), 2872(\mathrm{~s}), 1535(\mathrm{~s}), 1248(\mathrm{~m}), 1180$ (s), 1028 (s), 926 (s); ${ }^{1} \mathrm{H}-\mathrm{NMR}\left(500 \mathrm{MHz}, 0.5 \mathrm{M} \mathrm{NaOD}_{\mathrm{aq}}\right) \delta 2.52(1 \mathrm{H}, \mathrm{dt}, J=11,2.9 \mathrm{~Hz}, \mathrm{P}-\mathrm{CH}), 1.72-1.61$ $\left(1 \mathrm{H}, \mathrm{m}, \mathrm{CH}\left(\mathrm{CH}_{3}\right)_{2}\right), 1.41-1.30\left(1 \mathrm{H}, \mathrm{m}, \mathrm{CH}_{2}\right), 1.29-1.19\left(1 \mathrm{H}, \mathrm{m}, \mathrm{CH}_{2}\right), 0.83\left(3 \mathrm{H}, \mathrm{d}, J=6.8 \mathrm{~Hz}, \mathrm{CH}_{3}\right), 0.77$ $\left(3 \mathrm{H}, \mathrm{d}, J=6.3 \mathrm{~Hz}, \mathrm{CH}_{3}\right) ;{ }^{13} \mathrm{C}-\mathrm{NMR}\left(126 \mathrm{MHz}, 0.5 \mathrm{M} \mathrm{NaOD}_{\text {aq }}\right) \delta 48.3(\mathrm{~d}, J=138 \mathrm{~Hz}), 41.4(\mathrm{~s}), 24.8(\mathrm{~d}, J=$ $13 \mathrm{~Hz}$ ), $24.1(\mathrm{~s}), 21.0(\mathrm{~s}) ;{ }^{31} \mathrm{P}-\mathrm{NMR}\left(162 \mathrm{MHz}, \mathrm{CDCl}_{3}\right) \delta 23.0$; HRMS (ES) $\mathrm{m} / \mathrm{z}$ for $\mathrm{C}_{10} \mathrm{H}_{29} \mathrm{~N}_{2} \mathrm{O}_{6} \mathrm{P}_{2}$ $[2 \mathrm{M}+\mathrm{H}]^{+}$calcd. 335.1501 , found 335.1505 .

\section{General Procedure for Generating $N$-Cbz Dimethyl Phosphonate Derivative for \%ee Determination by Chiral HPLC.}
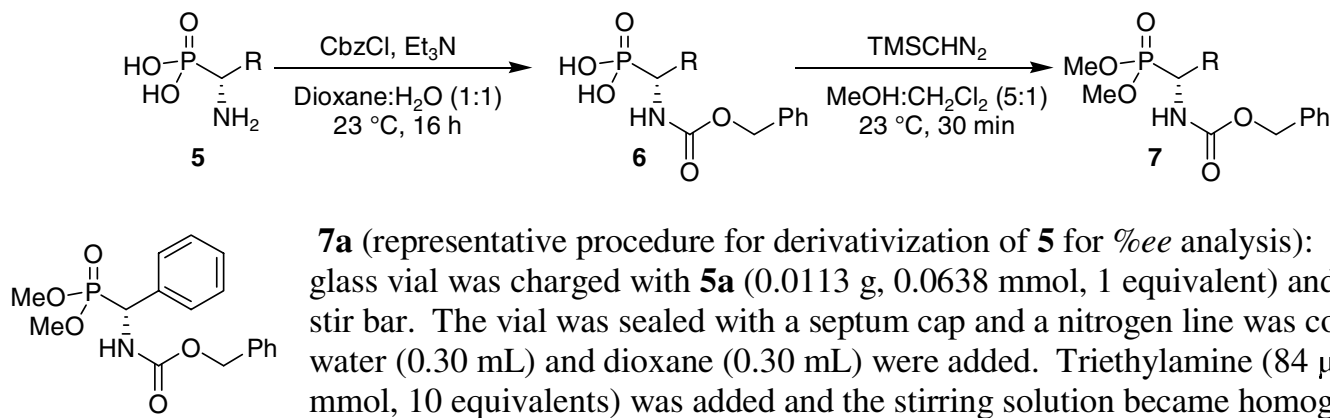

7a (representative procedure for derivativization of 5 for \%ee analysis): A 4-mL glass vial was charged with $\mathbf{5 a}(0.0113 \mathrm{~g}, 0.0638 \mathrm{mmol}, 1$ equivalent $)$ and a magnetic stir bar. The vial was sealed with a septum cap and a nitrogen line was connected. DI water $(0.30 \mathrm{~mL})$ and dioxane $(0.30 \mathrm{~mL})$ were added. Triethylamine $(84 \mu \mathrm{L}, 0.603$ mmol, 10 equivalents) was added and the stirring solution became homogeneous.

Finally, benzyl chloroformate ( $43 \mu \mathrm{L}, 0.302 \mathrm{mmol}, 5$ equivalents) was added drop-wise. After $16 \mathrm{~h}$, the reaction mixture was transferred to a $30 \mathrm{~mL}$ separatory funnel with a saturated aqueous solution of sodium carbonate ( $3 \mathrm{~mL}$, to litmus blue) and washed with diethyl ether $(2 \mathrm{X} 7 \mathrm{~mL})$. The aqueous solution was acidified to $\mathrm{pH}<1$ (litmus red) by the drop-wise addition of a concentrated solution of hydrochloric acid. The phosphonic acid precipitated out of solution and was extracted into ethyl acetate ( $3 \times 7 \mathrm{~mL})$. The combined ethyl acetate solution was dried over sodium sulfate, filtered, and concentrated in vacuo to yield crude $6 \mathbf{a}(0.0183 \mathrm{~g}, 0.0570 \mathrm{mmol}, 94 \%)$ as a white solid. This crude material was transferred to a 4-mL glass vial equipped with a magnetic stir bar, septum cap and nitrogen line. Methanol $(0.55 \mathrm{~mL})$ and 
dichloromethane $(0.10 \mathrm{~mL})$ were added. A $2.0 \mathrm{M}$ solution of (trimethylsilyl)diazomethane in hexanes $(0.17 \mathrm{~mL}, 0.34 \mathrm{mmol}, 6$ equivalents) was added drop-wise over 30 seconds. After 30 minutes, the reaction was concentrated in vacuo. The crude residue was purified by flash chromatography on silica gel (25:75 hexanes:ethyl acetate) to provide $7 \mathbf{a}(0.0079 \mathrm{~g}, 0.0226 \mathrm{mmol}, 40 \%, 37 \%$ from 5a $)$ as a white solid which was used directly for \%ee determination by chiral HPLC.

\section{References}

1. Wenzel, A.G.; Jacobsen, E.N. J. Am. Chem. Soc. 2002, 124, 12964-12965.

2. Vachal, P.V.; Jacobsen, E.N. J. Am. Chem. Soc. 2002, 124, 10012-10014.

3. Sigman, M.S.; Vachal, P.; Jacobsen, E.N. Angew. Chem. Int. Ed. 2000, 39, 1279-1281.

4. Fölsch, G. Acta. Chem. Scand. 1956, 10, 686.

5. Davis, F.A.; Lee, S.; Yan, H.; Titus, D.D. Org. Lett. 2001, 3, 1757-1760.

6. Giannousis, P.P.; Bartlett, P.A. J. Med. Chem. 1987, 30, 1603-1609. 


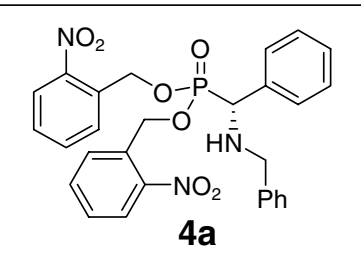

Chiral HPLC: Pirkle Covalent L-Leucine, 95:05 Hex:IPA, 1.0 ml/min, 208 nm

racemic

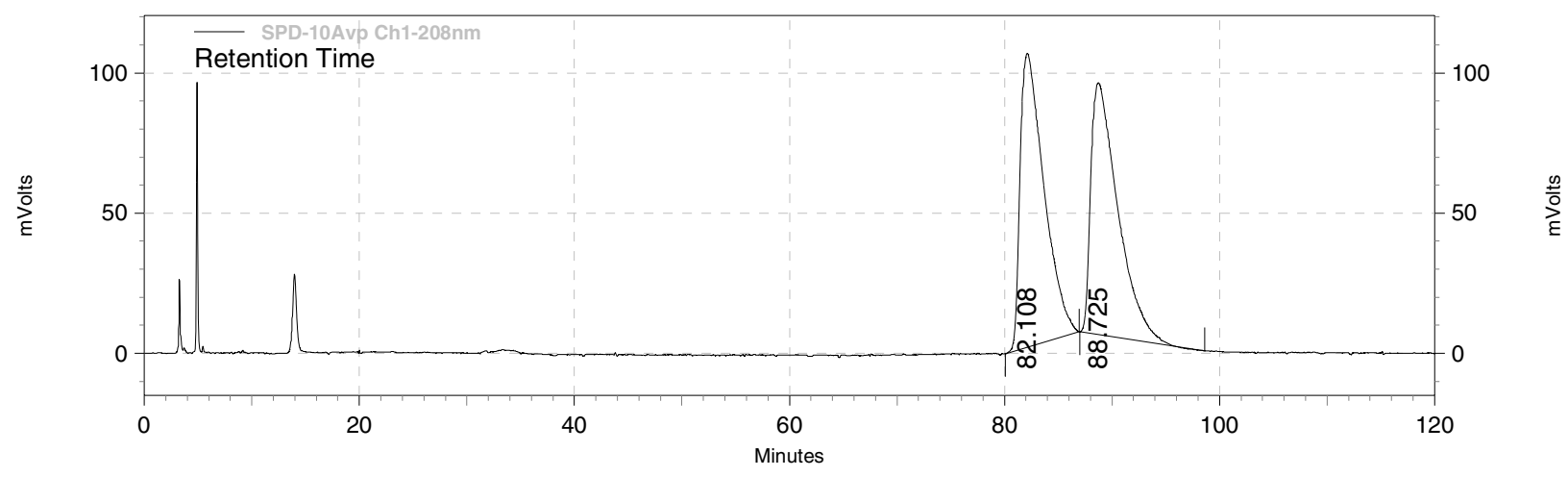

SPD-10Avp Ch1-208nm Results

Retention Time

Area

Area Percent

$\begin{array}{lll}82.11 & 16128328 & 50.66 \\ 88.72 & 15707217 & 49.34\end{array}$

\begin{tabular}{|r|r|r|}
\hline Totals & 31835545 & 100.00 \\
\hline
\end{tabular}

$98 \% e e$

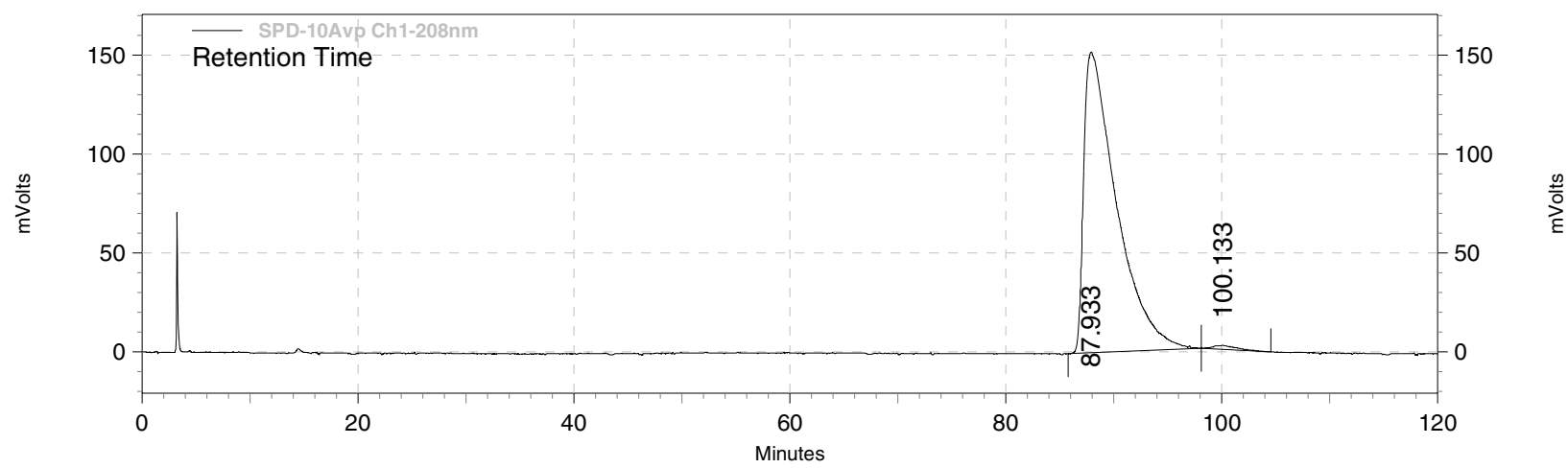

SPD-10Avp Ch1-208nm Results

Retention Time

87.93

Area

Area Percent

100.13

31992751

98.99

Totals

327830

1.01

\begin{tabular}{|r|r|r|}
\hline Totals & 32320581 & 100.00 \\
\hline
\end{tabular}




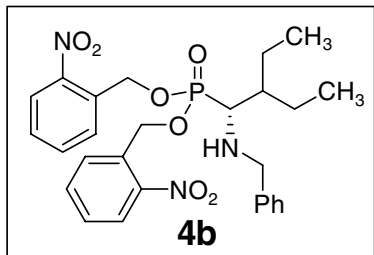

Chiral HPLC: Chiralcel OD, 97:03 Hex:EtOH, $1.0 \mathrm{ml} / \mathrm{min}, 208 \mathrm{~nm}$ racemic

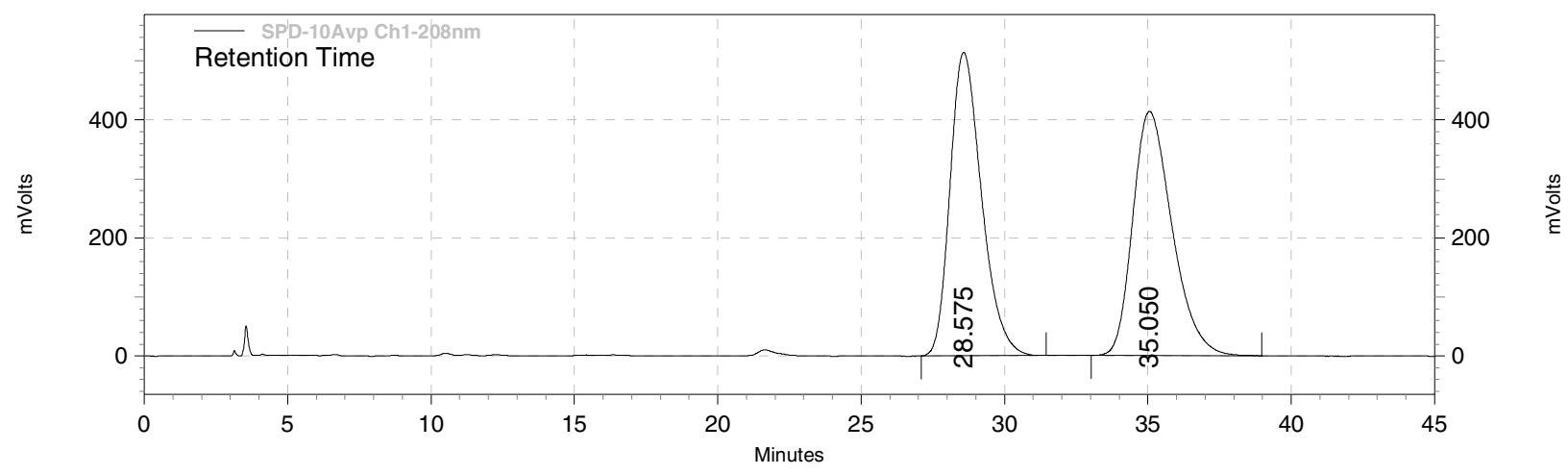

SPD-10Avp Ch1-208nm Results

Retention Time Area

Area Percent

$\begin{array}{lrr}28.57 & 39348849 & 49.89 \\ 35.05 & 39522276 & 50.11\end{array}$

\begin{tabular}{|r|r|r|}
\hline Totals & 78871125 & 100.00 \\
\hline
\end{tabular}

$96 \% e e$

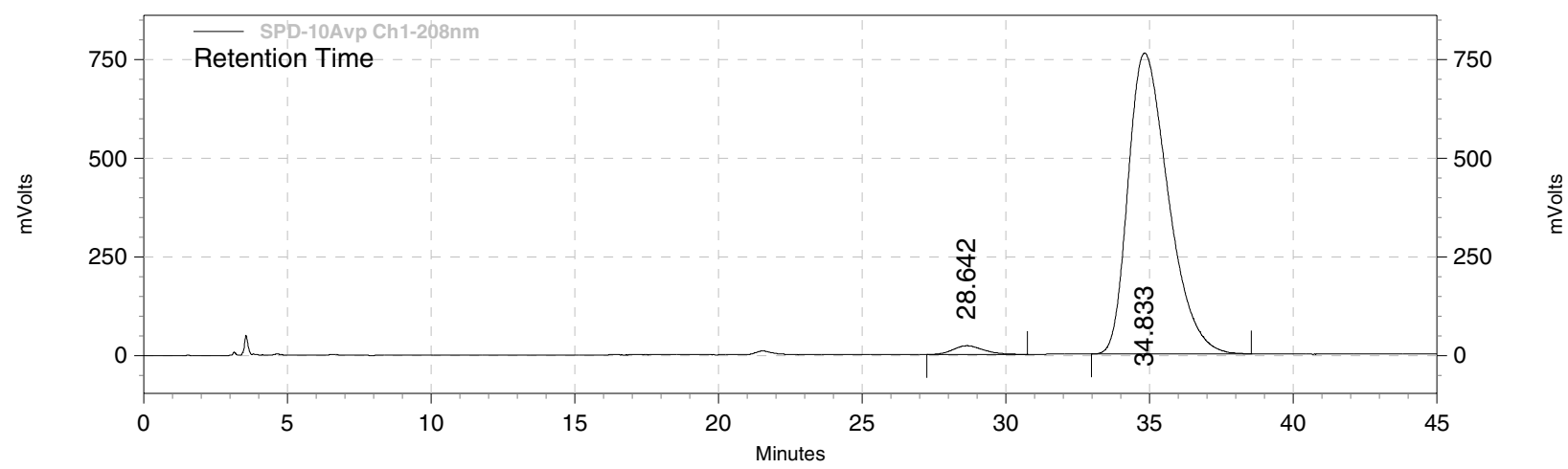

SPD-10Avp Ch1-208nm Results Retention Time 28.64 Area Area Percent 34.83 1594678 2.11 74151150 97.89

Totals 


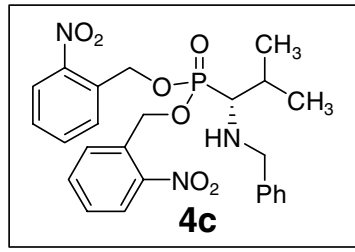

Chiral HPLC: Chiralcel OD, 97:03 Hex:EtOH, 1.0 ml/min, 208 nm

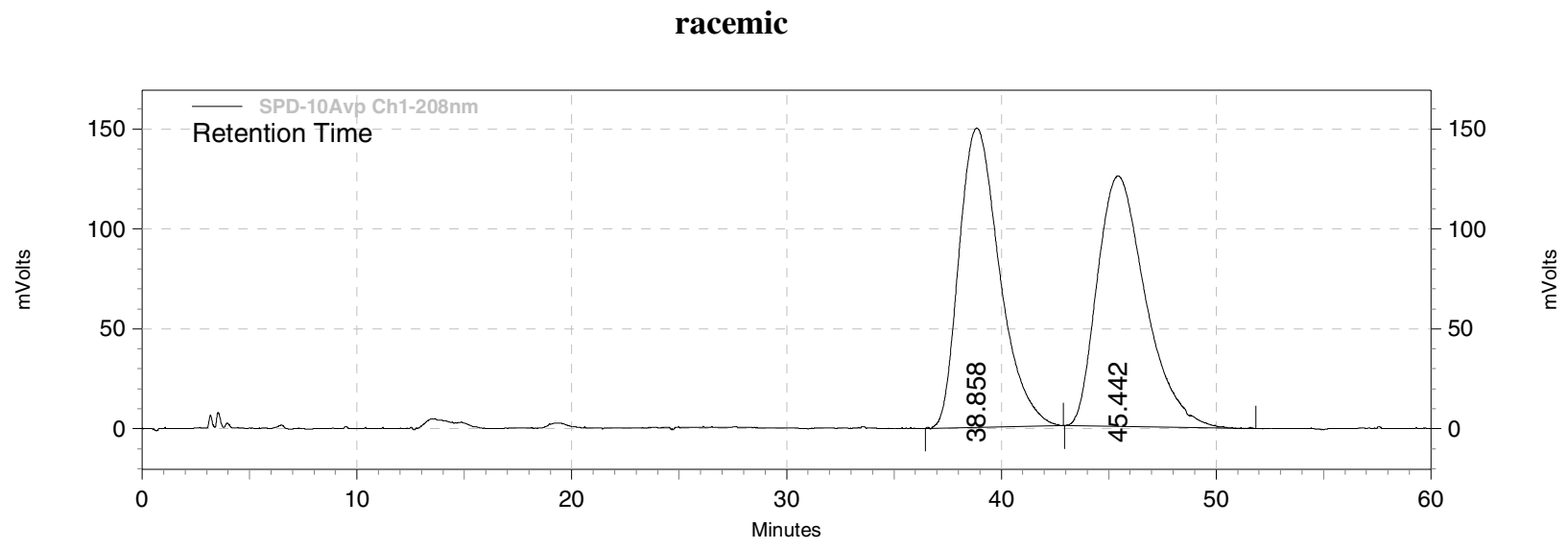

SPD-10Avp Ch1-208nm Results

Retention Time

Area

Area Percent

$\begin{array}{lll}38.86 & 19659064 & 50.05 \\ 45.44 & 19621581 & 49.95\end{array}$

\begin{tabular}{|r|r|r|}
\hline Totals & 39280645 & 100.00 \\
\hline
\end{tabular}

$90 \% e e$

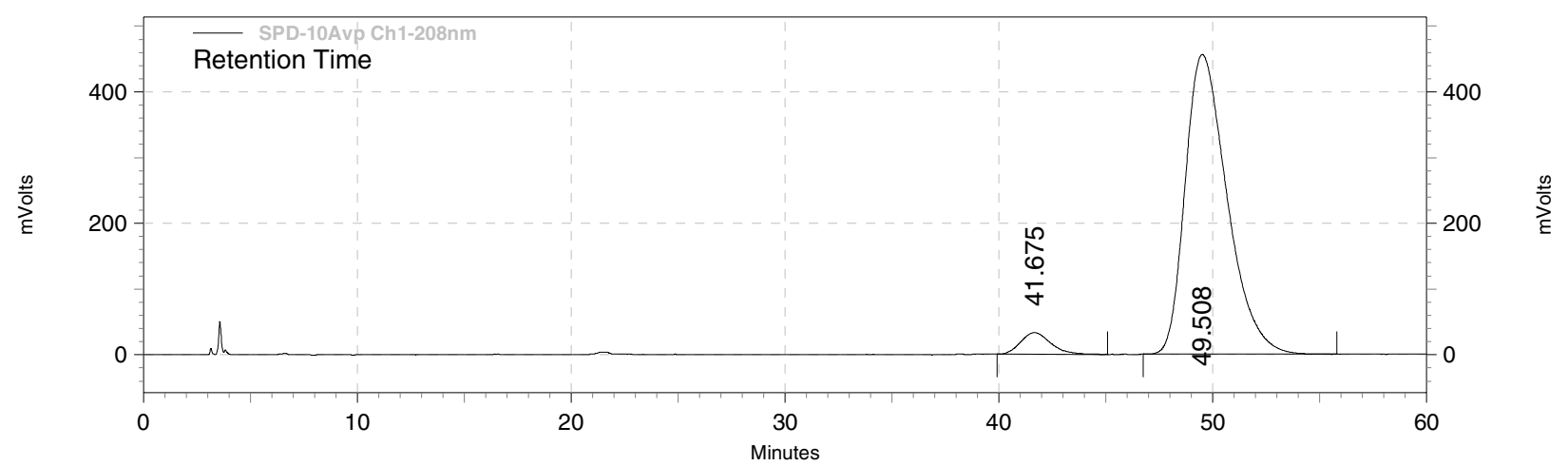

SPD-10Avp Ch1-208nm Results

\begin{tabular}{r|r|r|}
\multicolumn{1}{r}{ Retention Time } & Area & Area Percent \\
\hline 41.67 & 3236470 & 4.95 \\
49.51 & 62143407 & 95.05 \\
\hline Totals & & 100.00 \\
\hline
\end{tabular}




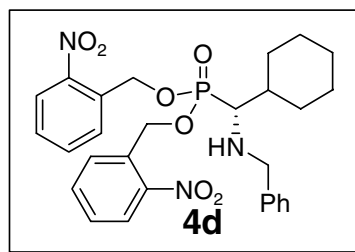

Chiral HPLC: Chiralcel OD, 95:05 Hex:EtOH, $1.0 \mathrm{ml} / \mathrm{min}, 208 \mathrm{~nm}$

racemic

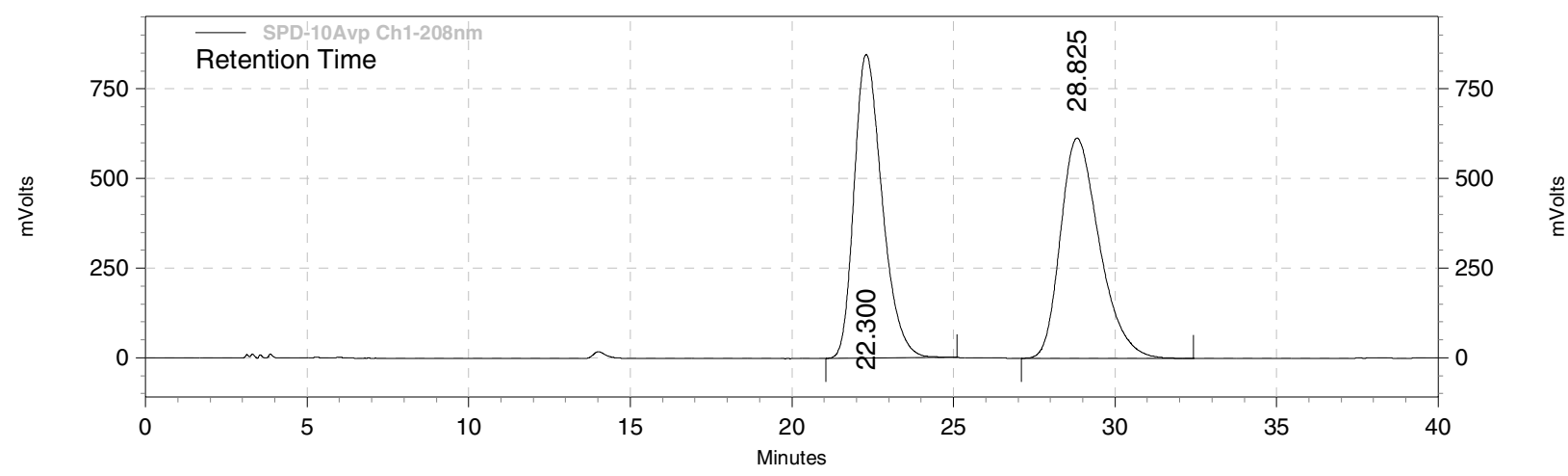

SPD-10Avp Ch1-208nm Results

\begin{tabular}{rrrr|} 
Retention Time & Area & Area Percent \\
\hline 22.30 & 51079939 & 49.83 \\
28.82 & 51423601 & 50.17 \\
\hline Totals & & 100.00 \\
\hline
\end{tabular}

$90 \% e e$

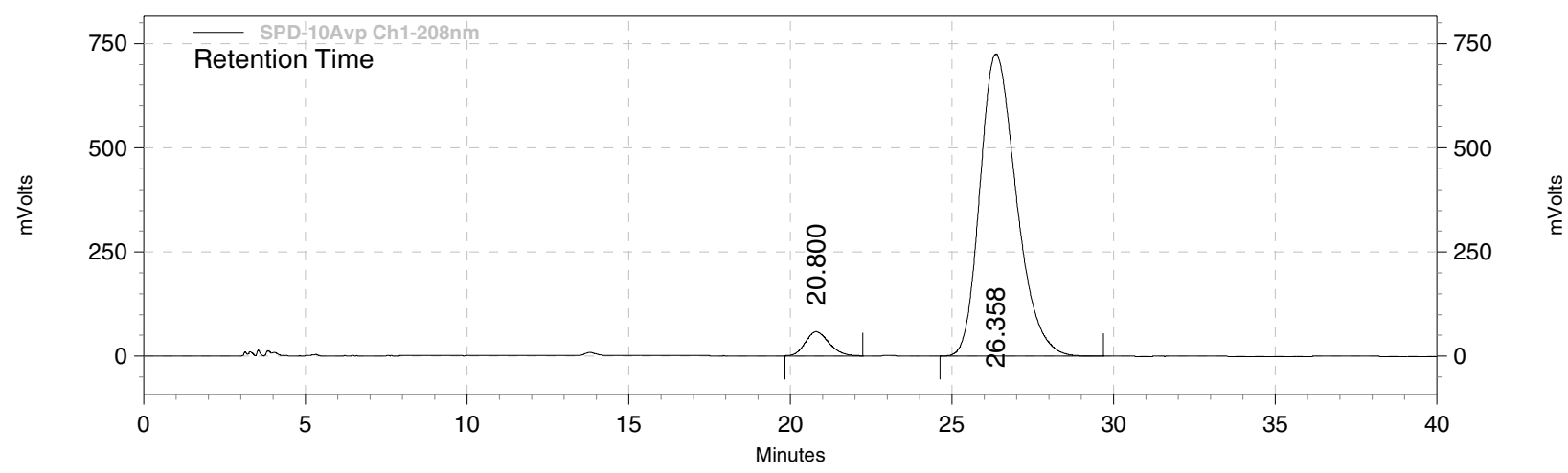

SPD-10Avp Ch1-208nm Results

\begin{tabular}{rrrr|} 
Retention Time & Area & Area Percent \\
\hline 20.80 & 2960389 & 4.97 \\
26.36 & 56664704 & 95.03 \\
\hline Totals & & 100.00 \\
\hline
\end{tabular}




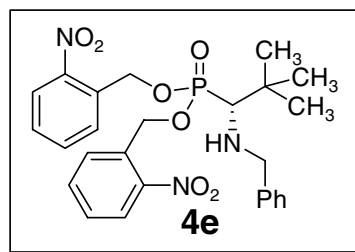

Chiral HPLC: Chiralcel OD, 95:05 Hex:EtOH, $1.0 \mathrm{ml} / \mathrm{min}, 208$ nm

racemic

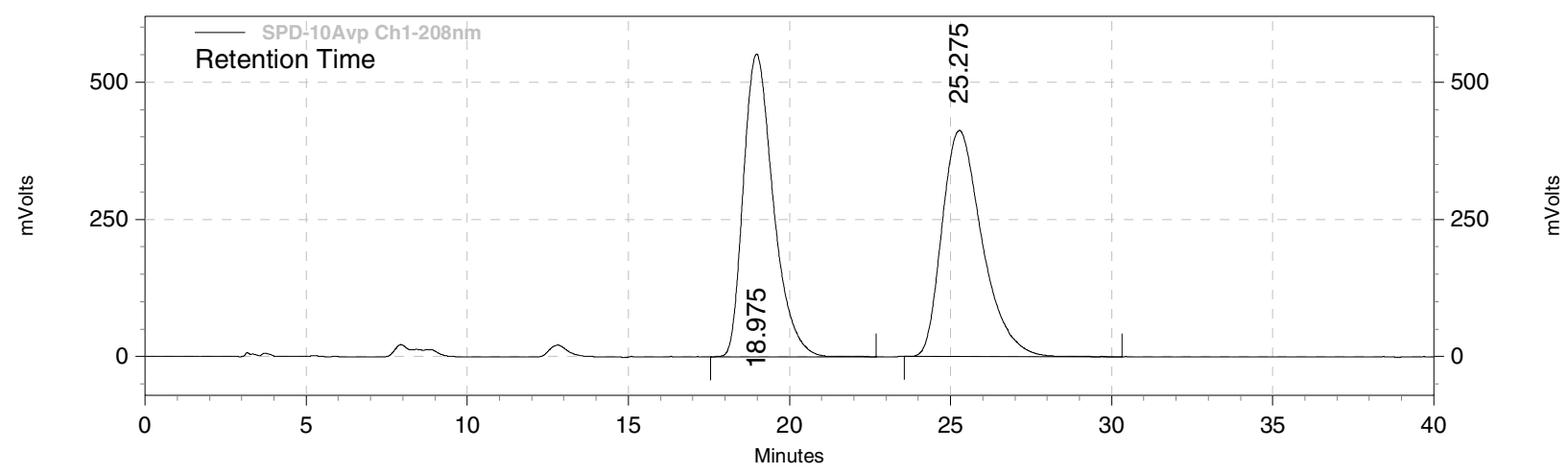

SPD-10Avp Ch1-208nm Results

Retention Time

Area

Area Percent

18.98
25.27

35576033

49.89

25.27

35730747

50.11

\begin{tabular}{|r|r|r|}
\hline Totals & 71306780 & 100.00 \\
\hline
\end{tabular}

$93 \% e e$

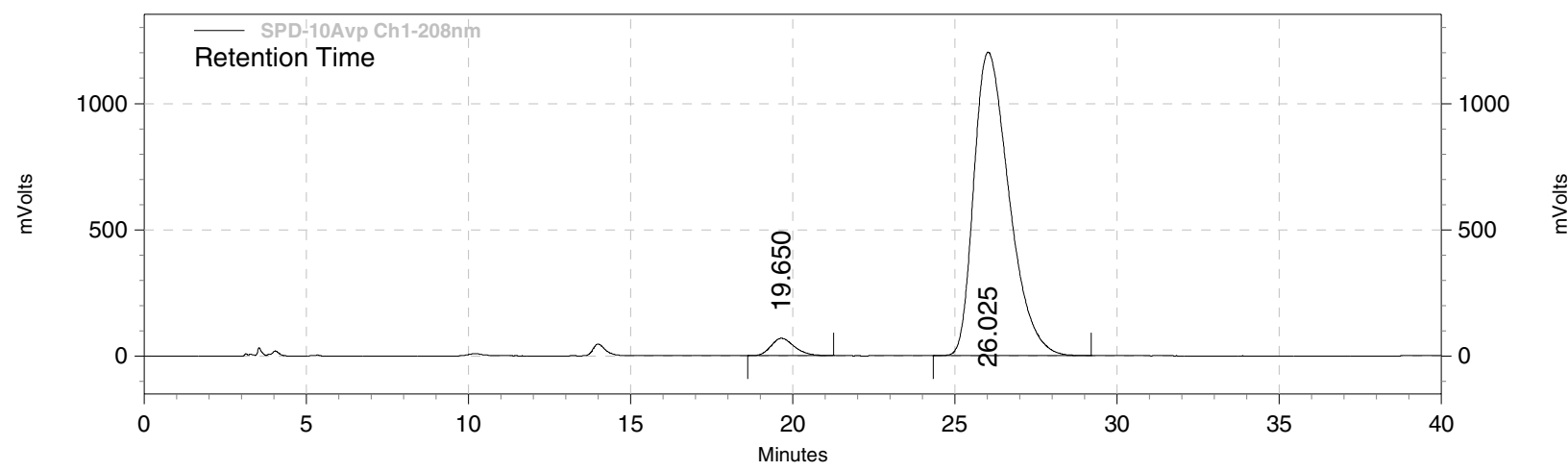

SPD-10Avp Ch1-208nm Results

\begin{tabular}{r|r|r|}
\multicolumn{1}{r}{ Retention Time } & Area & Area Percent \\
\hline 19.65 & 3511188 & 3.72 \\
26.02 & 90866874 & 96.28 \\
\hline Totals & 94378062 & 100.00 \\
\hline
\end{tabular}




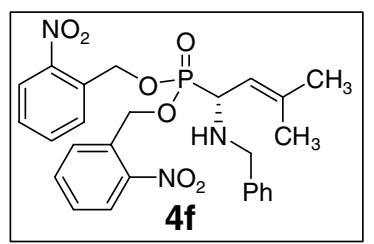

Chiral HPLC: Chiralcel OD, 97:03 Hex:EtOH, $1.0 \mathrm{ml} / \mathrm{min}, 208 \mathrm{~nm}$

racemic

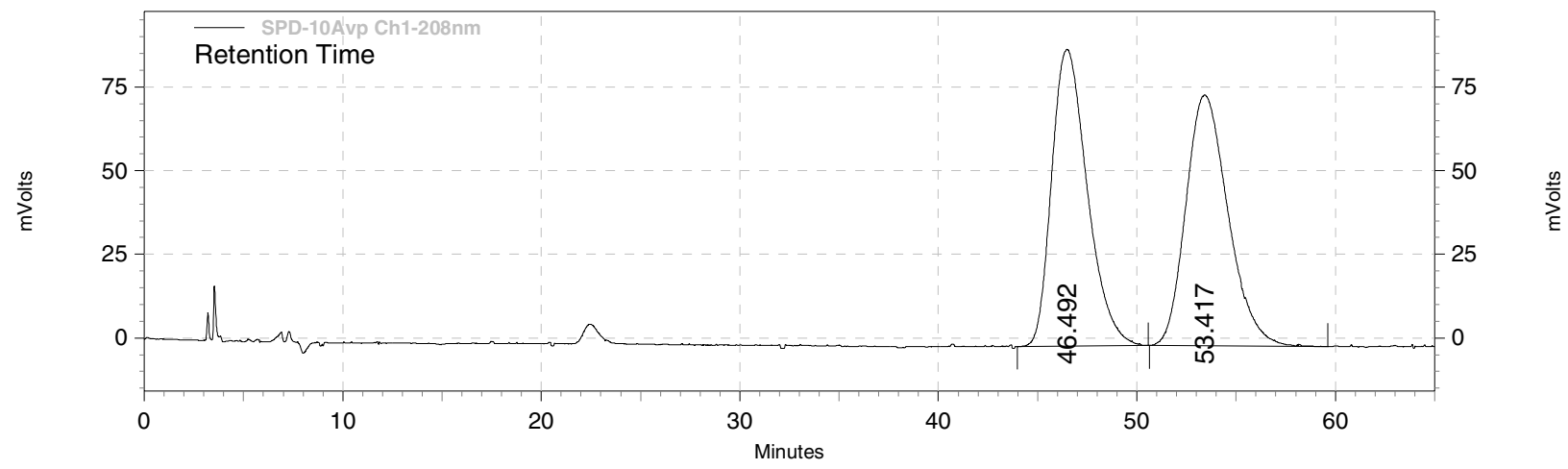

SPD-10Avp Ch1-208nm Results

Retention Time

46.49

Area

Area Percent

53.42

11054504

49.90

11098358

50.10

\begin{tabular}{|r|r|r|}
\hline Totals & 22152862 & 100.00 \\
\hline
\end{tabular}

$82 \% e e$

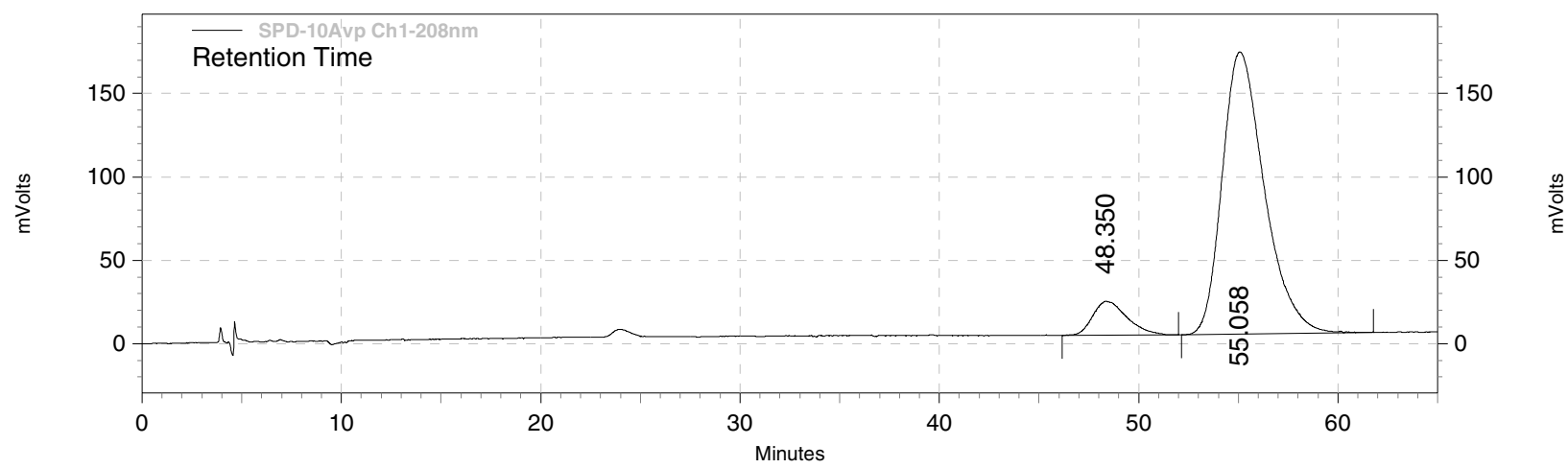

SPD-10Avp Ch1-208nm Results

Retention Time

Area

Area Percent

48.35

2431411

8.91

55.06

24851774

91.09

Totals 


\section{4f after recrystallization to $99 \% e e$}

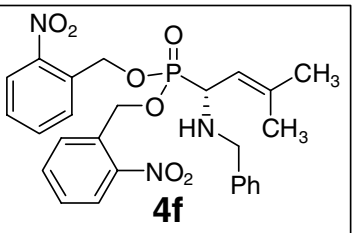

\section{Chiral HPLC: Chiralcel OD, 97:03 Hex:EtOH, $1.0 \mathrm{ml} / \mathrm{min}, 208$ nm}

racemic

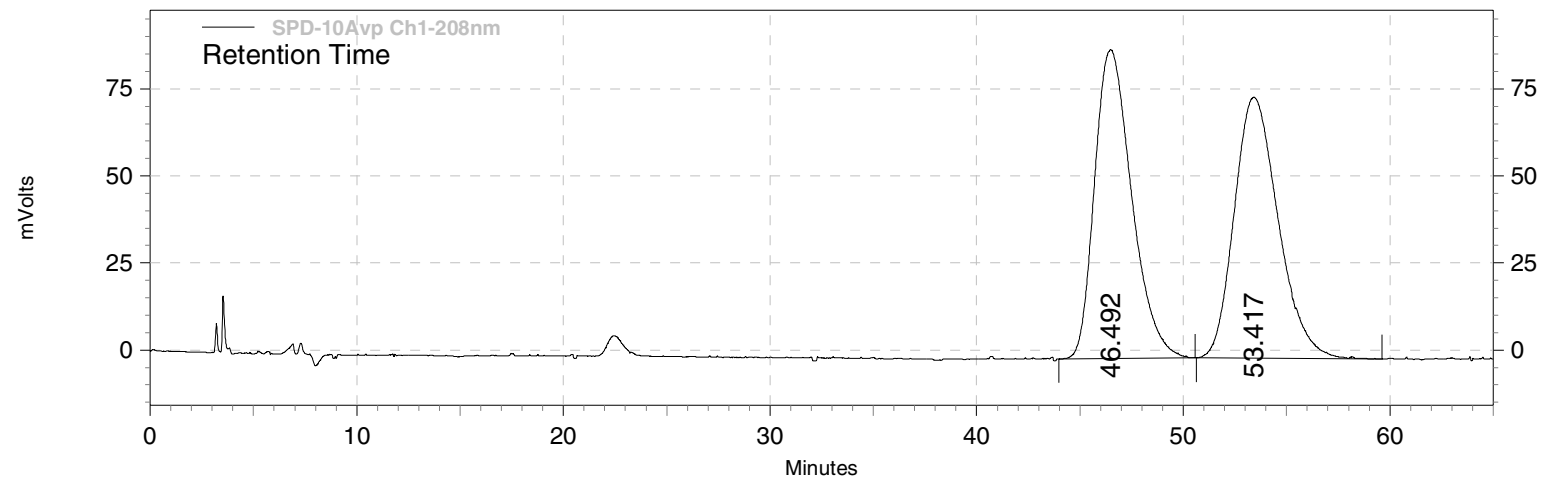

SPD-10Avp Ch1-208nm Results

Retention Time

Area

Area Percent

46.49

11054504

49.90

53.42

11098358

50.10

\begin{tabular}{|r|r|r|}
\hline Totals & 22152862 & 100.00 \\
\hline
\end{tabular}

$99 \% e e$

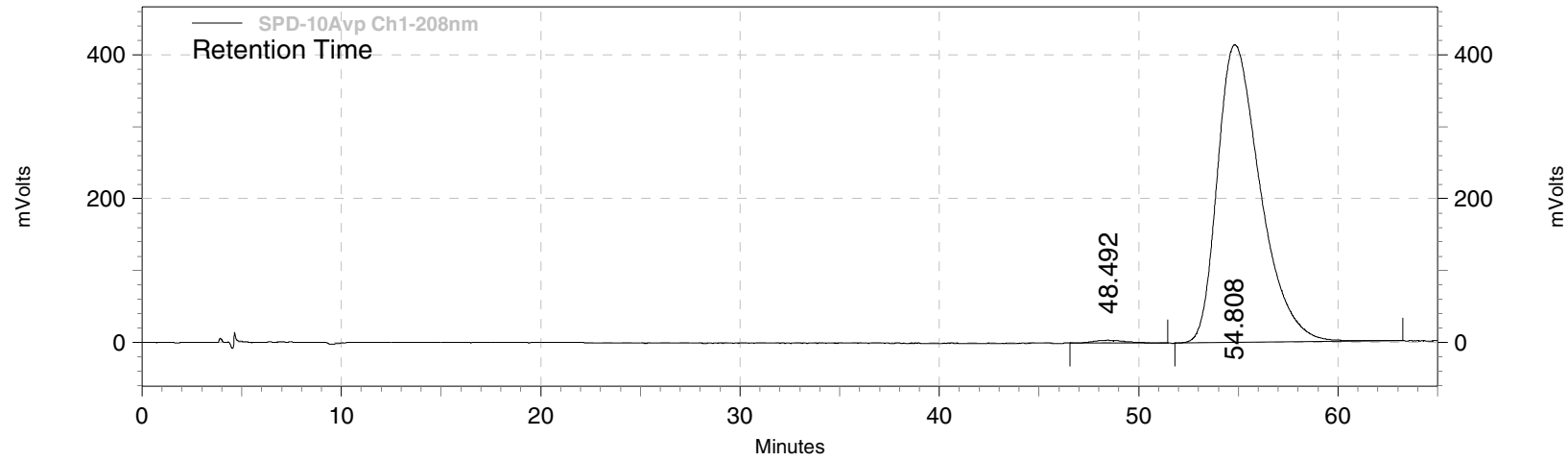

SPD-10Avp Ch1-208nm Results

Retention Time

Area

Area Percent

48.49

450591

0.72

54.81

62011750

99.28

\begin{tabular}{|r|r|r|}
\hline Totals & 62462341 & 100.00 \\
\hline
\end{tabular}




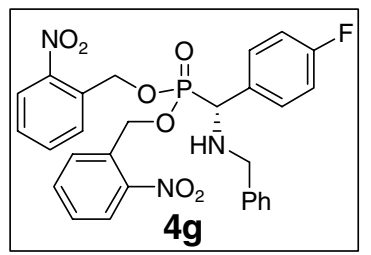

Chiral HPLC: Chiralpak AD, 70:30 Hex:IPA, 1.0 ml/min, 208 nm

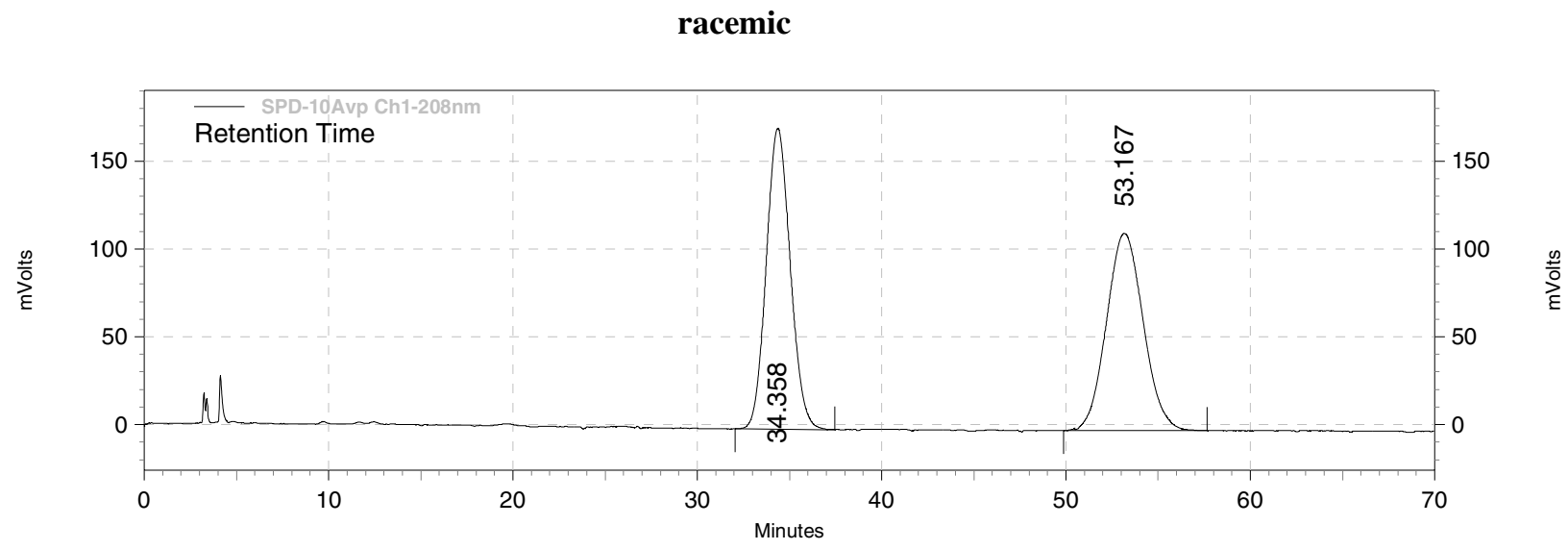

SPD-10Avp Ch1-208nm Results

Retention Time

Area

Area Percent

34.36

15546682

49.91

53.17

15604766

50.09

\begin{tabular}{|r|r|r|}
\hline Totals & 31151448 & 100.00 \\
\hline
\end{tabular}

$98 \% e e$

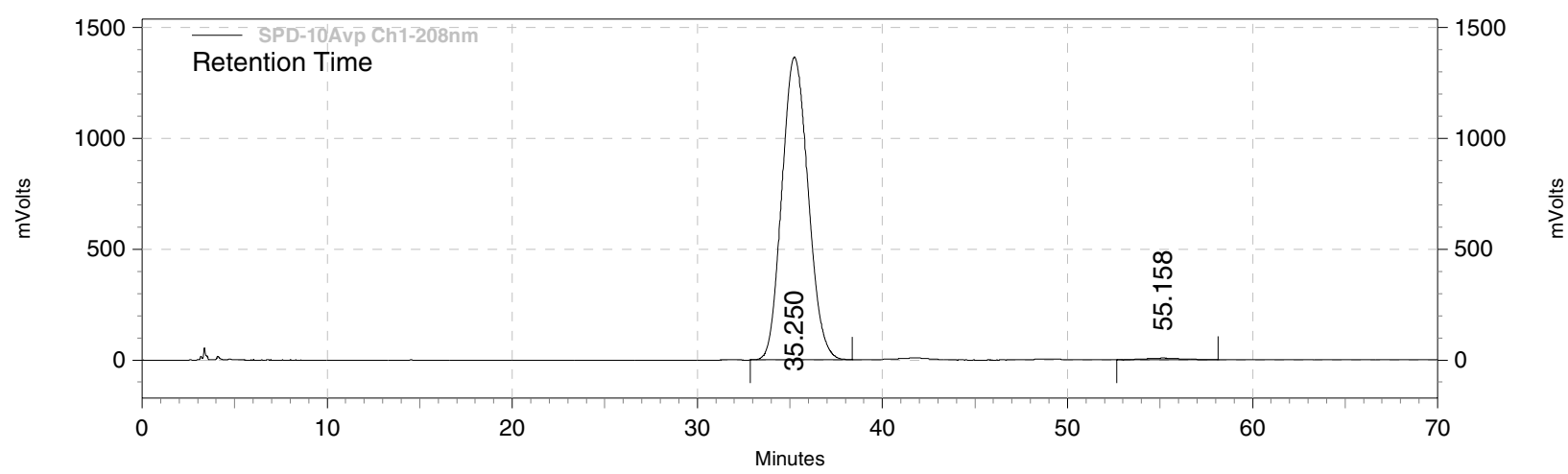

SPD-10Avp Ch1-208nm Results

\begin{tabular}{|c|c|c|}
\hline Retention Time & Area & Area Percent \\
\hline 35.25 & 134994947 & 99.25 \\
\hline 55.16 & 1017013 & 0.75 \\
\hline Totals & 136011960 & 100.00 \\
\hline
\end{tabular}




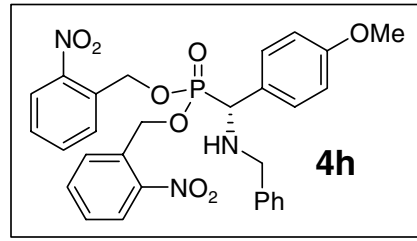

Chiral HPLC: Chiralpak AD, 70:30 Hex:IPA, $1.0 \mathrm{ml} / \mathrm{min}, 208 \mathrm{~nm}$

racemic

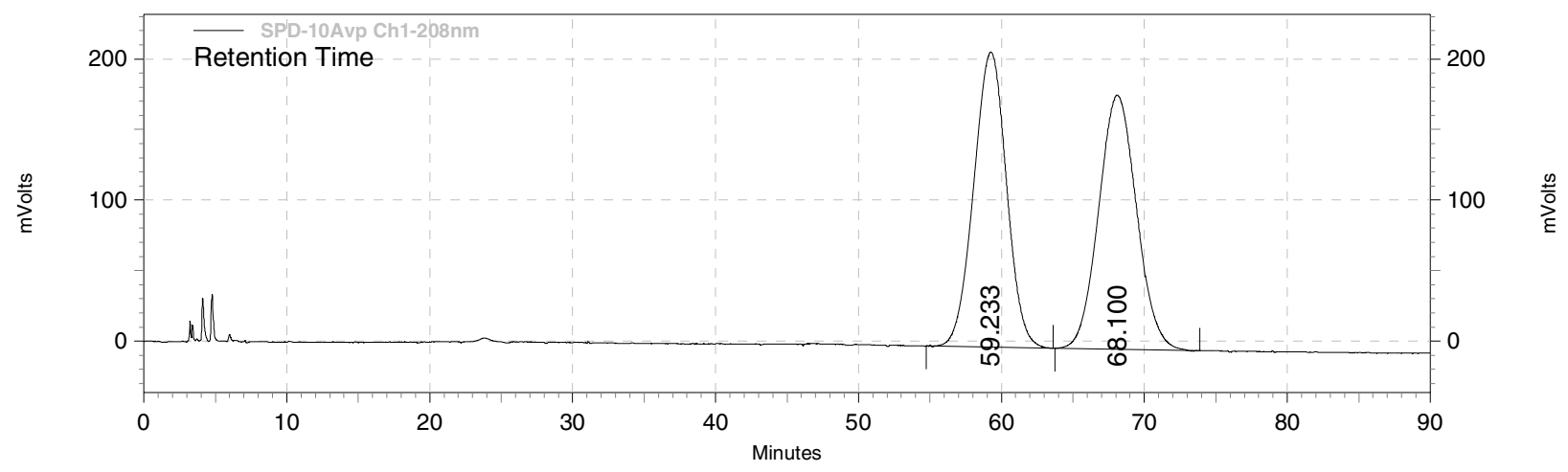

SPD-10Avp Ch1-208nm Results

Retention Time

59.23

Area

Area Percent

68.10

33425814

50.01

33412829

49.99

\begin{tabular}{|r|r|r|}
\hline Totals & 66838643 & 100.00 \\
\hline
\end{tabular}

$96 \% e e$

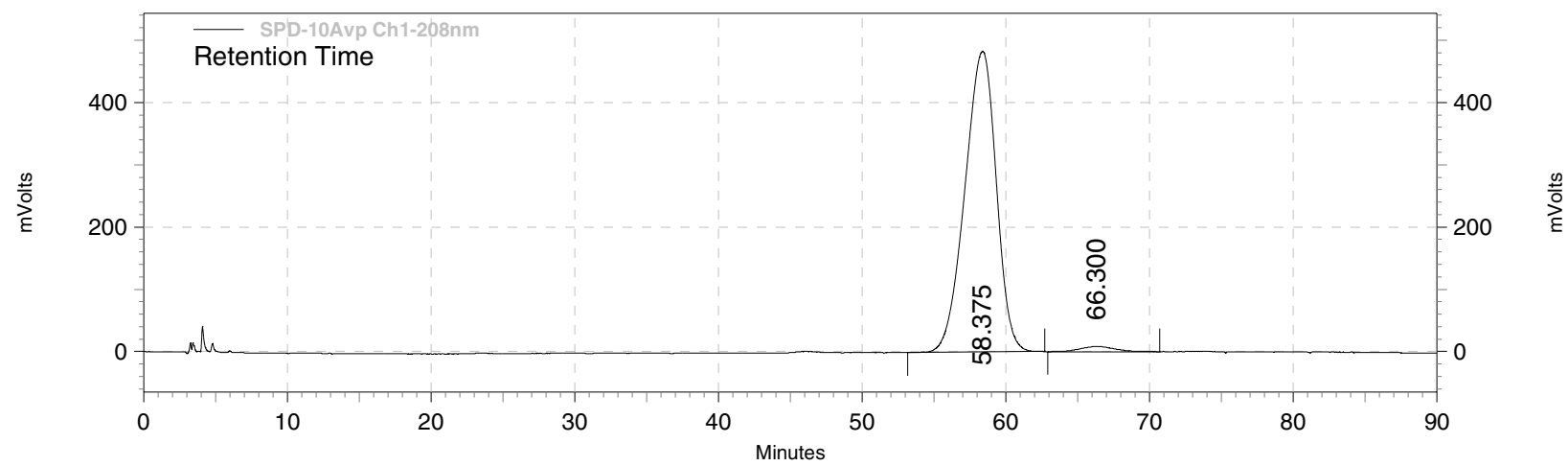

SPD-10Avp Ch1-208nm Results

Retention Time

58.38

Area

Area Percent

66.30

73305868

98.12

1405048

1.88

Totals 


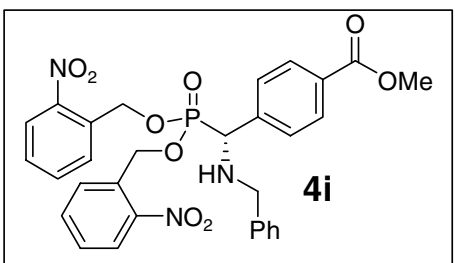

Chiral HPLC: Chiralpak AD, 70:30 Hex:IPA, $1.5 \mathrm{ml} / \mathrm{min}, 208 \mathrm{~nm}$

racemic

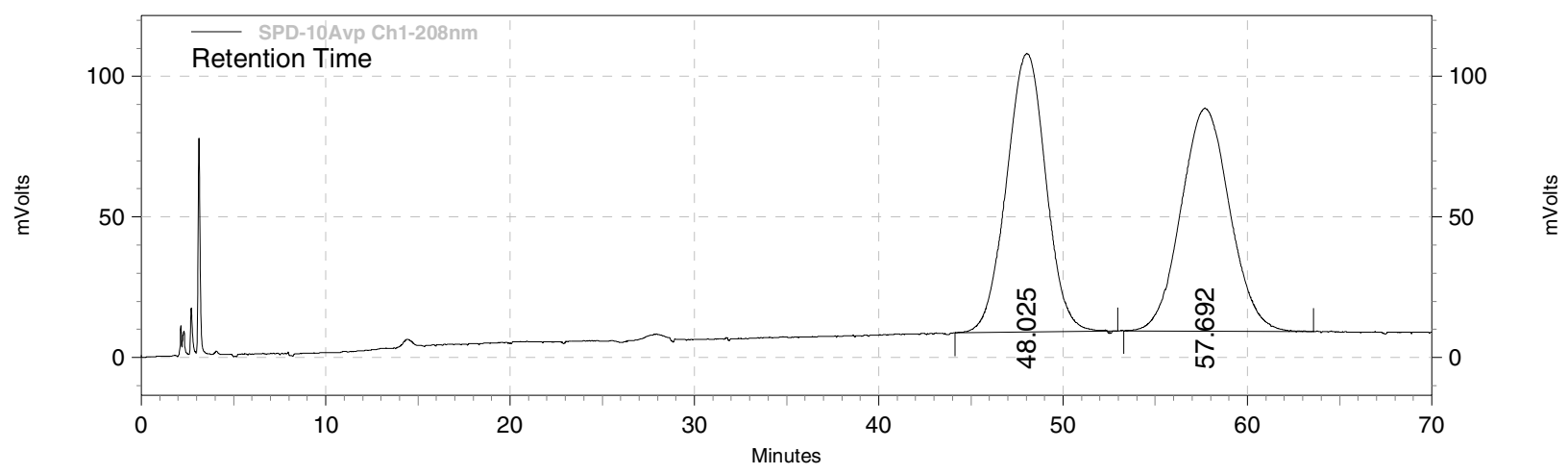

SPD-10Avp Ch1-208nm Results

Retention Time

Area

Area Percent

48.02 14709984

50.30

57.69 14536162

49.70

\begin{tabular}{|r|r|r|}
\hline Totals & 29246146 & 100.00 \\
\hline
\end{tabular}

$96 \% e e$

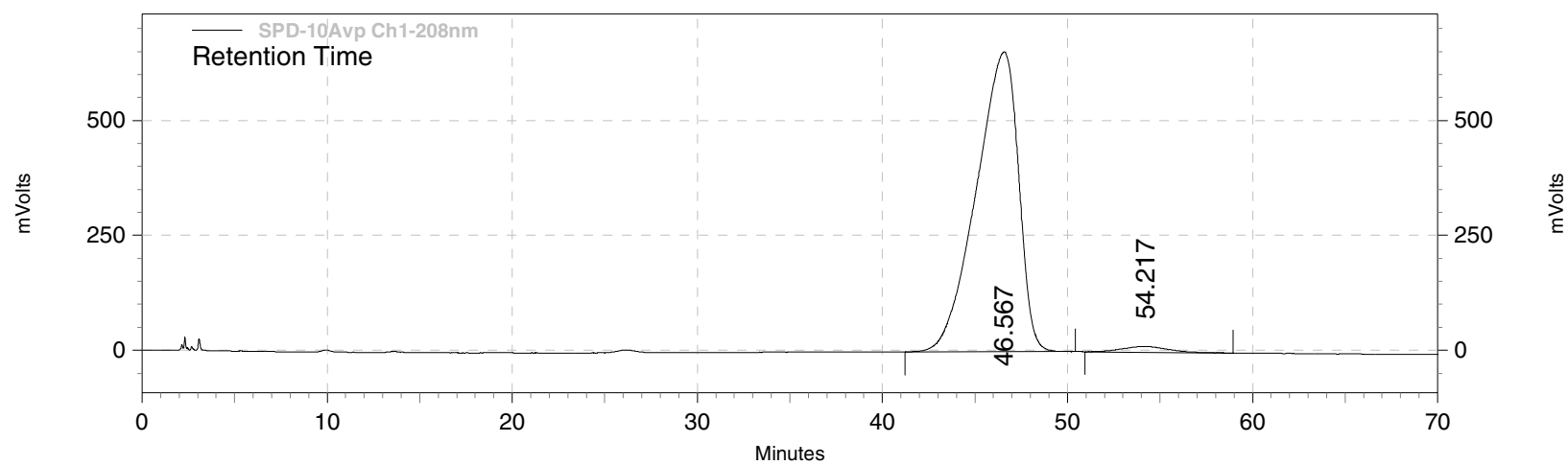

SPD-10Avp Ch1-208nm Results

Retention Time

Area

Area Percent

46.57

103679118

54.22

2043643

1.93

\section{Totals}




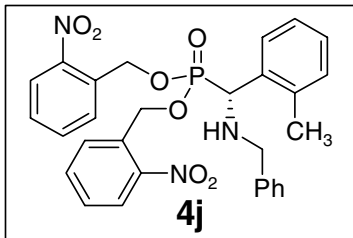

Chiral HPLC: Pirkle Covalent L-Leucine, 99:01 Hex:EtOH, $1.0 \mathrm{ml} / \mathrm{min}, 208$ nm

racemic

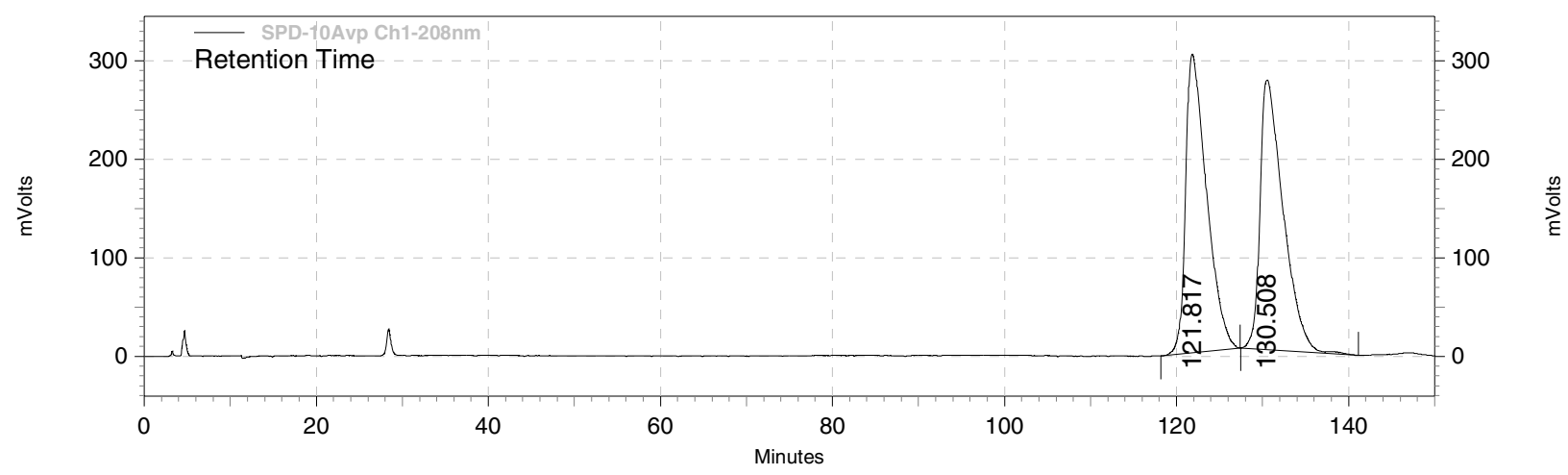

SPD-10Avp Ch1-208nm Results

Retention Time

\begin{tabular}{rrrr|}
\multicolumn{1}{r}{ Retention Time } & Area & Area Percent \\
\hline 121.82 & 51373316 & 50.15 \\
130.51 & 51070149 & 49.85 \\
& & \\
\hline Totals & 102443465 & 100.00 \\
\hline
\end{tabular}

Area Percent

$92 \% e e$

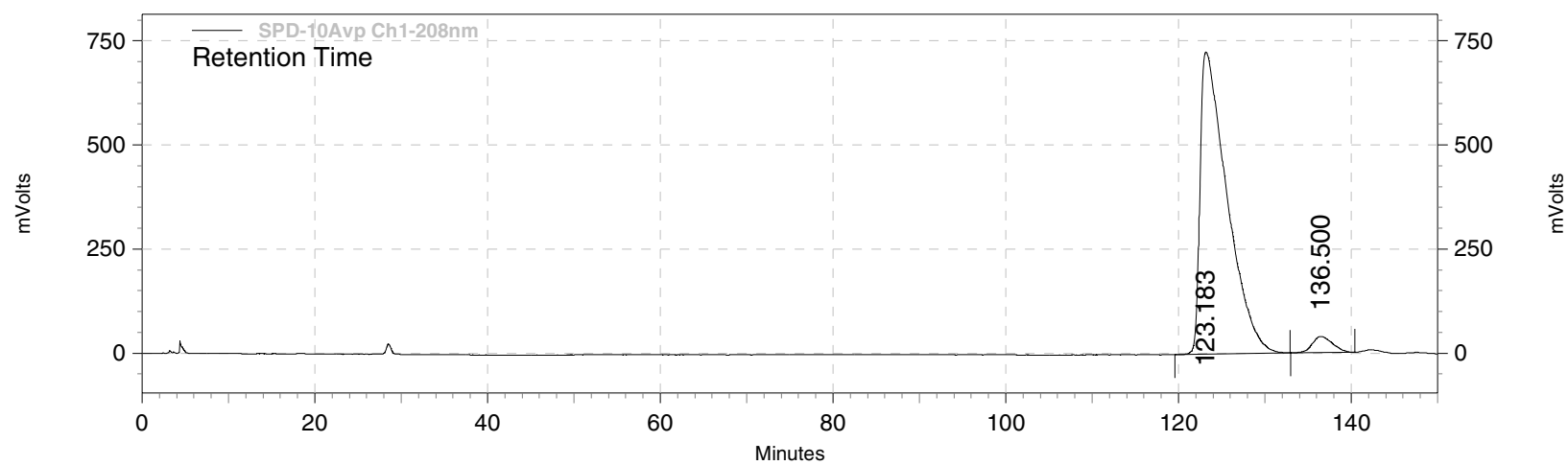

SPD-10Avp Ch1-208nm Results

\begin{tabular}{rrrr|}
\multicolumn{1}{r}{ Retention Time } & Area & Area Percent \\
\hline 123.18 & 157049533 & 96.12 \\
136.50 & 6336163 & 3.88 \\
\hline Totals & & 100.00 \\
\hline
\end{tabular}




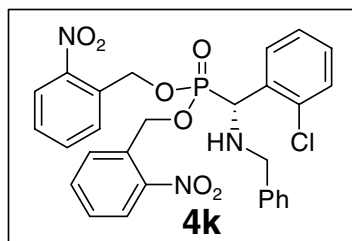

Chiral HPLC: Chiralpak AD, 70:30 Hex:IPA, 1.0 ml/min, 208 nm

racemic

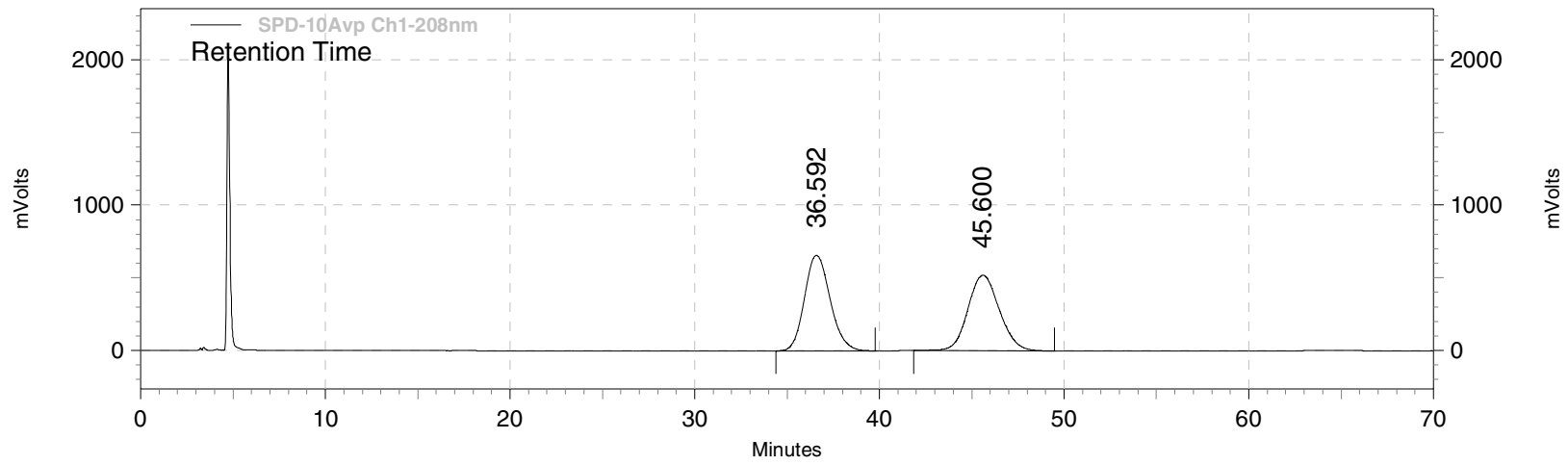

SPD-10Avp Ch1-208nm Results

Retention Time

36.59
45.60

Area

Area Percent

45.60

63501810

50.73

61674936

49.27

\begin{tabular}{|r|r|r|}
\hline Totals & 125176746 & 100.00 \\
\hline
\end{tabular}

$96 \% e e$

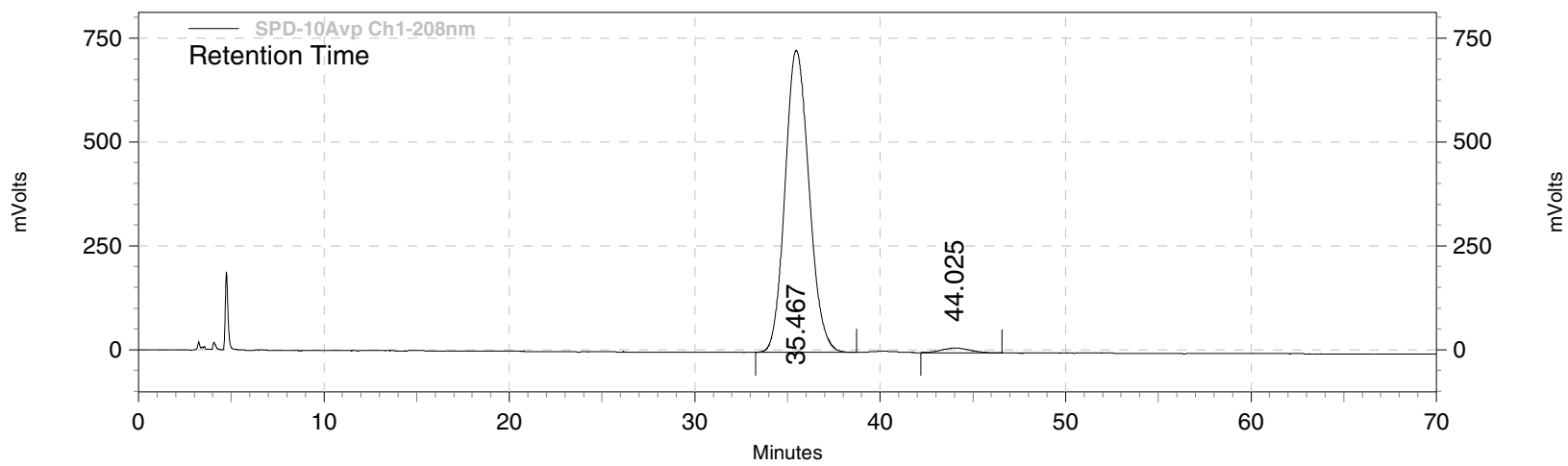

SPD-10Avp Ch1-208nm Results

\begin{tabular}{rrrr|}
\multicolumn{1}{r}{ Retention Time } & Area & Area Percent \\
\hline 35.47 & 65635348 & 98.26 \\
44.02 & 1161964 & 1.74 \\
\hline Totals & & 100.00 \\
\hline
\end{tabular}




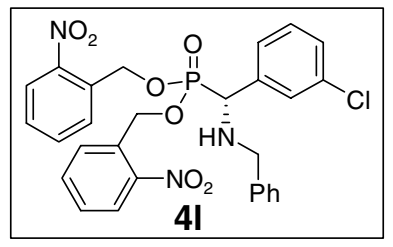

Chiral HPLC: Chiralpak AD, 87:13 Hex:EtOH, 1.0 ml/min, 208 nm

racemic

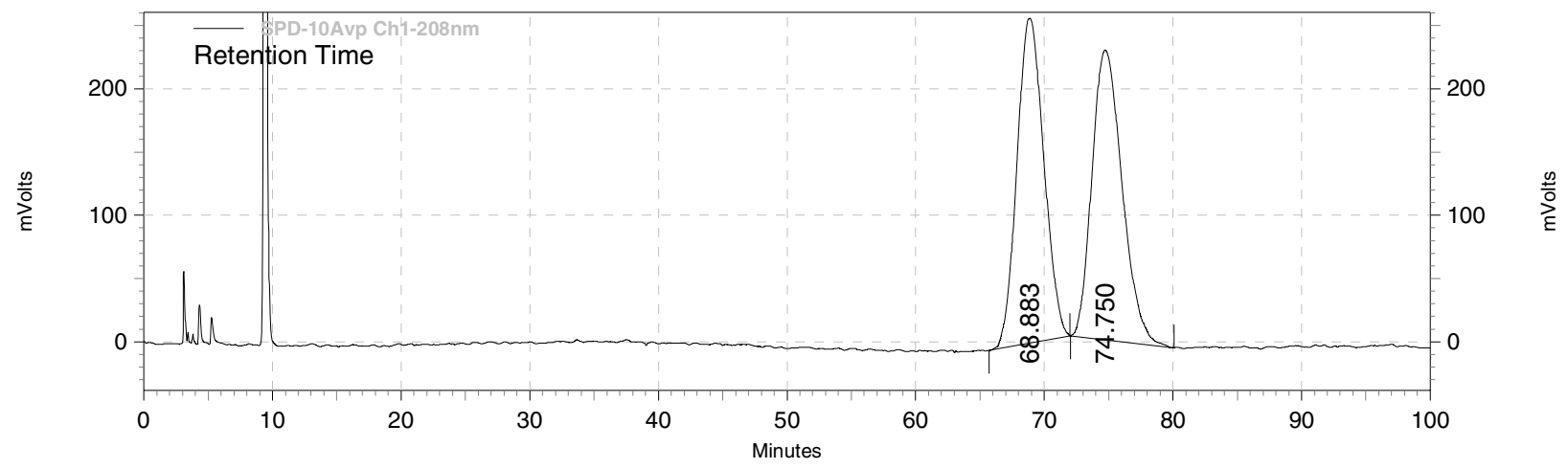

SPD-10Avp Ch1-208nm Results

Retention Time

68.88

Area

Area Percent

74.75

37288415

50.07

Totals

37389808

50.07

\begin{tabular}{|r|r|r|}
\hline Totals & 74678223 & 100.00 \\
\hline
\end{tabular}

$98 \% e e$

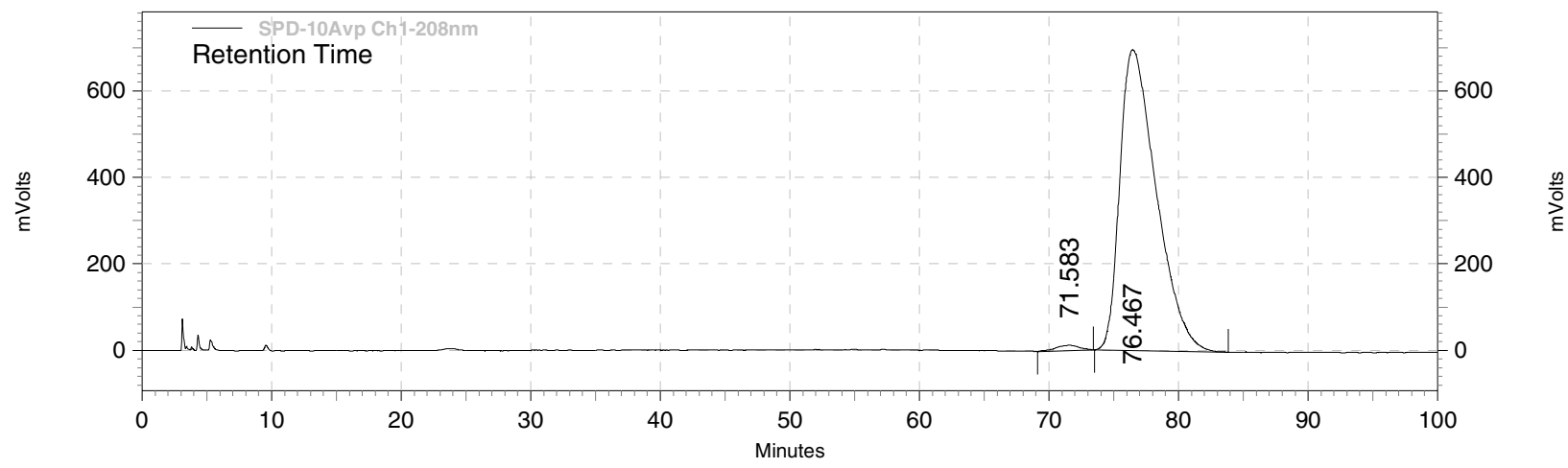

SPD-10Avp Ch1-208nm Results

Retention Time

Area

Area Percent

71.58

1518577

76.47

135699963

1.11

98.89

Totals

137218540

100.00 


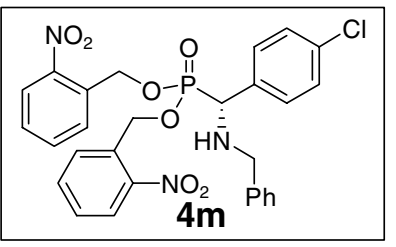

Chiral HPLC: Chiralpak AD, 70:30 Hex:IPA, $1.0 \mathrm{ml} / \mathrm{min}, 208 \mathrm{~nm}$

racemic

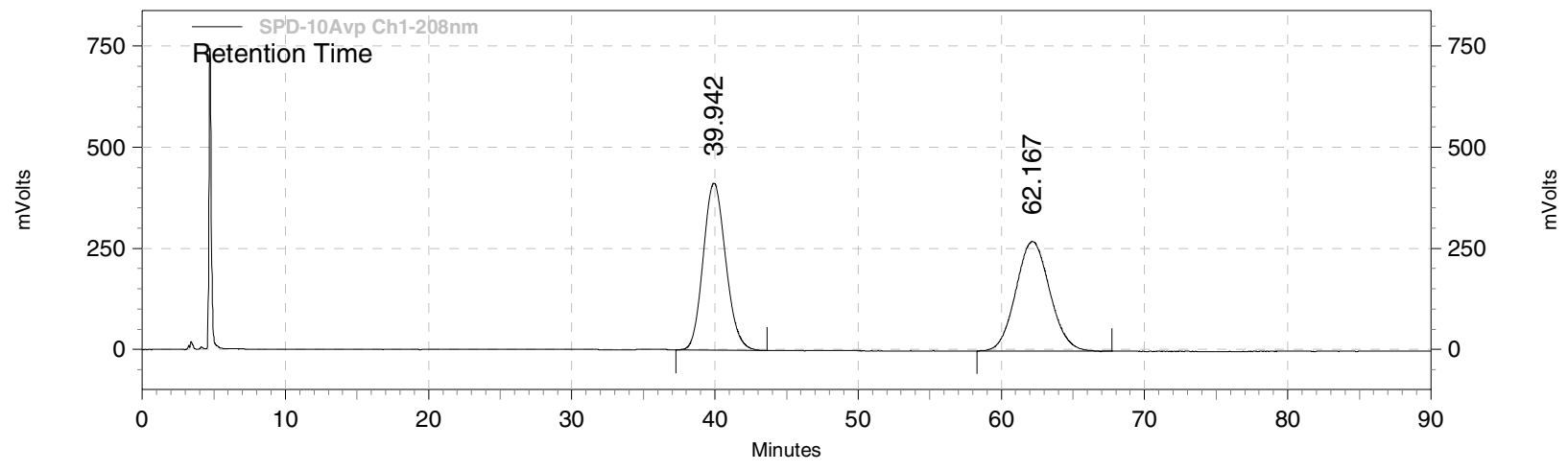

SPD-10Avp Ch1-208nm Results

Retention Time

39.94

Area

Area Percent

62.17

44937310

50.18

44613811

49.82

\begin{tabular}{|r|r|r|}
\hline Totals & 89551121 & 100.00 \\
\hline
\end{tabular}

$99 \% e e$

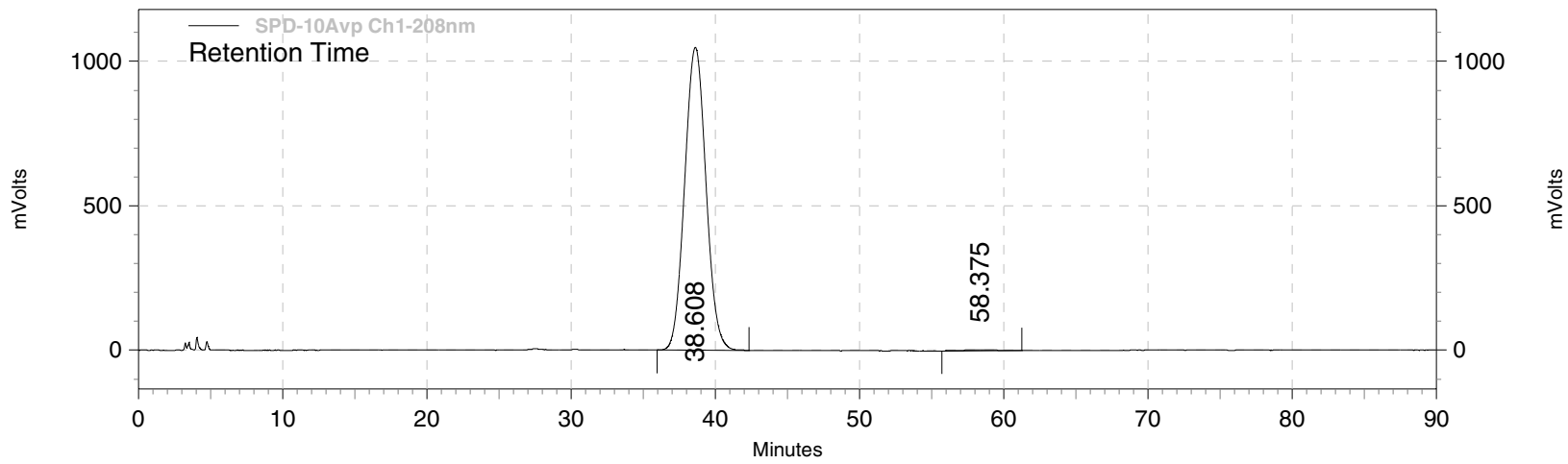

SPD-10Avp Ch1-208nm Results

Retention Time

Area

Area Percent

38.61

107654959

520790

99.52

58.38

08175749

0.48

Totals 


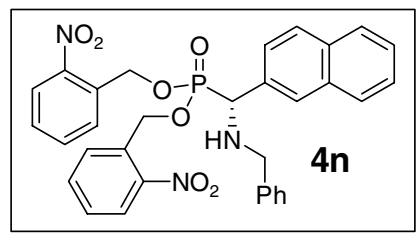

Chiral HPLC: Chiralpak AD, 70:30 Hex:IPA, 1.0 ml/min, 208 nm

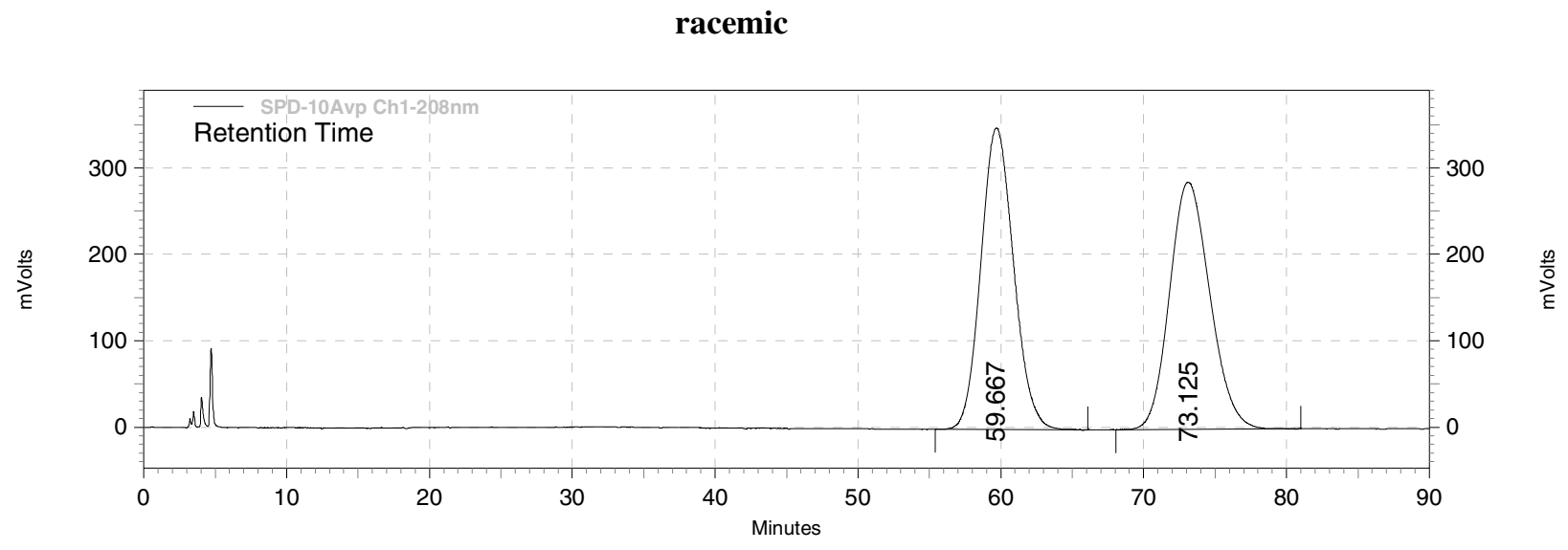

SPD-10Avp Ch1-208nm Results

\begin{tabular}{r|r|r|}
$r$ & Area & Area Percent \\
\hline Retention Time & 55845540 & 49.98 \\
59.67 & 55882584 & 50.02 \\
\hline Th.13 & & 100.00 \\
\hline
\end{tabular}

$98 \% e e$

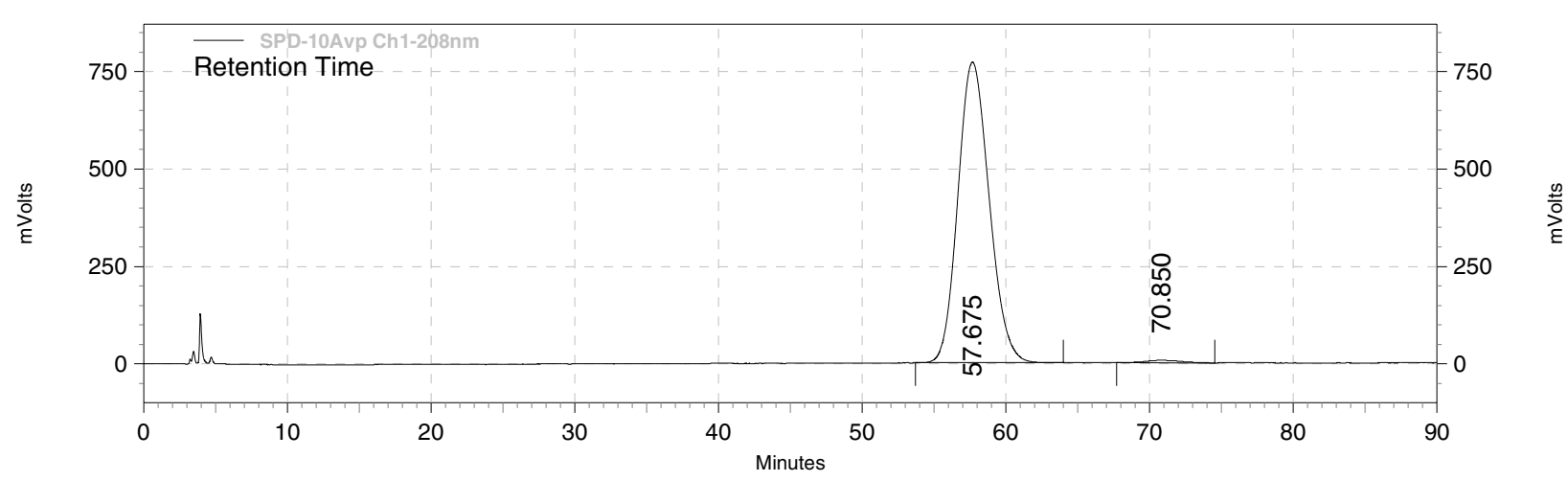

SPD-10Avp Ch1-208nm Results

Retention Time

Area

Area Percent

57.67

117044577

99.00

70.85

1177568

1.00

\section{Totals}




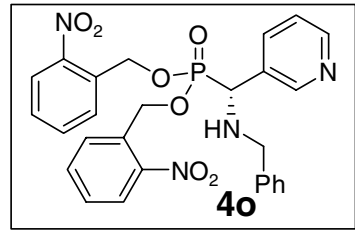

\section{Chiral HPLC: Chiralpak AS, 85:15 Hex:EtOH, $1.5 \mathrm{ml} / \mathrm{min}, 208$ nm}

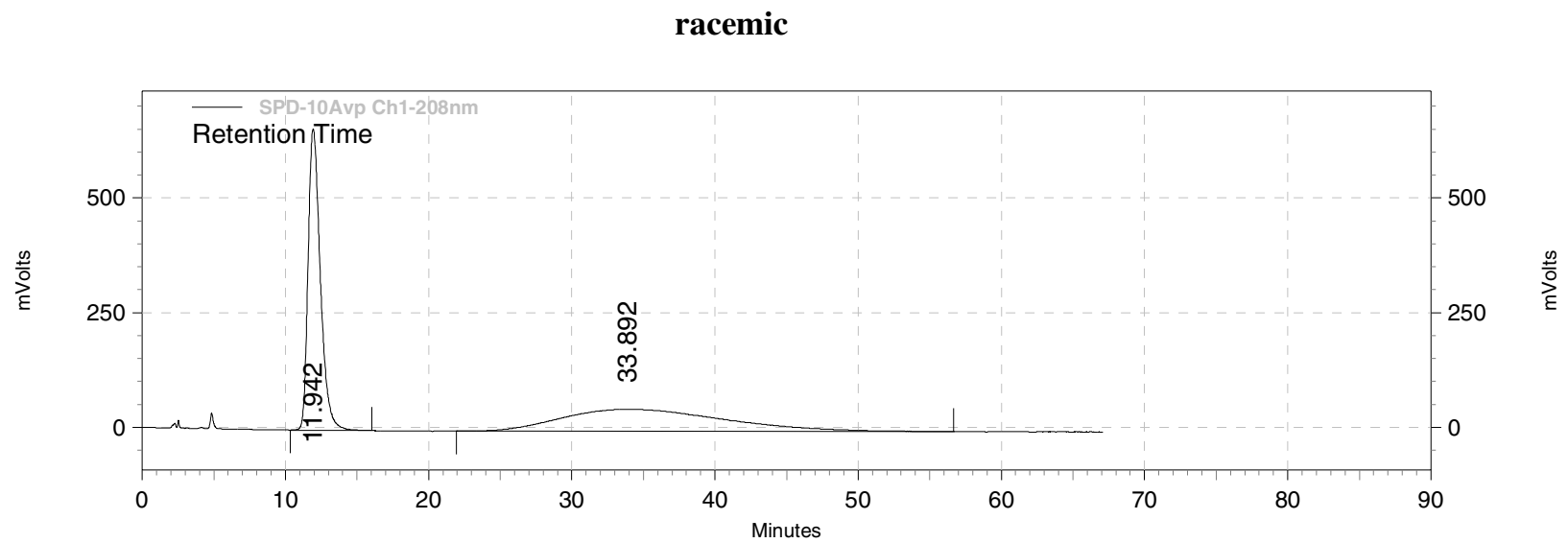

SPD-10Avp Ch1-208nm Results

\begin{tabular}{rrrr|} 
Retention Time & Area & Area Percent \\
\hline 11.94 & 39008712 & 50.66 \\
33.89 & 37998451 & 49.34 \\
\hline Totals & 77007163 & 100.00 \\
\hline
\end{tabular}

$96 \% e e$

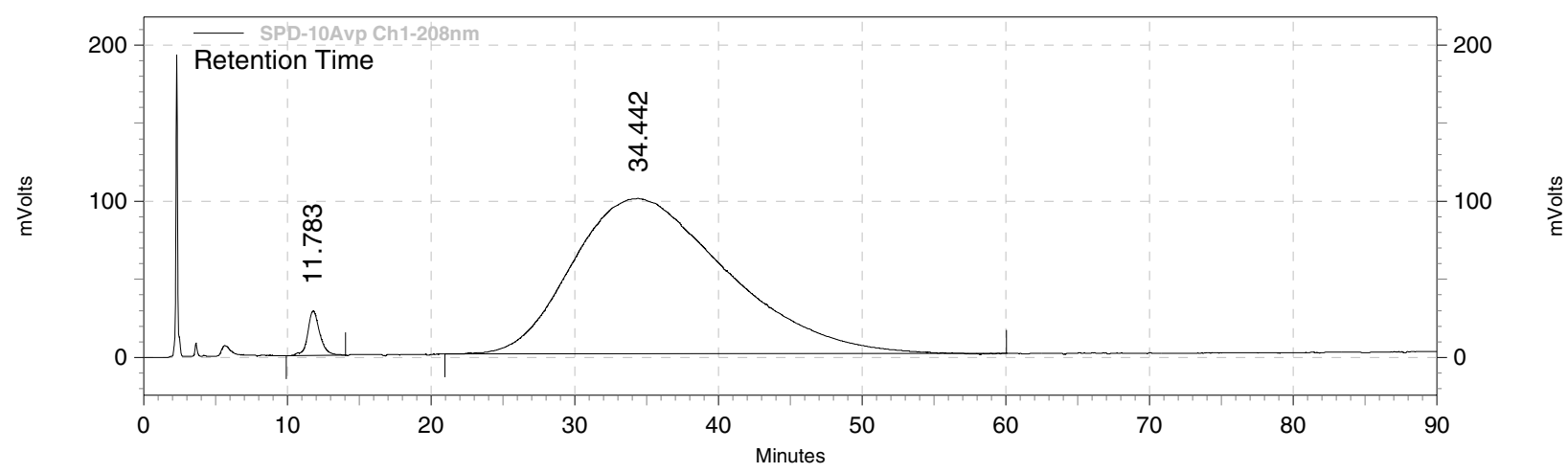

SPD-10Avp Ch1-208nm Results

Retention Time Area Area Percent

$\begin{array}{rrr}11.78 & 1693523 & 2.22 \\ 34.44 & 74471263 & 97.78\end{array}$

Totals 


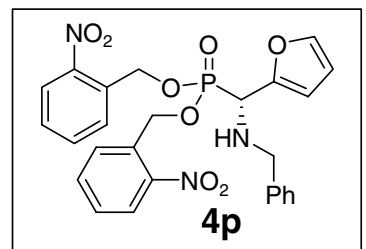

Chiral HPLC: Chiralpak AD, 70:30 Hex:IPA, $1.0 \mathrm{ml} / \mathrm{min}, 208 \mathrm{~nm}$

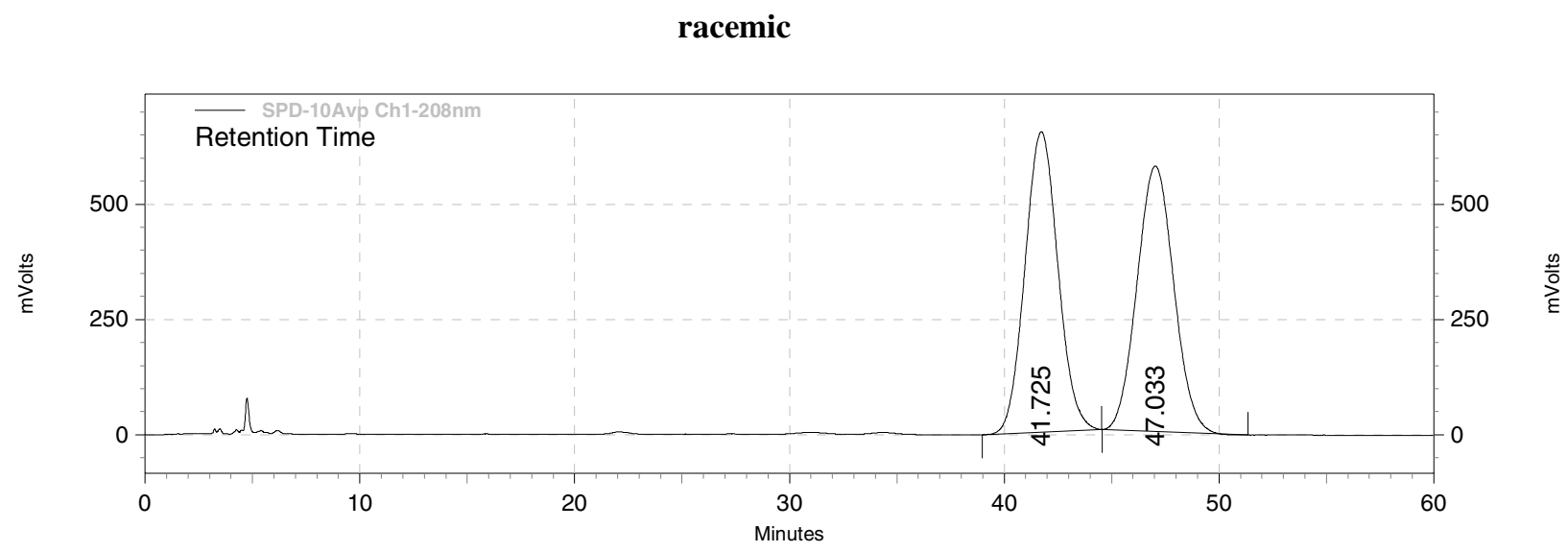

SPD-10Avp Ch1-208nm Results

Retention Time

41.73

Area

Area Percent

47.03

69586958

50.35

68607562

49.65

\begin{tabular}{|r|r|r|}
\hline Totals & 138194520 & 100.00 \\
\hline
\end{tabular}

$92 \% e e$

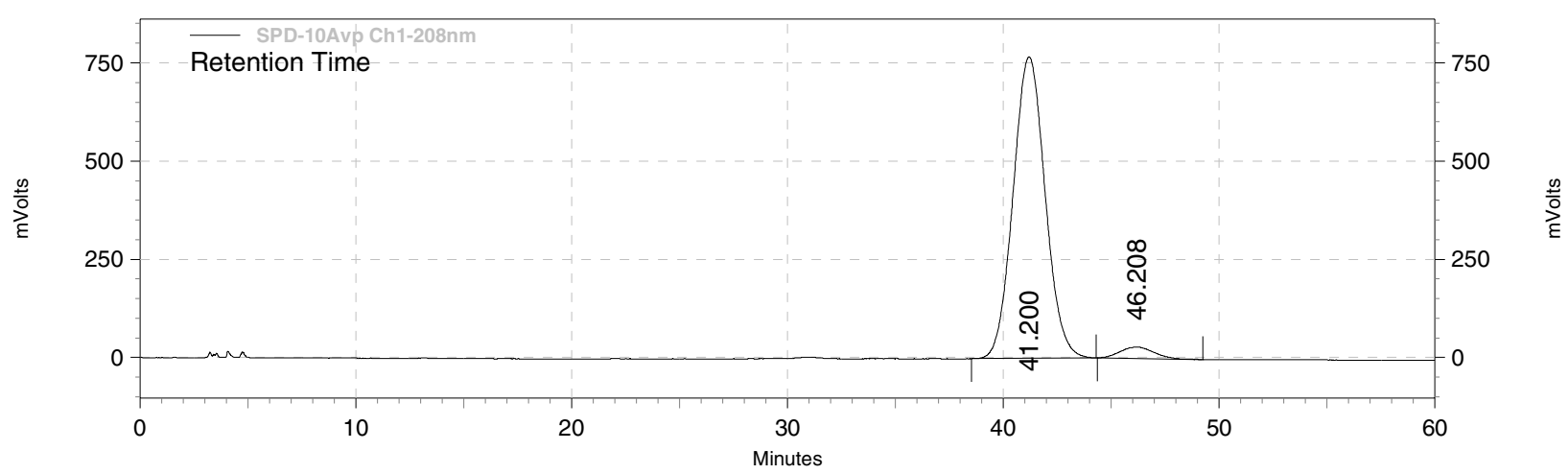

SPD-10Avp Ch1-208nm Results

\begin{tabular}{|c|c|c|}
\hline Retention Time & Area & Area Percent \\
\hline 41.20 & 79176926 & 96.15 \\
\hline 46.21 & 3168525 & 3.85 \\
\hline Totals & 82345451 & 100.00 \\
\hline
\end{tabular}




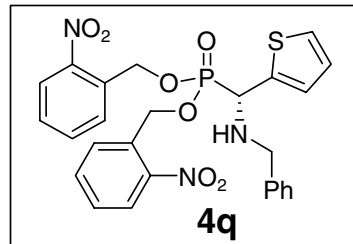

Chiral HPLC: Chiralcel OD, 95:05 Hex:EtOH, 1.0 ml/min, $208 \mathrm{~nm}$

racemic

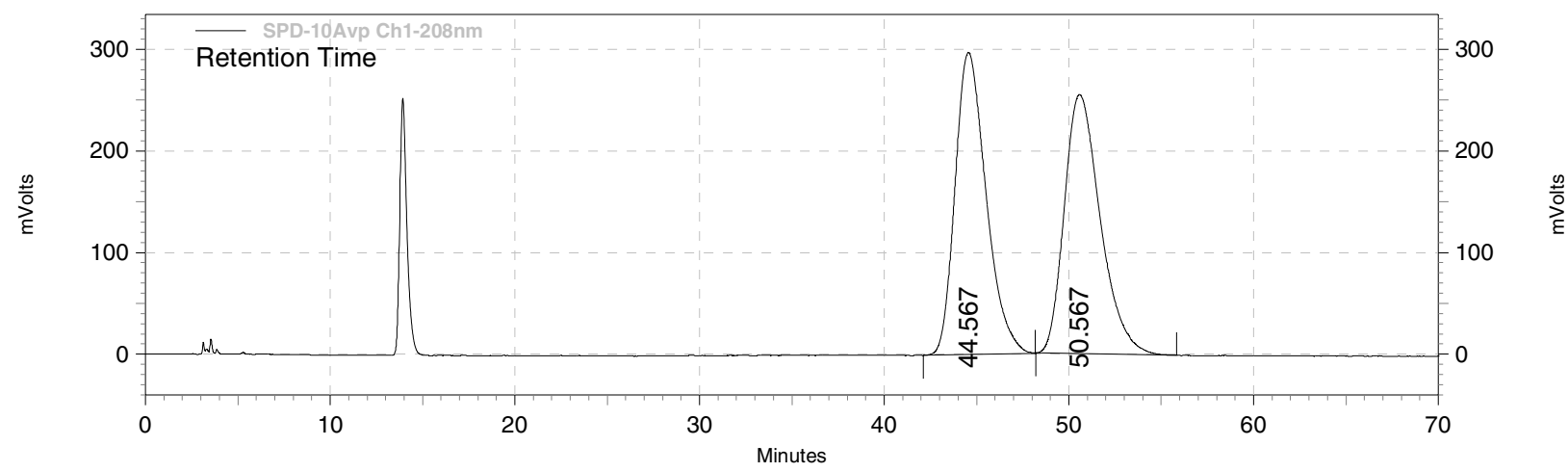

SPD-10Avp Ch1-208nm Results

Retention Time

44.57

Area

Area Percent

50.57

34695095

50.06

34617888

49.94

\begin{tabular}{|r|r|r|}
\hline Totals & 69312983 & 100.00 \\
\hline
\end{tabular}

$94 \% e e$

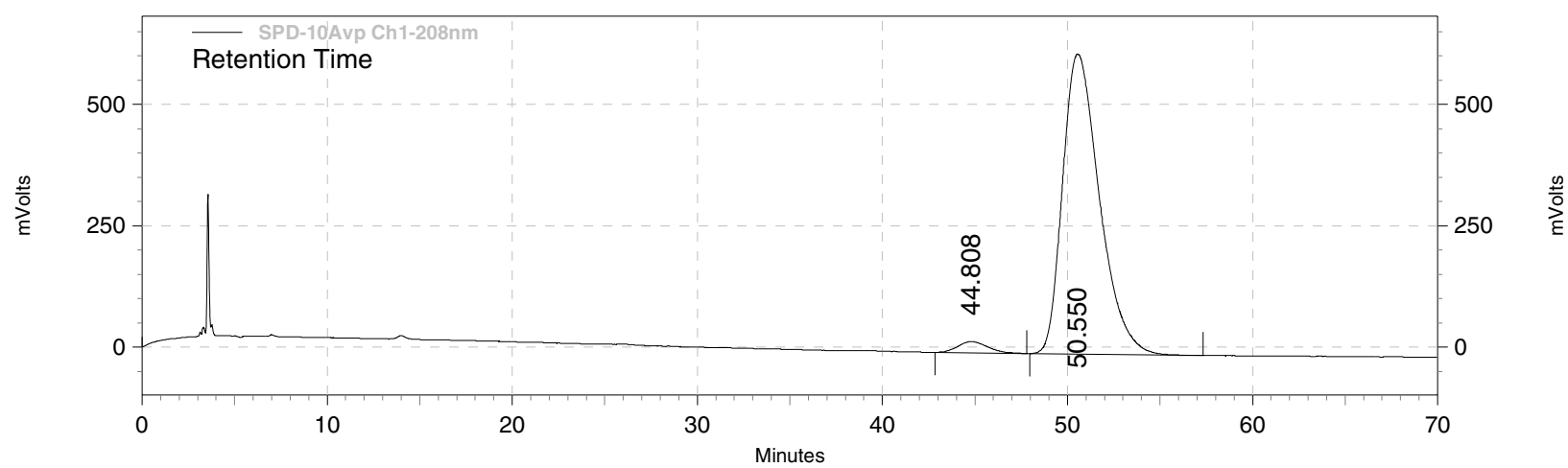

SPD-10Avp Ch1-208nm Results

\begin{tabular}{rrrr|}
\multicolumn{1}{r}{ Retention Time } & Area & Area Percent \\
\hline 44.81 & 2542692 & 2.89 \\
50.55 & 85473129 & 97.11 \\
\hline Totals & & 100.00 \\
\hline
\end{tabular}




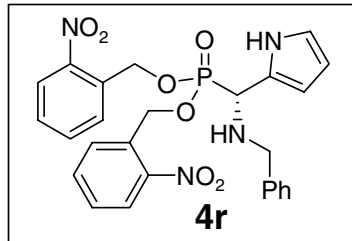

Chiral HPLC: Chiralpak AD, 70:30 Hex:IPA, 1.0 ml/min, 208 nm

racemic

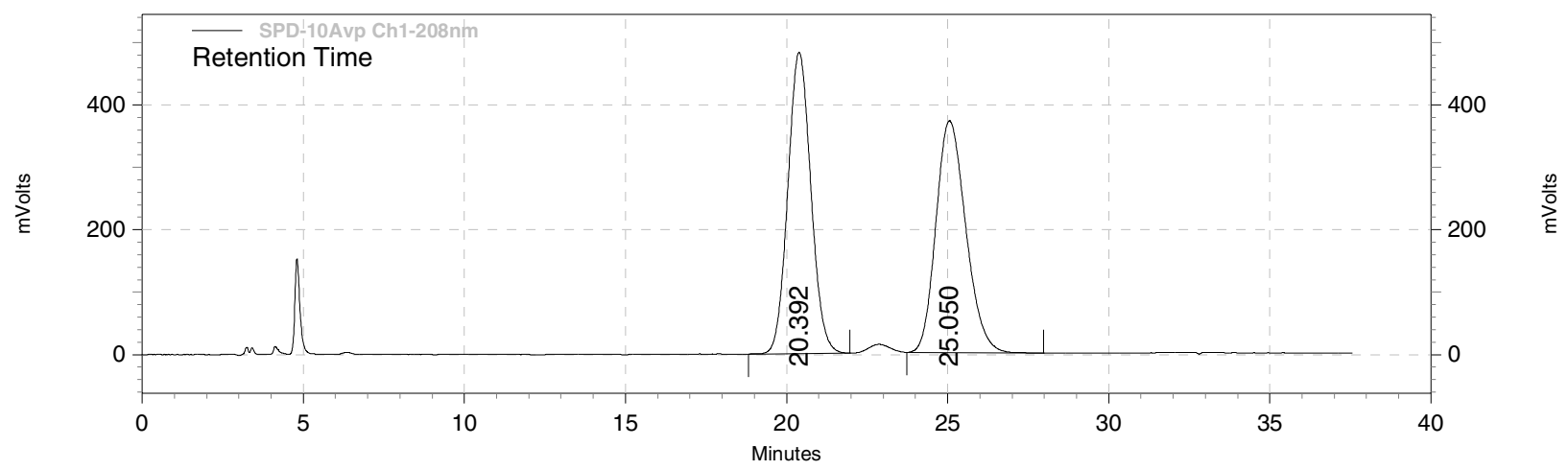

SPD-10Avp Ch1-208nm Results

Retention Time

20.39
25.05

Area

Area Percent

50.11

23919299

49.89

\begin{tabular}{|r|r|r|}
\hline Totals & 47941870 & 100.00 \\
\hline
\end{tabular}

$81 \% e e$

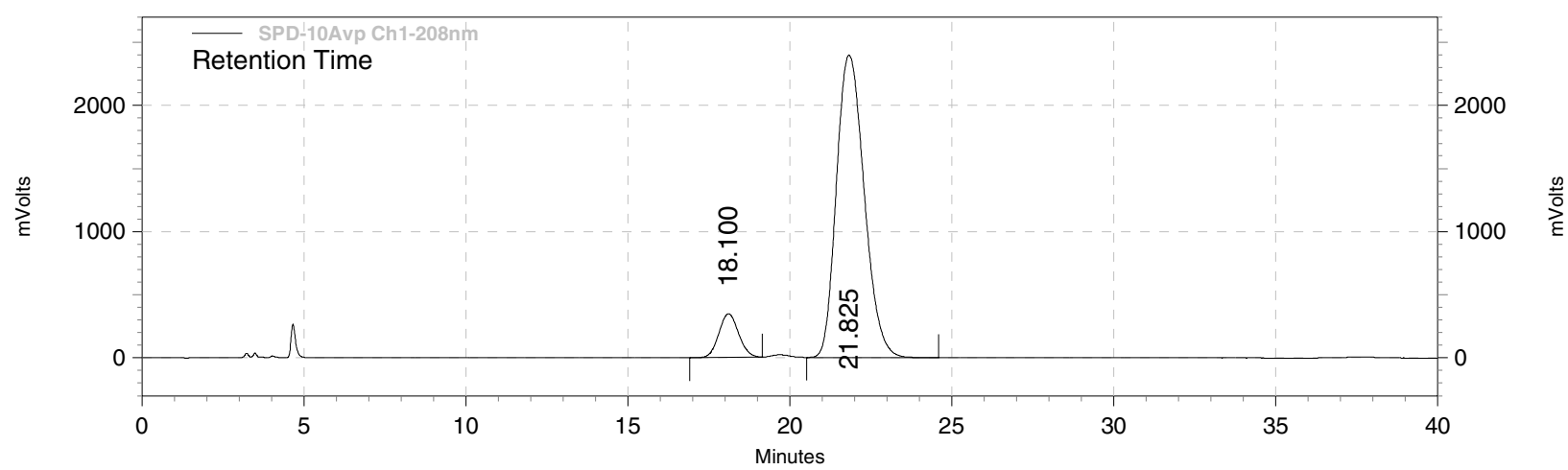

SPD-10Avp Ch1-208nm Results

\begin{tabular}{rrrr|}
\multicolumn{1}{r}{ Retention Time } & Area & Area Percent \\
\hline 18.10 & 14602304 & 9.27 \\
21.82 & 143004701 & 90.73 \\
\hline Totals & & 100.00 \\
\hline
\end{tabular}




\section{\%ee analysis for $5 a$}

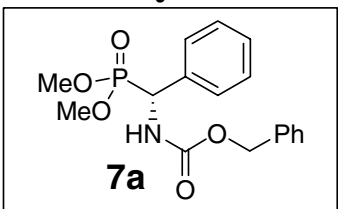

Chiral HPLC: Chiralcel OD, 95:05 Hex:IPA, 1.0 ml/min, 208 nm

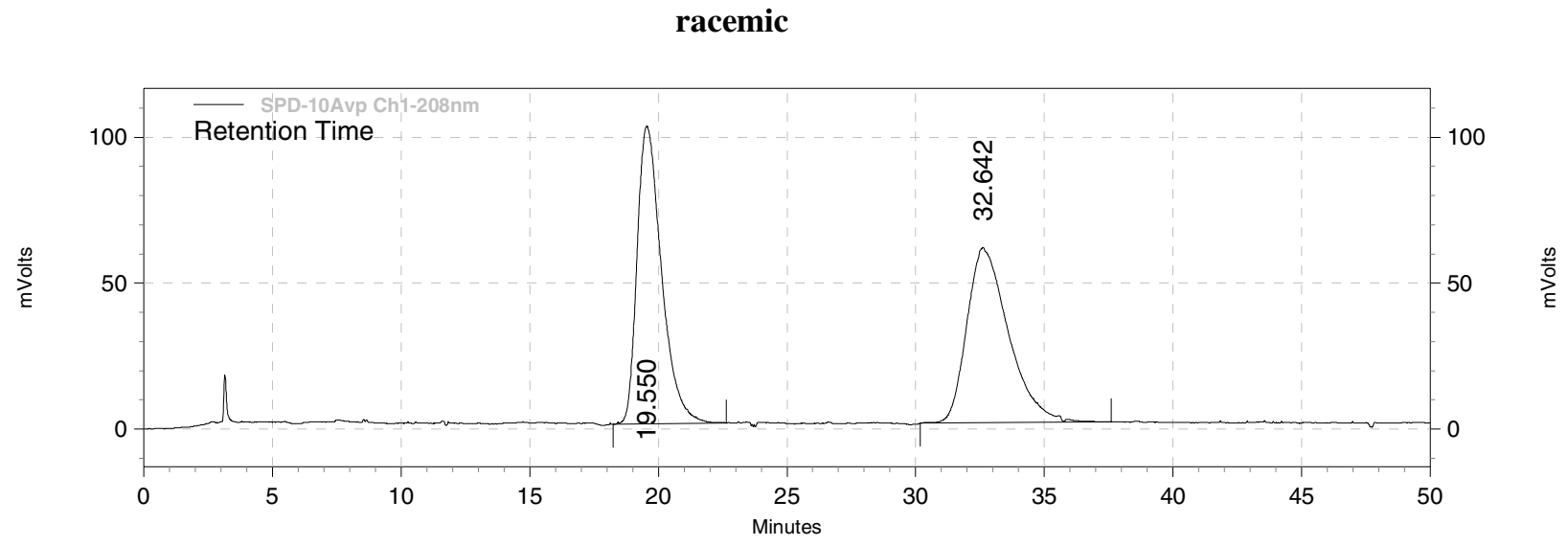

SPD-10Avp Ch1-208nm Results

\begin{tabular}{rrrr|} 
Retention Time & Area & Area Percent \\
\hline 19.55 & 6736672 & 49.97 \\
32.64 & 6744031 & 50.03 \\
\hline Totals & 13480703 & 100.00 \\
\hline
\end{tabular}

$97 \% e e$

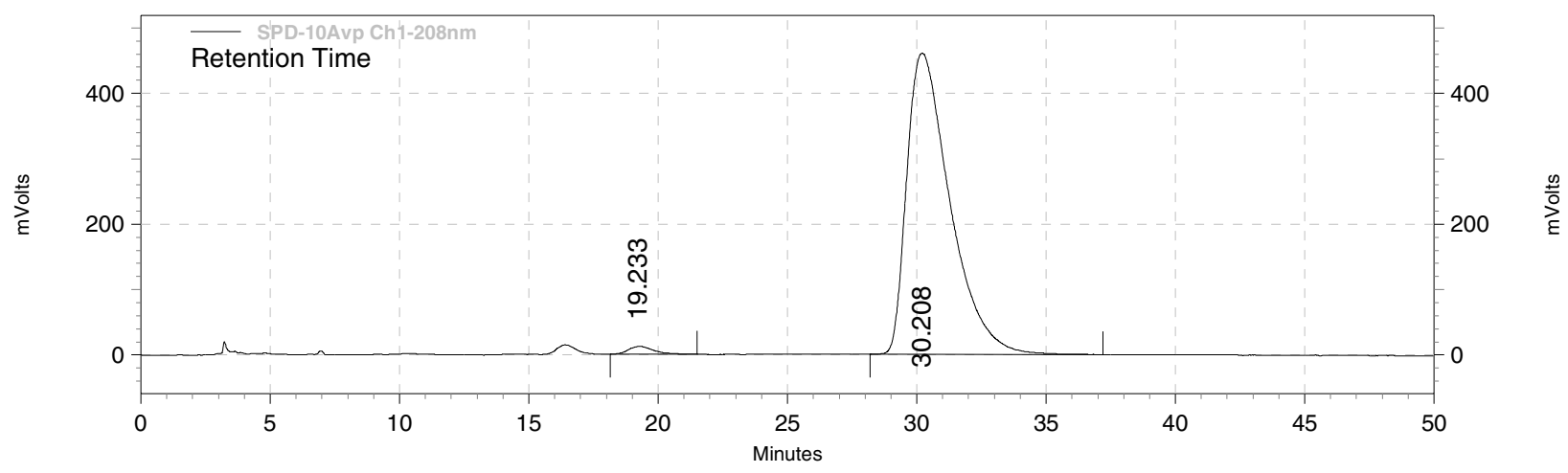

SPD-10Avp Ch1-208nm Results

Retention Time

Area

Area Percent

19.23
30.21

774254

1.43

30.21

53519009

98.57

Totals

54293263

100.00 


\section{\%ee analysis for $\mathbf{5 b}$}

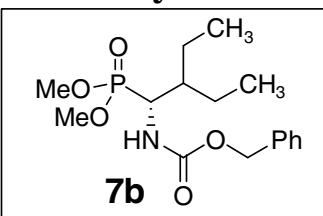

Chiral HPLC: Chiralcel OD, 95:05 Hex:IPA, $1.0 \mathrm{ml} / \mathrm{min}, 208$ nm

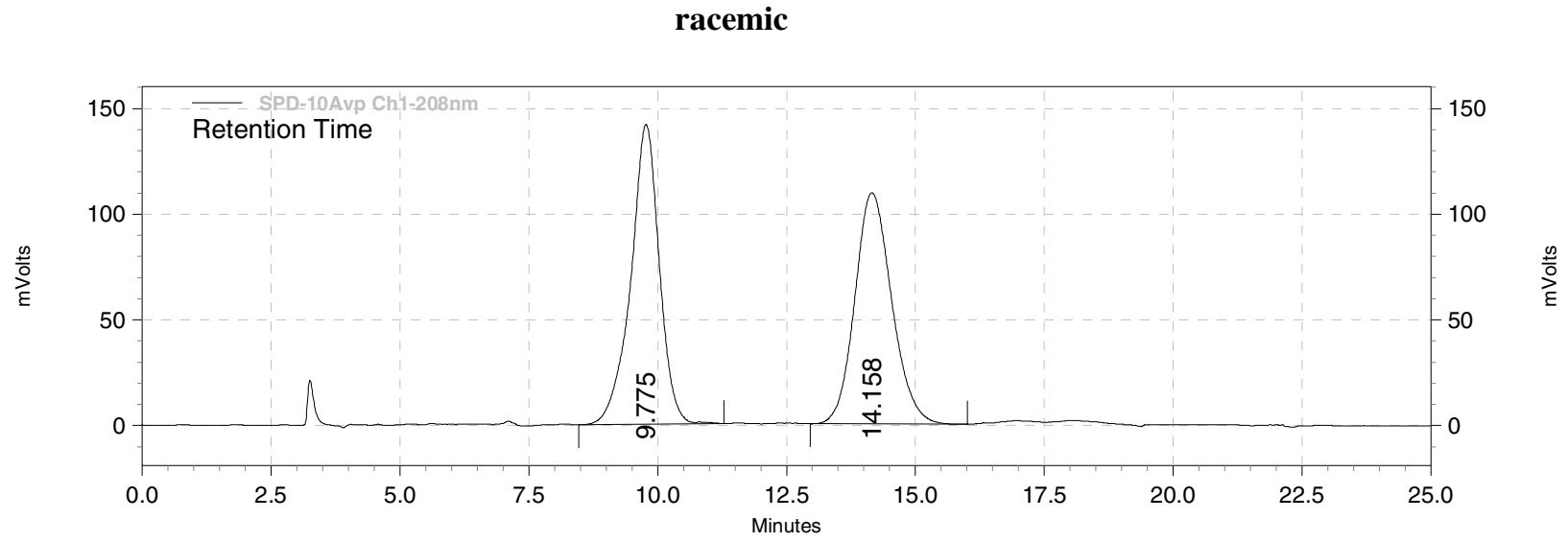

SPD-10Avp Ch1-208nm Results

Retention Time

9.78
14.16

Area

Area Percent

5464457

5444236

50.09

49.91

\begin{tabular}{|r|r|r|}
\hline Totals & 10908693 & 100.00 \\
\hline
\end{tabular}

$96 \% e e$

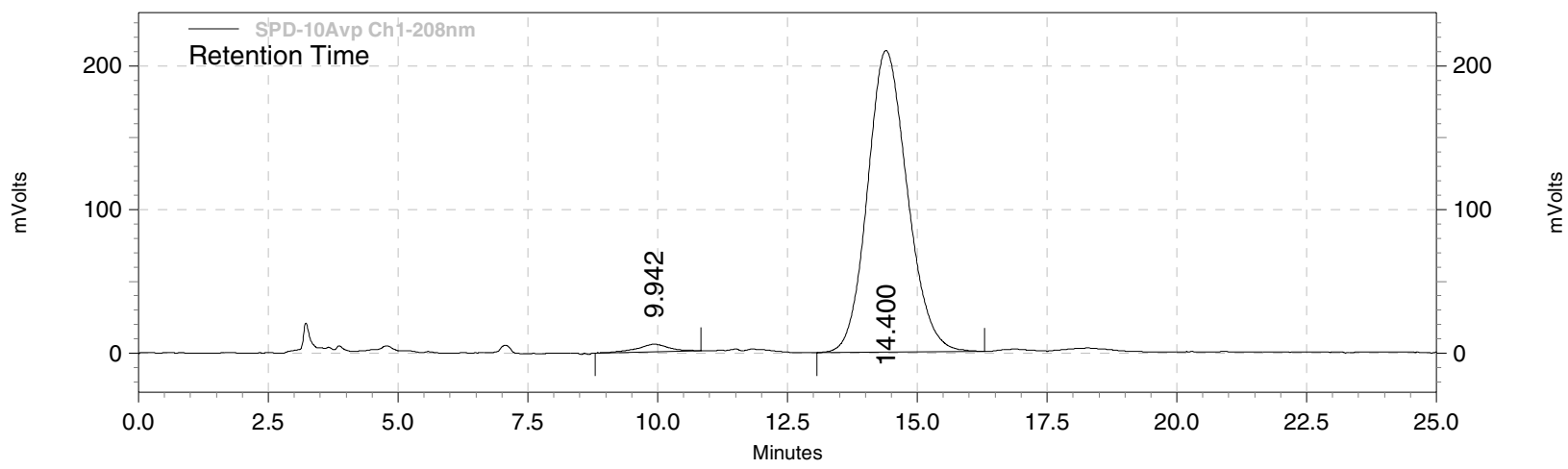

SPD-10Avp Ch1-208nm Results

Retention Time

9.94
14.40

Area

Area Percent

14.40

11179785

1.92

98.08

Totals

11398168

100.00 


\section{\%ee analysis for $5 f$}

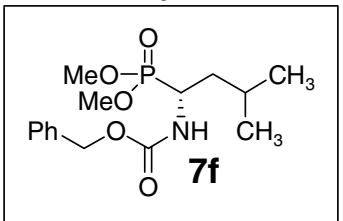

\section{Chiral HPLC: Chiralcel OD, 95:05 Hex:EtOH, $1.0 \mathrm{ml} / \mathrm{min}, 208$ nm}

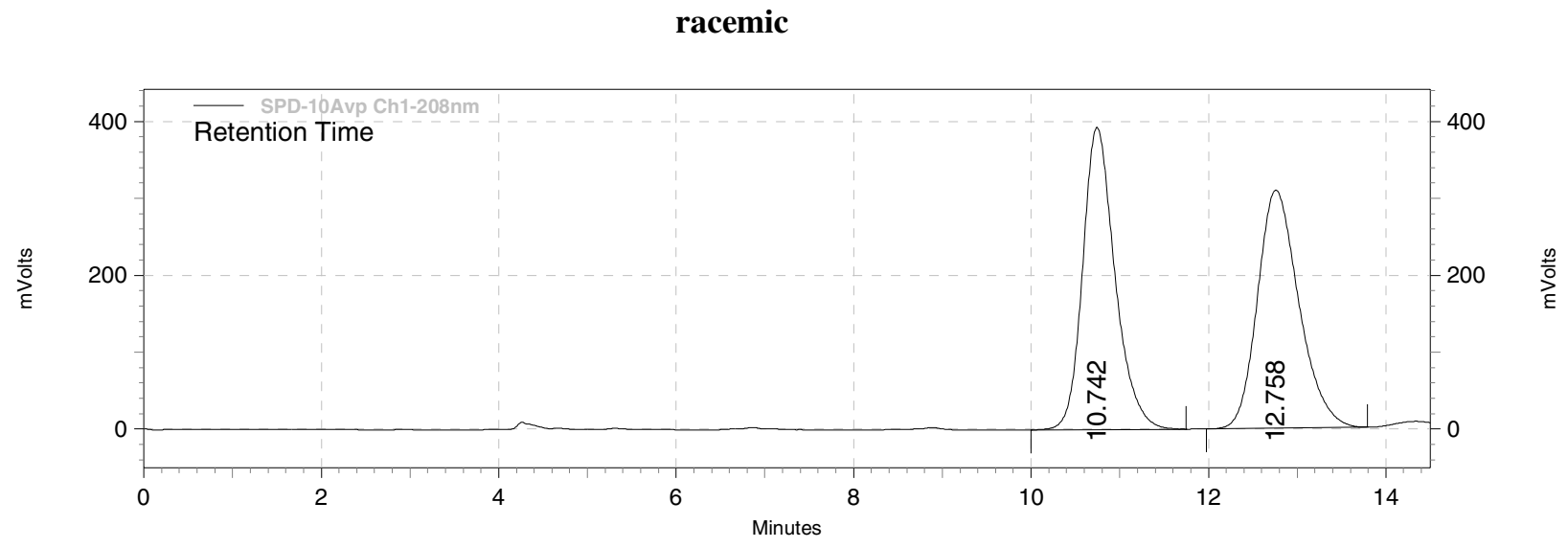

SPD-10Avp Ch1-208nm Results

Retention Time

10.74
12.76

Area

Area Percent

9944761

10031496

49.78

$98 \% e e$

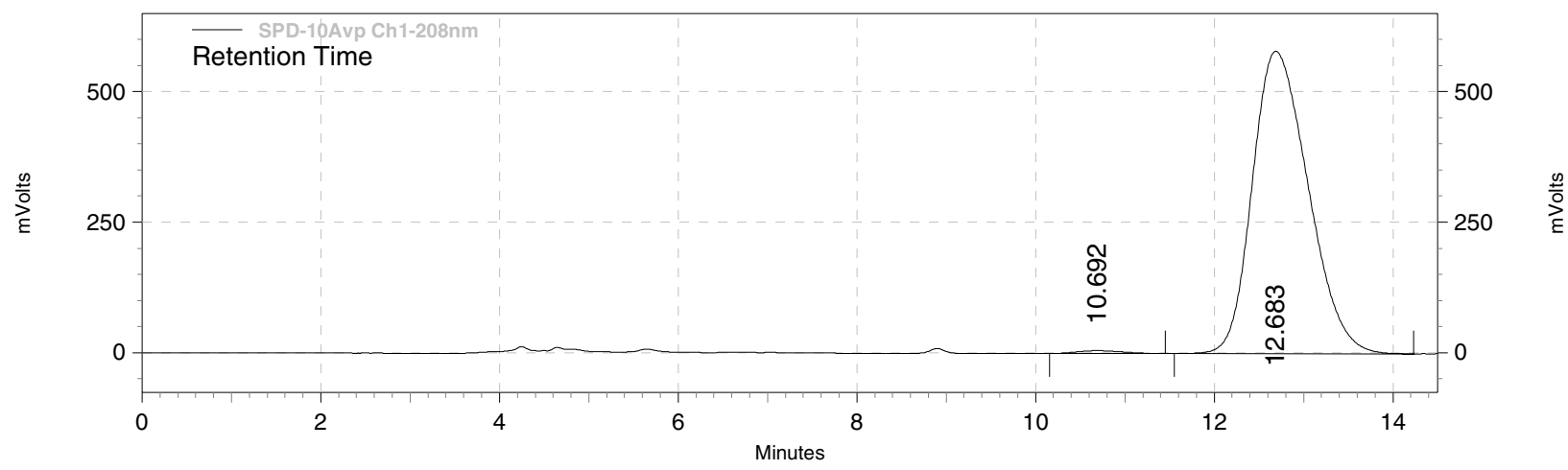

SPD-10Avp Ch1-208nm Results

\begin{tabular}{r|r|r|} 
Retention Time & Area & Area Percent \\
\hline 10.69 & 192377 & 0.76 \\
12.68 & 25149372 & 99.24 \\
\hline Totals & & 100.00 \\
\hline
\end{tabular}

